\title{
SURROGATE-BASED GLOBAL OPTIMIZATION OF COMPOSITE MATERIAL PARTS UNDER DYNAMIC LOADING
}

\author{
A Thesis \\ Submitted to the Faculty \\ of \\ Purdue University \\ by \\ Homero Santiago Valladares Guerra

\begin{abstract}
In Partial Fulfillment of the
Requirements for the Degree

of

Master of Science in Mechanical Engineering
\end{abstract}

August 2017

Purdue University

Indianapolis, Indiana 


\section{THE PURDUE UNIVERSITY GRADUATE SCHOOL STATEMENT OF COMMITTEE APPROVAL}

Dr. Andres Tovar, Chair

Department of Mechanical Engineering

Dr. Alan Jones

Department of Mechanical Engineering

Dr. Sohel Anwar

Department of Mechanical Engineering

Approved by:

Dr. Sohel Anwar

Chair of the Graduate Program 
Dedicated to my family and especially to my parents, Angel and Rosalia, and my siblings Norma, Juan and Angelito. I thank God everyday for their presence in my life. 


\section{ACKNOWLEDGMENTS}

I would like to express my most sincere gratitude to my advisor, Dr. Andres Tovar, for his guidance this two years. Working under his direction in the Engineering Design Research Laboratory has helped me to grow professionally and personally. I have received invaluable lessons from him that I will remember for the rest of my life. I also want to thank the members of my committee, Dr. Sohel Anwar and Dr. Alan Jones for their invaluable suggestions to improve this research project.

I gratefully acknowledge the financial support that I received form the Fulbright Commission of Ecuador and the Department of Mechanical Engineering. Without their support, I could have never had this life-changing experience.

Finally, I want to thank my laboratory fellows, Jennifer Solis-Ocampo, Arturo Garcia, Prathamesh Chaudhari, Prasad Tapkir, Kai Liu and Samuel Attoye, for their

help during this project. A special thanks to Jennifer for her honest friendship and motivation to continue growing in my Catholic faith. 


\section{TABLE OF CONTENTS}

Page

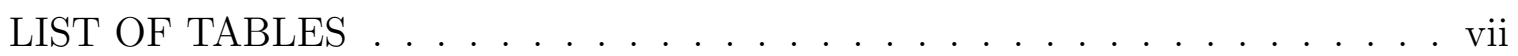
LIST OF FIGURES . . . . . . . . . . . . . . . . . . . viii

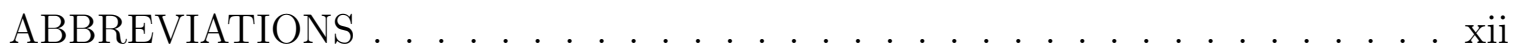

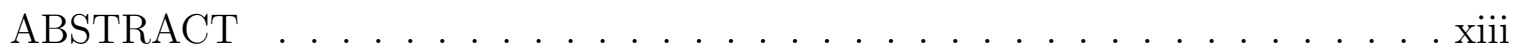

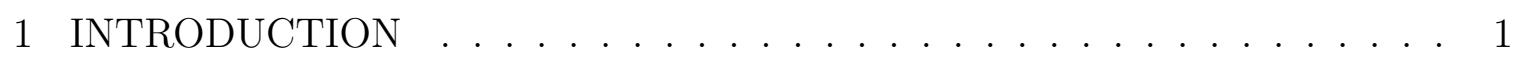

1.1 Composite Materials . . . . . . . . . . . . . . . . . 1

1.2 Design Optimization . . . . . . . . . . . . . . . 2

1.3 Design Optimization of Composites . . . . . . . . . . . . . . . . 4

1.4 Motivation and Objectives . . . . . . . . . . . . 7

1.4.1 Motivation .................. 7

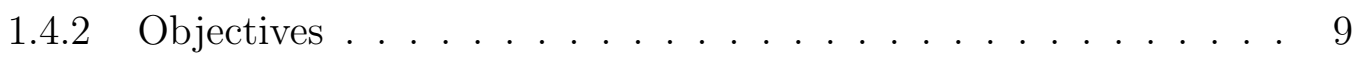

2 FINITE ELEMENT ANALYSIS OF COMPOSITES . . . . . . . . . . . . 10

2.1 Modeling Approach . . . . . . . . . . . . . . . 10

2.1.1 Single-Layer Approach . . . . . . . . . . . . . . . . . 10

2.1.2 Multiple-Layer Approach . . . . . . . . . . . . . . . . 10

2.1.3 Solid Element Approach . . . . . . . . . . . . . . . . . 11

2.1.4 Layered Solid Element Approach . . . . . . . . . . . . . . 11

2.2 Material Models . . . . . . . . . . . . . . . . . . 11

2.2.1 MAT_054/055: Enhanced Composite Damage Model . . . . . . 13

2.3 Basic LS-DYNA Keywords for Composites Analysis . . . . . . . . . . . 17

2.3.1 INTEGRATION_SHELL . . . . . . . . . . . . . . . . 17

2.3.2 SECTION_SHELL . . . . . . . . . . . . . . . . . 18

2.3.3 PART_COMPOSITE ................... 18

2.3.4 ELEMENT_SHELL_COMPOSITE . . . . . . . . . . . . . 19 
2.3.5 CONTROL_ACCURACY .................... 19

2.3.6 CONTROL_SHELL . . . . . . . . . . . . . . . . . . 20

2.3.7 DAMPING_PART_STIFFNESS . . . . . . . . . . . . 20

3 SURROGATE-BASED GLOBAL OPTIMIZATION . . . . . . . . . . . . . 21

3.1 Metamodel Generation . . . . . . . . . . . . . . . . 21

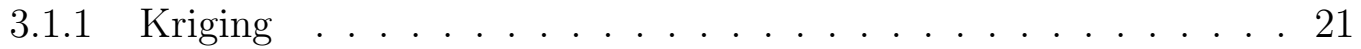

3.1 .2 Latin Hypercube Sampling . . . . . . . . . . . . . . . . . . . . 28

3.1.3 Model Validation . . . . . . . . . . . . . . . . . 29

3.2 Metamodel-Based Global Optimization . . . . . . . . . . . . . 31

3.2 .1 Expected Improvement . . . . . . . . . . . . . . . . . . 31

3.2.2 Multi-Objective Expected Improvement [15] . . . . . . . . . . . 34

3.2.3 The Efficient Global Optimization Algorithm . . . . . . . . . . . 36

3.3 Global Optimization of Composite Materials Parts under Dynamic Loading . . . . . . . . . . . . . . . . . 37

4 NUMERICAL EXAMPLES . . . . . . . . . . . . . . . . . 40

4.1 Minimum Penetration under Dynamic Load on an Armor Plate . . . . 42

4.1 .1 Symmetric Impact . . . . . . . . . . . . . . . . . . . . 43

4.1 .2 Non-Symmetric Impact . . . . . . . . . . . . . . . . . . 50

4.2 Minimum Displacement and Acceleration under Dynamic Load on a

Composite Tube . . . . . . . . . . . . . . . . . 56

4.2.1 Axial Impact . . . . . . . . . . . . . . . . . 57

4.2 .2 Oblique Impact . . . . . . . . . . . . . . . . . . . . . 68

5 SUMMARY AND RECOMMENDATIONS . . . . . . . . . . . . . . . 74

REFERENCES . . . . . . . . . . . . . . . 76 


\section{LIST OF TABLES}

Table

Page

2.1 Composite models in LS-DYNA - Progressive failure degradation law. . . . 12

2.2 Composite models in LS-DYNA - Damage mechanics degradation law. . . 12

2.3 Composite models in LS-DYNA - No failure models. . . . . . . . . . . . . 12

2.4 Keywords options to define a laminate in LS-DYNA. . . . . . . . . . . . . 17

4.1 MAT_054/055 parameters for application examples . . . . . . . . . . . . 41

4.2 MAT_020 parameters for application examples . . . . . . . . . . . . . . . 42

4.3 Kriging surrogate forms . . . . . . . . . . . . . . . . . . . . 42

4.4 LHS sampling - Armor problem . . . . . . . . . . . . . . . . . 44

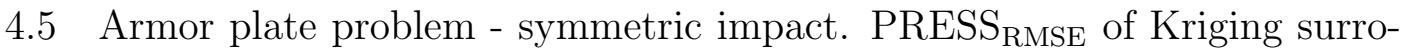
gates using different regression and correlation functions. . . . . . . . . . . 45

4.6 Optimal designs armor problem, symmetric impact. . . . . . . . . . . 45

4.7 Armor plate problem - non-symmetric impact. PRESS RMSE $_{\text {of Kriging }}$ surrogates using different regression and correlation functions. . . . . . . . 50

4.8 LHS sampling - Composite tube problem . . . . . . . . . . . . . . 58

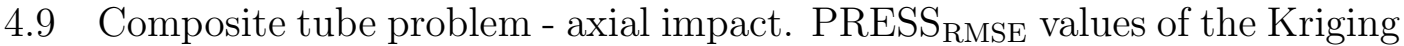
metamodels for the wall displacement. . . . . . . . . . . . . . . 58

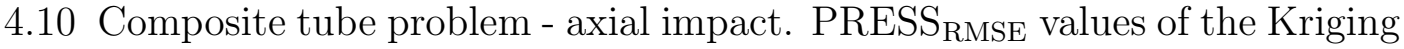
meatmodels for the peak acceleration of the wall. . . . . . . . . . . . 59

4.11 Composite tube problem - axial impact. Optimal designs and responses . . 66

4.12 Composite tube problem - axial impact. Optimal feasible designs . . . . . 67

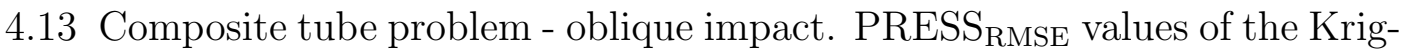
ing metamodels for the wall displacement. . . . . . . . . . . . . . . 68

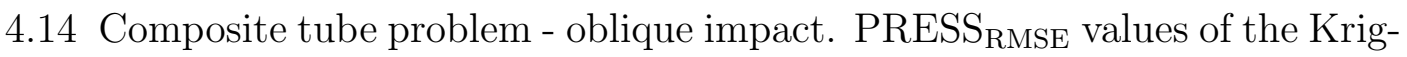
ing metamodels for the peak acceleration of the wall. . . . . . . . . . 69

4.15 Composite tube problem - oblique impact. Optimal feasible designs . . . . 72 


\section{LIST OF FIGURES}

Figure

1.1 Multi-objective optimization problem. Design domain and objective domain. Feasible designs (green dots) and non-feasible designs (blue dots). Pareto front (black dots). . . . . . . . . . . . . . . . . 4

1.2 Multi-objective optimization problem. Definition of the Pareto front (black), dominated designs (green) and utopia point. . . . . . . . . . . . 5

1.3 The solid line represents an objective function that has been sampled at the 4 gray dots. The dashed line is a Kriging prediction fit to those points. False optimum (green dot) and true optimum (red dot). . . . . . . . . 8

1.4 Kriging surrogate and its standard error for a four-point data set. . . . . . 8

2.1 MAT_054/055 input card example . . . . . . . . . . . . . . . . 14

2.2 Example of SECTION_SHELL keyword. . . . . . . . . . . . . . 18

2.3 Example of PART_COMPOSITE keyword. . . . . . . . . . . . . . . . . . . 19

2.4 Effect of CONTROL_ACCURACY over the material coordinates of deformed shell elements. . . . . . . . . . . . . . . . . . . 20

3.1 Kriging predictions and uncertainties. . . . . . . . . . . . . 24

3.2 Illustration of how correlation affects Kriging prediction. The prediction of $\mathrm{x}^{*}$, that is close to the sample point $\mathrm{x}^{(2)}$, should be adjusted from the regression line to take into account the positive residual at $\mathrm{x}^{(2)}$. . . . . 25

3.3 Effect of $p_{i}$ in the correlation function. . . . . . . . . . . . . . 26

3.4 Effect of $\theta_{i}$ in the correlation function. . . . . . . . . . . . . . 27

3.5 LHS with $\mathrm{P}=5$. High correlation sampling and low correlation sampling due to correlation minimization method. . . . . . . . . . . . . . . . . 29

3.6 Cross-validation at the second sampled point. . . . . . . . . . . . . 30

3.7 Expected improvement function with nine samples. The left scale is for the objective function and the right scale for the expected improvement. . 32

3.8 Progress of the optimization process when the designs that maximize the EI are sampled. . . . . . . . . . . . . . . . 33 
Figure

3.9 Multi-objective expected improvement. Centroid of the probability integral (red dot), Pareto front (blue dots) and predicted responses of sample $x$ (green dot) . . . . . . . . . . . . . . . . . . 35

3.10 Efficient global optimization algorithm. . . . . . . . . . . . . . . . 37

3.11 Efficient global optimization algorithm MATLAB - LS-DYNA. . . . . . . . 38

4.1 Armor plate problem - symmetric impact . . . . . . . . . . . . . . . 43

4.2 Armor problem - symmetric impact. Initial Kriging surrogate of the armor's penetration based on the 21 LHS sampled points shown as dots. . . 44

4.3 EGO optimization progress - Armor problem, symmetric impact. Current sample (grey), best current design (purple), last sampled design (cyan). Iterations 1,2 and $3 . \ldots \ldots \ldots 6$

4.4 EGO optimization progress - Armor problem, symmetric impact. Current sample (grey), best current design (purple), last sampled design (cyan). Iterations 4,5 and $6 \ldots \ldots \ldots$. . . . . . . . . . . . . . . . . . . . . .

4.5 Armor plate problem - symmetric impact. Expected improvement at each iteration. . . . . . . . . . . . . . . . . . . 48

4.6 Armor plate problem - symmetric impact. Response of the designs sampled by EGO at each iteration. Optimal responses (gray dots). . . . . . . . 48

4.7 EGO optimization progress - Armor problem, symmetric impact. Current sample (grey), best current design (purple), last sampled design (cyan).

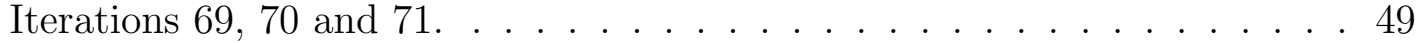

4.8 Armor plate problem - non-symmetric impact . . . . . . . . . . . . . 50

4.9 Armor problem, non-symmetric impact. Initial Kriging surrogate of the armor's penetration based on the 21 LHS sampled points shown as dots. . 51

4.10 EGO optimization progress - Armor problem, non-symmetric impact. Current sample (grey), best current design (purple), last sampled design (cyan). Iterations 1,2 and $3 . \ldots \ldots \ldots$. . . . . . . . . . . . . . . . . . . .

4.11 EGO optimization progress - Armor problem, non-symmetric impact. Current sample (grey), best current design (purple), last sampled design (cyan). Iterations 4,5 and $6 . \ldots \ldots \ldots$

4.12 Armor plate problem - non-symmetric impact. Expected improvement at each iteration. . . . . . . . . . . . . . . . . . . 54

4.13 Armor plate problem - non-symmetric impact. Response of the designs sampled by EGO at each iteration. Optimal response (gray dot). . . . . . 54 
Figure $\quad$ Page

4.14 EGO optimization progress - Armor problem, non-symmetric impact. Current sample (grey), best current design (purple), last sampled design (cyan). Iterations 102, 103 and 104 . . . . . . . . . . . . . . . . . 55

4.15 Composite tube problem - axial impact. . . . . . . . . . . . . . . 57

4.16 Composite tube problem. Sampling over design space using 43 LHS designs. 59

4.17 Composite tube problem - axial impact. Initial responses for the 43 LHS samples. LHS samples (grey), Pareto front (purple). . . . . . . . . . . 60

4.18 EGO optimization progress - Composite tube problem, axial impact. Dominated designs (grey), Pareto front (purple), last sampled design (cyan). Iterations $1,2,3$ and $4 \ldots \ldots \ldots 1$

4.19 Composite tube problem - axial impact. Responses at the last iteration. Dominated designs (grey), Pareto front (purple) . . . . . . . . . . . 62

4.20 Composite tube problem - axial impact. Initial and final Pareto fronts. . . 62

4.21 Composite tube problem - axial impact. Expected improvement at each iteration. . . . . . . . . . . . . . . . . 63

4.22 Composite tube problem - axial impact. Non-feasible designs in the final Pareto front. . . . . . . . . . . . . . . . . . . . 63

4.23 Composite tube problem - axial impact. Responses at the last iteration using constrained improvement formulation. . . . . . . . . . . . . . . 65

4.24 Composite tube problem - axial impact. Comparison of Pareto fronts using constrained and unconstrained expected improvement. . . . . . . . 65

4.25 Composite tube problem - oblique impact. . . . . . . . . . . . . . . 68

4.26 Composite tube problem - oblique impact. Initial responses using the 43 LHS samples. LHS samples (grey), Pareto front (purple). . . . . . . . . . . 69

4.27 EGO optimization progress - Composite tube problem, oblique impact. Dominated designs (grey), Pareto front (purple), last sampled design (cyan). Iterations $1,2,3$ and $4 \ldots \ldots \ldots$. . . . . . . . . . . . 70

4.28 Composite tube problem - oblique impact. Non-feasible designs in the final Pareto front. . . . . . . . . . . . . . . . . . . . . . 71

4.29 Composite tube problem - oblique impact. Responses at the last iteration using constrained improvement formulation. . . . . . . . . . . . . . . 71

4.30 Composite tube problem - oblique impact. Comparison of Pareto fronts using constrained and unconstrained expected improvement. . . . . . . . 73 
4.31 Composite tube problem - oblique impact. Expected improvement at each iteration. . . . . . . . . . . . . . . . . 73 


\section{ABBREVIATIONS}

MMCs: Metal Matrix Composites

PMCs: Polymer Matrix Composites

FRP: $\quad$ Fiber Reinforced Polymer

CFRP: Carbon Fiber Reinforced Polymers

MFD: $\quad$ Method of Feasible Directions

SQP: $\quad$ Sequential Quadratic Programming

GA: Genetic Algorithms

PSO: Particle Swarm Optimization

RBF: Radial Basis Functions

RSM: Response Surface Methodology

MARS: Multivariate Adaptive Regression Splines

EI: $\quad$ Expected Improvement

CEI: Constrained Expected Improvement

MEI: Multi-objective Expected Improvement

EGO: Efficient Global Optimization

FEA: $\quad$ Finite Element Analysis

FE: $\quad$ Finite Element

MLE: Maximum Likelihood Estimation

CLF: Concentrated Likelihood Function

CDF: Cumulative Density Function

PDF: Probability Density Function

regpoly0: Zero Order Regression Function

regpoly1: First Order Regression Function

regpoly2: Second Order Regression Function 


\begin{abstract}
Valladares Guerra, Homero Santiago M.S.M.E., Purdue University, August 2017. Surrogate-Based Global Optimization of Composite Material Parts under Dynamic Loading. Major Professor: Andres Tovar.
\end{abstract}

The design optimization of laminated composite structures is of relevance in automobile, naval, aerospace, construction and energy industry. While several optimization methods have been applied in the design of laminated composites, the majority of those methods are only applicable to linear or simplified nonlinear models that are unable to capture multi-body contact. Furthermore, approaches that consider composite failure still remain scarce. This work presents an optimization approach based on design and analysis of computer experiments (DACE) in which smart sampling and continuous metamodel enhancement drive the design process towards a global optimum. Kriging metamodel is used in the optimization algorithm. This metamodel enables the definition of an expected improvement function that is maximized at each iteration in order to locate new designs to update the metamodel and find optimal designs. This work uses explicit finite element analysis to study the crash behavior of composite parts that is available in the commercial code LS-DYNA. The optimization algorithm is implemented in MATLAB. Single and multi-objective optimization problems are solved in this work. The design variables considered in the optimization include the orientation of the plies as well as the size of zones that control the collapse of the composite parts. For the ease of manufacturing, the fiber orientation is defined as a discrete variable. Objective functions such as penetration, maximum displacement and maximum acceleration are defined in the optimization problems. Constraints are included in the optimization problem to guarantee the feasibility of the solutions provided by the optimization algorithm. The results of this study show 
that despite the brittle behavior of composite parts, they can be optimized to resist and absorb impact. In the case of single objective problems, the algorithm is able to find the global solution. When working with multi-objective problems, an enhanced Pareto is provided by the algorithm. 


\section{INTRODUCTION}

\subsection{Composite Materials}

Composites material structures are widely used in Aerospace, Automobile, Construction and Energy industries. They provide benefits such as weight reduction, durability, high strength and energy absorption. According to the United States Department of Energy, the transportation sector consumes almost $30 \%$ of the U.S. energy. It has been estimated that $75 \%$ of vehicles fuel consumption is related to its weight [1]. Composite materials have made possible to achieve weight reductions but maintaining high structural performance of mechanical parts. Composites are anisotropic materials that combine two or more materials to obtain better properties than the individual components that are combined in a macroscopic scale. There are different types of composites in nature. Depending on their processing routes, they are classified as natural composites, Bio-composites, Carbon-carbon composites, Ceramic Matrix Composites (CMCs), Metal Matrix Composites (MMCs) and Polymer Matrix Composites (PMCs). PMCs are the most used in industry. They cover $75 \%$ of the world composite market [1]. Composites can be also classified by filler types as particle-reinforced, fiber-reinforced and structural.

Fiber Reinforced Polymer (FRP) composites consist of fiber reinforcements and a polymeric matrix. The type and distribution of the fiber reinforcement determine the stiffness and strength. Fibers are commonly made of materials with high strength and elasticity modulus, e.g., Carbon Fiber Reinforced Polymers (CFRP). The purpose of the matrix is to hold the fibers together, to transfer the external loads to the fibers and to protect the composite from external damage [1].

In industry, composite parts are commonly made of laminates which is conformed by a stack of layers (plies). Each layer is composed of a light matrix and high strength 
fiber reinforcements. The performance of a composite structure depends on many factors such as matrix and fiber materials, ply thickness, fiber orientation and structural shape.

\subsection{Design Optimization}

The performance of most systems in engineering depends on several parameters (design variables). Design optimization techniques assist engineers to find the parameters that lead to the optimal system performance. The optimization process often involves trade-off analysis due to the presence of competing objective functions, i.e., improving one objective implies the worsening of others. In the formulation of the optimization problem, realistic boundaries (constraints) are defined in order to prevent impractical solutions [2]. A multi-objective optimization problem is defined as,

$$
\begin{array}{ll}
\text { find } & \mathbf{x} \in \mathbb{R}^{n_{d v}} \\
\text { minimize } & \mathbf{f}(\mathbf{x}) \\
\text { subject to } & g_{i}(\mathbf{x}) \leq 0, i=1, \ldots, n_{c} \\
& \mathbf{x}_{\text {low }} \leq \mathbf{x} \leq \mathbf{x}_{u p}
\end{array}
$$

where $\mathbf{x}=\left[x_{1}, \ldots, x_{n_{d v}}\right]$ is the design vector, $n_{d v}$ is the number of design variables (design space dimension), $\mathbf{f}(\mathbf{x})=\left[f_{1}(\mathbf{x}), \ldots, f_{k}(\mathbf{x})\right]$ is the vector of objective functions, $k$ is the number of objective functions (response space dimension), $g_{i}(\mathbf{x})$ are the problem constraints, $n_{c}$ is the number of constraints, and $\mathbf{x}_{\text {low }} \leq \mathbf{x} \leq \mathbf{x}_{u p}$ are the lower and upper bounds of the design variables.

The following example is provided to illustrate the components of a multi-objective optimization problem. It corresponds to the optimization of a beam in cantiliever. The purpose of the problem is to determine the values of $x_{1}$ and $x_{2}$ (beam dimensions) in order to minimize the beam deflection, $f_{1}\left(x_{1}, x_{2}\right)$, and the beam mass, $f_{2}\left(x_{1}, x_{2}\right)$. 
The stress constraint $g\left(x_{1}, x_{2}\right)$ prevents material failure. For details about the problem formulation, see $[2]$.

$$
\begin{array}{ll}
\text { find } & \mathbf{x}=\left[x_{1} x_{2}\right] \in \mathbb{R}^{2} \\
\text { minimize } & {\left[\begin{array}{l}
f_{1}\left(x_{1}, x_{2}\right) \\
f_{2}\left(x_{1}, x_{2}\right)
\end{array}\right]} \\
\text { subject to } & g\left(x_{1}, x_{2}\right)=\sigma\left(x_{1}, x_{2}\right)-\frac{s_{y}}{n} \leq 0 \\
& 0.001 \leq x_{1} \leq 0.750(\mathrm{~m}) \\
& 0.001 \leq x_{2} \leq 0.075(\mathrm{~m})
\end{array}
$$

An optimization problem involves a design space and an objective space as illustrated in Fig. 1.1. Each member of the design space is mapped into the objective space. The design space is limited by the lower and upper bounds of the design variables. In the design space, there are designs that satisfy the problem constraints (feasible designs) and designs that do not (non-feasible designs). The Pareto front is the set of best compromised solutions, i.e., no further improvement can be achieved in one function without affecting the others [2].

Fig. 1.2 provides a detailed explanation of the Pareto front. If designs $\mathrm{A}$ and $\mathrm{B}$ are compared, both designs have the same mass; however, design $\mathrm{B}$ has a larger deflection than A. A is a better design than B since by the same mass it presents lower deflection. Following the same analysis, $\mathrm{C}$ is a better design than $\mathrm{B}$ since it presents the same deflection but less mass. Finally, if designs A and C are compared, neither is dominated by the other since each one is better in one objective function. The Pareto front, also known as the Pareto optimal set, is the set of solutions that are better in at least one objective function.

Solutions that are not part of the Pareto set are called dominated solutions. The solution of a multi-objective optimization problem is not unique. The designer task is to select designs from the Pareto optimal. The utopia or ideal point can be used for the selection process. The utopia point has all the minimum function values, $f_{1 \min }\left(x_{1}, x_{2}\right)$ and $f_{2 \min }\left(x_{1}, x_{2}\right)$, as coordinates. If possible, the utopia point repre- 

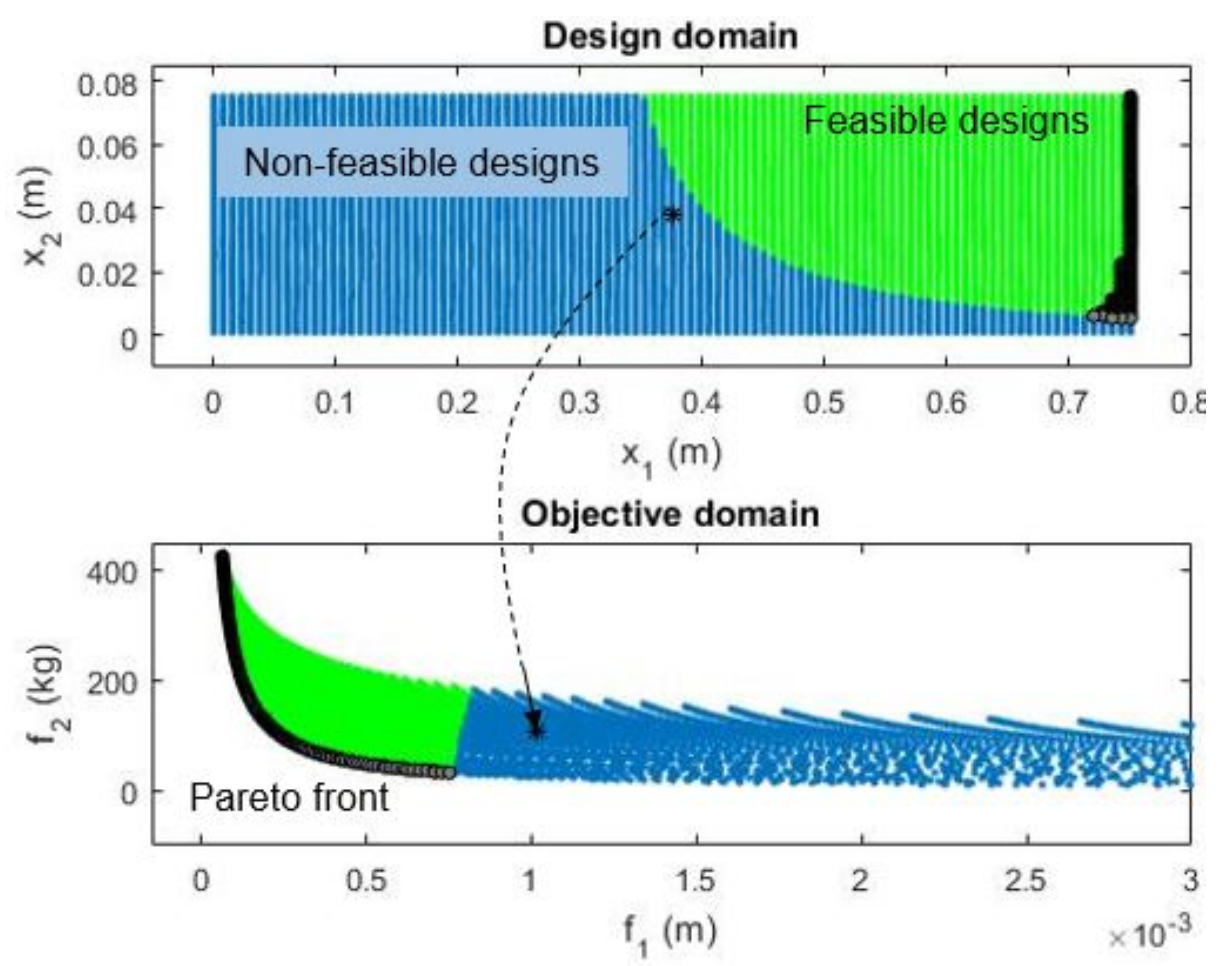

Figure 1.1. Multi-objective optimization problem. Design domain and objective domain. Feasible designs (green dots) and non-feasible designs (blue dots). Pareto front (black dots).

sents the best performance that the system can achieve given the problem conditions. A practical rule is to choose the Pareto design that is closer to the utopia point [2].

\subsection{Design Optimization of Composites}

The purpose of applying optimization techniques in the design of composite parts is to find the variables that lead to the best performance. Matrix and fiber materials, fiber orientation, ply thickness and geometrical dimensions are common design variables for composites [3]. Objectives for the optimization of composites are the minimization of weight, volume, deflection and crushing forces, or the maximization of buckling loads and specific energy absorption. Realistic constraints are included in the optimization problem to satisfy manufacturing requirements, e.g., maximum number of plies or a discrete range for the fiber orientations. The most common opti- 


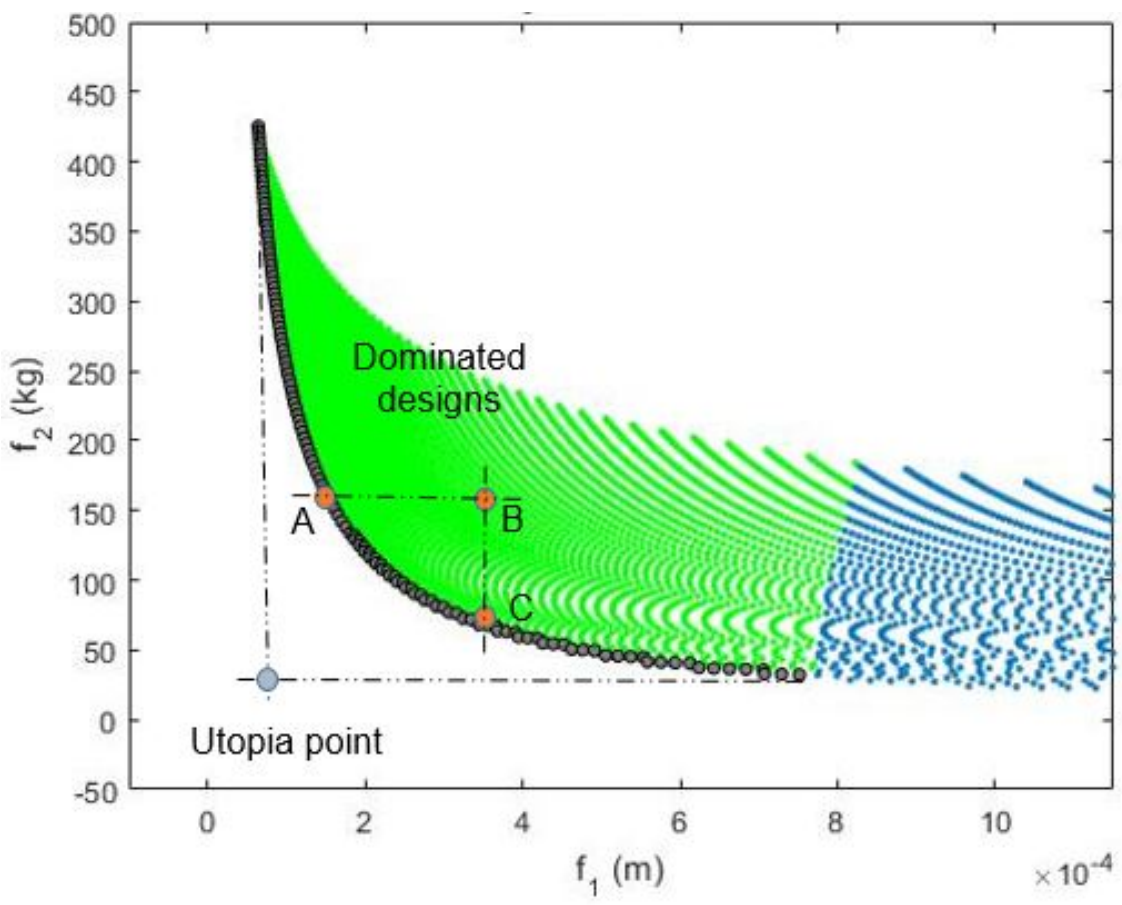

Figure 1.2. Multi-objective optimization problem. Definition of the Pareto front (black), dominated designs (green) and utopia point.

mization techniques that have been used to optimize composites are gradient based, population-based and surrogate-based methods.

Gradient-based optimization algorithms: Gradient-based optimization techniques provide fast convergence when reliable sensitivity coefficients are available [4]. They are not suitable when working with noisy functions and discrete variables. The Method of Feasible Directions (MFD) and Sequential Quadratic Programming (SQP) are examples of gradient based techniques that have been successfully implemented in the design optimization of composites. MFD was used to determine materials and fiber orientations of a wind turbine blade. The objective of the study was to maximize the compressive load that the part can support [5]. SQP was applied in the strength maximization of a composite box-beam structure [3]. Due to the local nature of gradient-based techniques, appropriate starting designs are needed to perform the optimization process.

Population-based optimization algorithms: Although the use of gradientbased techniques is attractive because of their computational convenience, they have 
limitations for the design of composite parts. Generally, the design optimization of composites is a non-convex, multimodal optimization problem that involves continuous and discrete variables [6]. In such cases, the use of population-based algorithms is preferred since they use the responses of several designs in each iteration of the algorithm to find the optimal solution instead of gradient information. Genetic Algorithms (GA) and Particle Swarm Optimization (PSO) are examples of populationbased techniques that are used in composites optimization $[7,8]$. They have been able to find global solutions even under complex loading conditions [9].

\section{Surrogate-based optimization}

It has been proved that population-based methods have high robustness and capability to find global solutions; however, they require a large number of function evaluations to solve the optimization problem [10]. This is a huge limitation when dealing with expensive models such as the crash simulations of composite parts. Those simulations are expensive because of the multi-body contacts and the several failure mechanism of the material. In crash conditions, composites undergo diverse failure mechanisms like matrix cracking and splitting, interlaminar damage (delamination), fiber fracture, frond formation and bending, and friction $[11,12]$.

When dealing with expensive functions, the use of surrogate-based techniques is preferred. A surrogate model, also known as a metamodel, is a fast mathematical approximation to an expensive model. It is created by using the responses of some samples of the design space. Examples of common metamodels for impact analysis are response surface methodology (RSM), radial basis functions (RBF) and Kriging surrogates. Crashworthiness optimization of a full-scale vehicle under frontal collision was succesfully performed using RSM and RBF. The energy absorbed by the vehicle was increased by $25 \%$ compared to the original design. It was also possible to reduce the peak crushing acceleration by $21 \%$ relative to the initial design [13]. By combining Kriging surrogates and GA, the occupant restraint system of a vehicle under frontal impact was optmized. The passenger injury levels were reduced by $36 \%$ in comparison with the baseline design [14]. 


\subsection{Motivation and Objectives}

\subsubsection{Motivation}

Metamodels are excellent options when performing optimization with models that require long-running times. The basic idea of surrogate-based optimization is simple. However, it is important to have special care in details such as the selection scheme of sample points, the approximation method creating the metamodel, and the updating scheme for the metamodel [15].

The simplest way to perform metamodel-based optimization is to build a surrogate model and apply an optimization algorithm to find its minimum at each iteration, then sample the design and use it to update the metamodel iteratively until convergence is reached. The main disadvantage of that searching procedure is that a local or false minimum may be found instead of a global minimum as illustrated in Fig. 1.3. The possibility of finding a local optimum arises because too much emphasis is placed on the exploitation of the metamodel without acknowledging its uncertainties [16].

As illustrated in Fig. 1.4, the metamodel is not accurate along the whole design space. To take uncertainties into account, the metamodel can be updated with samples in areas where large errors are expected. However, while that optimization scheme will explore the design space, it is not truly directed to find the global optimum. An efficient global optimization algorithm balances between metamodel exploitation and design space exploration. [16].

Among the mathematical models available for surrogate-based optimization, Kriging metamodel provides the possibility of quantifying expected prediction errors along the whole design space as shown in Fig. 1.4. This enables the definition of a Expected Improvement (EI) function to direct the optimization process. The use of the EI function balances metamodel exploitation and design space exploration because the function takes contributions from the best current designs in the metamodel and the uncertainties along the design space to suggest new searching regions. 


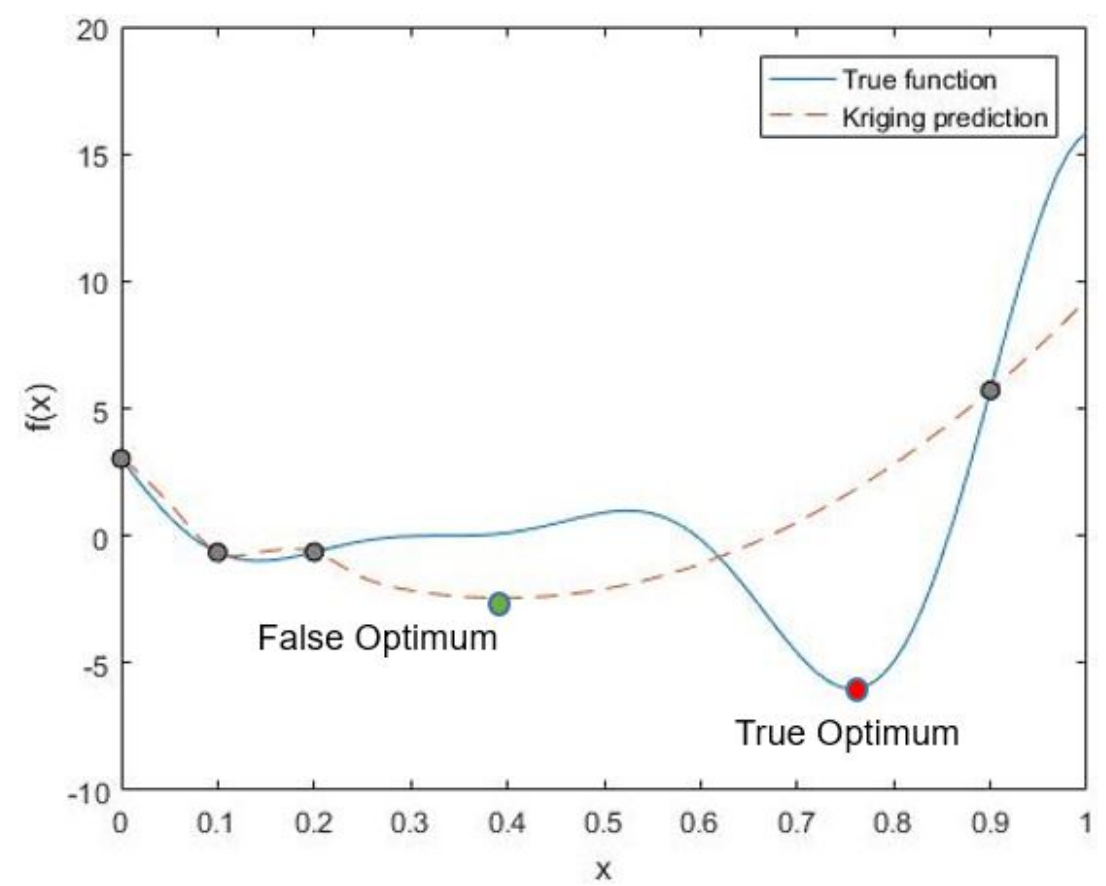

Figure 1.3. The solid line represents an objective function that has been sampled at the 4 gray dots. The dashed line is a Kriging prediction fit to those points. False optimum (green dot) and true optimum (red dot).

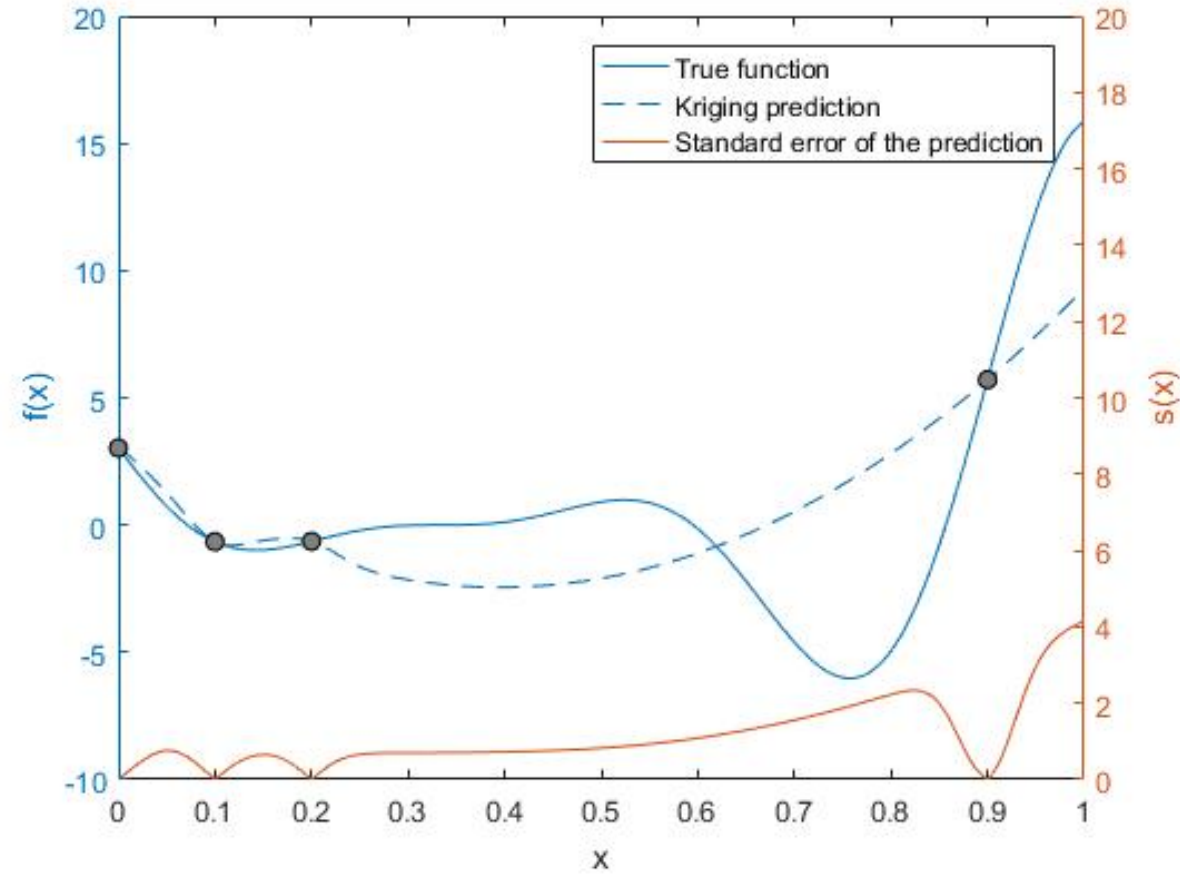

Figure 1.4. Kriging surrogate and its standard error for a four-point data set. 


\subsubsection{Objectives}

Although the design optimization of composite parts using surrogate models have been developed by several authors, most of them only use the predictions of the surrogates to direct the search of the global optimum without acknowledging the metamodel uncertainties. The Efficient Global Optimization (EGO) algorithm proposed by Jones et al. [16] has demonstrated that high accuracy solutions and efficient search processes can be achieved by including metamodel uncertainties in the optimization process. This investigation is devoted to performing the design optimization of fiber-reinforced polymer matrix composites under dynamic loading using the EGO algorithm.

The optimization approach is based on design and analysis of computer experiments (DACE) in which smart sampling and continuous metamodel enhancement drive the design process towards the global optimum. The impact simulations of the composite parts are performed in the general-purpose finite element analysis software LS-DYNA. MATLAB is used to generate the sample designs, execute the LS-DYNA simulations, extract results from the LS-DYNA output files and analysis of the data.

The results of this investigation are described as follows:

Chapter 2 contains the description of the composite material model used to perform the Finite Element Analysis (FEA) simulations in LS-DYNA. Explanations of the keywords for composites simulations are also included.

Chapter 3 provides the principles of Kriging metamodel, EI function, Latin hypercube sampling and validation of surrogates. At the end of the chapter, the implementation of the EGO algorithm is included.

Chapter 4 contains numerical examples that illustrate the use of EGO in the design optimization of composite parts.

Chapter 5 concludes this work with a summary of the investigation and final recommendations. 


\section{FINITE ELEMENT ANALYSIS OF COMPOSITES}

During the last two decades, the capability of Finite Element (FE) models to simulate complex systems has significantly increased. Nowadays, it is possible to evaluate dynamic models with contacts between multiple parts and nonlinearities in the material and geometry of the parts. Explicit finite element formulations are used to solve these models. Among the different commercial codes that have implemented explicit finite element analysis formulation, LS-DYNA is the most popular for crash analysis. Composite parts and be modeled using shell elements, solid elements and layered solid elements to create detailed models and large-scale models. Detailed models capture the failure mechanisms within the material while large-scale models place more emphasis in the structural behavior of the composite parts [17].

\subsection{Modeling Approach}

\subsubsection{Single-Layer Approach}

In the single-layer approach, the composite laminate is modeled with one layer of shell elements and an integration rule that is defined through the shell thickness. Each integration point represents a ply of the laminate. The single-layer approach is popular when dealing with large-scale structural problems due to its low computational cost. The single-layer approach is adopted in this work to perform the structural optimization of the composite parts.

\subsubsection{Multiple-Layer Approach}

In the multiple-layer approach, the laminate is modeled using multiple layers of shell elements. The layers are stacked together using contact definitions between them 
[18]. In LS-DYNA, delamination is studied using CONTACT_AUTOMATIC_TO_ SURFACE_TIEBREAK. The multiple-layer approach is more computationally expensive than the single-layer approach but less costly than three-dimensional models.

\subsubsection{Solid Element Approach}

In thick composite structures, plane stress condition is not applicable. The stress distribution through the thickness of the laminate is not negligible. Each ply of the laminate can be represented using solid elements to account for stress distribution effects. It is a costly and cumbersome solution when dealing with large structures [19].

\subsubsection{Layered Solid Element Approach}

In the layered solid element approach, the composite parts are modeled using a layer of solid elements. This approach solves the drawbacks of using fully threedimensional models. It is similar to the single-layer approach since an integration rule is defined to represent the layers of the laminate [19].

\subsection{Material Models}

Over the years, LS-DYNA has implemented a wide variety of composite material models. The study of Schweizerhof et al. [20] provides an explanatory overview of some of them. There are models that degrade the mechanical properties to reproduce the damage of the material and those that only study the elastic response of the composite (no failure modes). A summary of the most used composite material models is provided in Table 2.1, 2.2 and 2.3 [1].

Progressive and continuous methods are used to degrade the elastic properties of the composite material. Progressive damage is obtained by discounting failed plies along the laminate, and continuum damage is represented by the inclusion of 
Table 2.1.

Composite models in LS-DYNA - Progressive failure degradation law.

\begin{tabular}{|c|l|c|c|c|}
\hline \multirow{2}{*}{ MAT } & \multicolumn{1}{|c|}{ Title } & Solid & $\begin{array}{c}\text { Thin } \\
\text { shell }\end{array}$ & $\begin{array}{c}\text { Thick } \\
\text { shell }\end{array}$ \\
\hline 22 & MAT_COMPOSITE_DAMAGE & o & o & o \\
\hline $54 / 55$ & MAT_ENHANCED_COMPOSITE_DAMAGE & & o & \\
\hline 59 & MAT_COMPOSITE_FAILURE_option_MODEL & o & o & \\
\hline
\end{tabular}

Table 2.2.

Composite models in LS-DYNA - Damage mechanics degradation law.

\begin{tabular}{|c|l|c|c|c|}
\hline MAT & \multicolumn{1}{|c|}{ Title } & Solid & $\begin{array}{c}\text { Thin } \\
\text { shell }\end{array}$ & $\begin{array}{c}\text { Thick } \\
\text { shell }\end{array}$ \\
\hline 58 & MAT_LAMINATED_COMPOSITE_FABRIC & & o & o \\
\hline 158 & MAT_RATE_SENSITIVE_COMPOSITE_FABRIC & & o & o \\
\hline 161 & MAT_COMPOSITE_MSC & o & & \\
\hline 162 & MAT_COMPOSITE_MSC_DMG & o & o & \\
\hline
\end{tabular}

Table 2.3.

Composite models in LS-DYNA - No failure models.

\begin{tabular}{|c|l|c|c|c|}
\hline MAT & Title & Solid & $\begin{array}{c}\text { Thin } \\
\text { shell }\end{array}$ & $\begin{array}{c}\text { Thick } \\
\text { shell }\end{array}$ \\
\hline 116 & MAT_COMPOSITE_LAYUP & & o & \\
\hline 117 & MAT_COMPOSITE_MATRIX & & o & \\
\hline 118 & MAT_COMPOSITE_DIRECT & & o & \\
\hline
\end{tabular}

micro-cracks and cavities into the material. Continuum damage models represent a smoother degradation than progressive damage models [20]. 


\subsubsection{MAT_054/055: Enhanced Composite Damage Model}

MAT_054/055, also known as Enhanced Composite Damage Material Model, is a progressive damage model that is widely used in the analysis of shell-like structures made of orthotropic materials, e.g., the unidirectional plies of composite laminates [21]. This is the material model used in this work. MAT_054/055 has been widely applied in the study of large-scale structural problems because it is computationally efficient. It requires few parameters for the definition of orthotropic materials. Crash simulations using MAT_054/055 have successfully reproduced experimental results of many studies [22], [17].

An explanatory review of MAT_054/055 is found in [23] and [24]. Those references in combination with the LS-DYNA's Manual of Material Models [21] are recommended to gain more insight about MAT_054/055.

The material parameters can be classified as constitutive, ply failure (damage onset), damage factors, ply deletion, element deletion, shear weighting and failure criteria as shown in Fig. 2.1 [23] [24].

\section{Constitutive parameters [23]}

In MAT_054/055, the stress-strain behavior in the elastic zone along the longitudinal direction (a-direction/fiber), transverse direction (b-direction/matrix) and shear direction (ab-direction) is defined as follows,

$$
\begin{aligned}
& \varepsilon_{a a}=\frac{1}{E_{a}}\left(\sigma_{a a}-\nu_{a b} \sigma_{b b}\right) \\
& \varepsilon_{b b}=\frac{1}{E_{b}}\left(\sigma_{b b}-\nu_{b a} \sigma_{a a}\right) \\
& 2 \varepsilon_{a b}=\frac{1}{G_{a b}} \sigma_{a b}-\alpha \sigma_{a b}{ }^{3}
\end{aligned}
$$




\begin{tabular}{|c|c|c|c|c|c|c|c|c|}
\hline \multicolumn{9}{|c|}{ *MAT ENHANCED_COMPOSITE DAMAGE } \\
\hline S & $\overline{\mathrm{MID}}$ & RO & EA & EB & $-\mathrm{EC}-$ & PRBA & -PRCA- & -PRBA- \\
\hline & 1 & $1.50 \mathrm{E}-9$ & $1.24 \mathrm{E}+5$ & 8411.60 & & 0.02049 & & \\
\hline S & GAB & GBC & GCA & $-\mathrm{KF}-$ & AOPT & & & \\
\hline & 4205.80 & 4205.80 & 4205.80 & & & & & \\
\hline S & XP & YP & ZP & A1 & $\mathrm{A} 2$ & A3 & MANGLE & \\
\hline S & V1 & $\mathrm{V} 2$ & V3 & D1 & $\mathrm{D} 2$ & D3 & $\begin{array}{c}\text { DFAILM } \\
0.0240\end{array}$ & $\begin{array}{c}\text { DFAILS } \\
0.0300\end{array}$ \\
\hline S & TFAIL & ALPH & SOFT & FBRT & YCFAC & DFAILT & DFAILC & EFS \\
\hline & $1.15 \mathrm{E}-9$ & 0.10 & 0.57 & 0.50 & 1.20 & 0.0174 & -0.0116 & 0.0 \\
\hline S & $\begin{array}{c}\mathbf{X C} \\
1447.9\end{array}$ & $\underset{2164.96}{\mathbf{X T}}$ & $\begin{array}{c}\text { YC } \\
198.569\end{array}$ & $\begin{array}{c}\mathbf{Y T} \\
48.883\end{array}$ & $\begin{array}{c}\text { SC } \\
154.443\end{array}$ & $\begin{array}{c}\text { CRIT } \\
54\end{array}$ & $\begin{array}{c}\text { BETA } \\
0.500\end{array}$ & \\
\hline
\end{tabular}

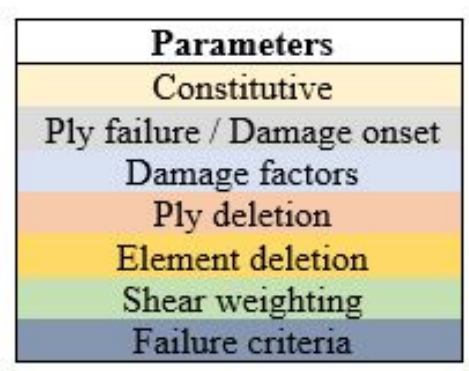

Figure 2.1. MAT_054/055 input card example

The coefficient $\alpha$ of Eq. 2.3 is a weighting factor for the non-linear shear stress. The weighting factor is not determined experimentally but by calibration of the FE model.

\section{Ply failure (damage onset) $[1,21,23]$}

The material strengths define the ply failure and the onset of the degradation of elastic properties. The difference between Materials 54 and 55 is the failure criteria used to limit the ply stresses that start the degradation process. Material 54 uses Chang-Chang criteria and Material 55 implements Tsi-Wu criteria. The degradation scheme reduces certain elastic properties to zero when a failure criterion has been reached.

The degradation process for Material 54 (Chang-Chang failure criteria) is given as follows, 
Tensile fiber mode:

$$
\sigma_{a a}>0 \rightarrow e_{f}^{2}=\left(\frac{\sigma_{a a}}{X_{t}}\right)^{2}+\beta\left(\frac{\sigma_{a b}}{S_{c}}\right)-1 \begin{cases}\geq 0 & \text { failed } \\ <0 & \text { elastic }\end{cases}
$$$$
\text { If failed: } E_{a}=E_{b}=G_{a b}=\nu_{b a}=\nu_{a b}=0
$$

Compressive fiber mode:

$$
\begin{aligned}
\sigma_{a a}<0 \rightarrow e_{c}^{2}= & \left(\frac{\sigma_{a a}}{X_{c}}\right)^{2}-1 \begin{cases}\geq 0 & \text { failed } \\
<0 & \text { elastic }\end{cases} \\
& \text { If failed: } E_{a}=\nu_{b a}=\nu_{a b}=0
\end{aligned}
$$

Tensile matrix mode:

$$
\sigma_{b b}>0 \rightarrow e_{m}^{2}=\left(\frac{\sigma_{b b}}{Y_{t}}\right)^{2}+\left(\frac{\sigma_{a b}}{S_{c}}\right)^{2}-1 \begin{cases}\geq 0 & \text { failed } \\ <0 & \text { elastic }\end{cases}
$$

$$
\text { If failed: } E_{b}=\nu_{a b}=0 \rightarrow G_{a b}=0
$$

Compressive matrix mode:

$$
\begin{array}{r}
\sigma_{b b}<0 \rightarrow e_{d}^{2}=\left(\frac{\sigma_{b b}}{2 S_{c}}\right)^{2}+\left[\left(\frac{Y_{c}}{2 S_{c}}\right)^{2}-1\right] \frac{\sigma_{b b}}{Y_{c}}+\left(\frac{\sigma_{a b}}{S_{c}}\right)^{2}-1 \begin{cases}\geq 0 & \text { failed, } \\
<0 & \text { elastic }\end{cases} \\
\text { If failed: } E_{b}=\nu_{b a}=\nu_{a b}=0 \rightarrow G_{a b}=0
\end{array}
$$

Material 55 implements Tsi-Wu criteria. The Tsi-Wu criteria for the tensile and compressive fiber modes are the same as the Chang-Chang criteria, but the tensile and compressive matrix modes are different. They are given by the expression:

$$
e_{m d}^{2}=\frac{\sigma_{b b}^{2}}{Y_{c} Y_{t}}+\left(\frac{\sigma_{a b}}{S_{c}}\right)^{2}+\frac{\left(Y_{c}-Y_{t}\right) \sigma_{b b}}{Y_{c} Y_{t}}-1 \begin{cases}\geq 0 & \text { failed } \\ <0 & \text { elastic }\end{cases}
$$$$
\text { If failed: } E_{b}=\nu_{b a}=\nu_{a b}=0 \rightarrow G_{a b}=0
$$ 


\section{Damage factors $[23]$}

Damage factors are used to degrade the strengths of the material.

If compressive matrix failure occurs, FBRT and YCFAC reduce the tensile and compressive strengths of the fiber, $\mathrm{XT}$ and $\mathrm{XC}$, as follows,

$$
\begin{aligned}
& \mathrm{XT}_{\text {reduced }}=\mathrm{XT} \cdot \mathrm{FBRT} \\
& \mathrm{XC}_{\text {reduced }}=\mathrm{YC} \cdot \mathrm{YCFAC}
\end{aligned}
$$

Where YC is the transversal compressive strength of the material.

In crash events, the material strengths are reduced by the SOFT factor as follows,

$$
\{\mathrm{XT}, \mathrm{XC}, \mathrm{YT}, \mathrm{YC}\}_{\text {reduced }}=\{\mathrm{XT}, \mathrm{XC}, \mathrm{YT}, \mathrm{YC}\} \cdot \mathrm{SOFT}
$$

The FBRT, YCFAC and SOFT parameters cannot be determined experimentally but by calibration of the FE model.

\section{Ply deletion [23]}

The deletion of the plies is controlled by five strain values. Once one ply has reached one of those values, it is deleted from the laminate. Of the five deletion strains, four can be determined experimentally. They are the failure strains in the positive fiber direction (tension) DFAILT, in the negative fiber direction (compression) DFAILC, in the matrix direction (transverse failure strain) DFAILM, and in shear DFAILS. The last value corresponds to the Effective Failure Strain (EFS). It is given by:

$$
\mathrm{EFS}=\sqrt{\frac{4}{3}\left(\varepsilon_{a a}^{2}+\varepsilon_{a a} \varepsilon_{b b}+\varepsilon_{b b}^{2}+\varepsilon_{a b}^{2}\right)}
$$

\section{Element deletion [23]}

In MAT_054/055, a shell element is deleted when all its plies, represented by integration points, have reached one of the failure strains. 
The time parameter, TFAIL, leads to the deletion of shell elements that are highly distorted to avoid numerical instabilities. It is given by,

TFAIL $\leq 0: \quad$ No element deletion by time step

$0<$ TFAIL $\leq 0.1: \quad$ Element deletion when its time step is smaller than TFAIL

TFAIL $>0.1 \quad$ Element is deleted when $\frac{\text { current time step }}{\text { original time step }}<$ TFAIL

\subsection{Basic LS-DYNA Keywords for Composites Analysis}

Once the material model has been selected, there are other basic keywords that must be included in the LS-DYNA input deck to perform composite analysis. The purpose of this section is to describe some of them.

LS-DYNA provides many options to define the layup (stacking sequence) of a laminate. Table 2.4 shows the three most common ways.

Table 2.4 .

Keywords options to define a laminate in LS-DYNA.

\begin{tabular}{|c|c|}
\hline Option & Keywords \\
\hline 1 & SECTION_SHELL and INTEGRATION_SHELL \\
\hline 2 & PART_COMPOSITE \\
\hline 3 & ELEMENT_SHELL_COMPOSITE \\
\hline
\end{tabular}

In this study, the composite laminates are defined using SECTION_SHELL and INTEGRATION_SHELL.

\subsubsection{INTEGRATION_SHELL}

INTEGRATION_SHELL card is used to define an integration rule through the thickness of the shell element. The layers of the laminate are represented by each integration point. To define laminates whose layers have the same material and 
thickness, ESOP (equal spacing of integration points option) is set to 1. ESOP is set to 0 when working with hybrid laminates (plies with different materials) or plies with different thicknesses. In those cases an integration point coordinate $(\mathrm{S})$ and a weighting factor must be defined [25].

\subsubsection{SECTION_SHELL}

In SECTION_SHELL card, the integration rule that represents the laminate layup is included in the field QR/IRID (Quadrature rule or Integration rule ID). The total thickness of the laminate is written in the shell thickness box (T1). Finally, the fiber orientation of each ply is defined by setting the ICOMP (Flag for orthotropic/anisotropic layered composite material model) to 1. Once ICOMP has been set to 1, a table to define the fiber orientations is activated as shown in Fig. 2.2.

\begin{tabular}{|c|c|c|c|c|c|c|c|c|c|c|c|}
\hline \multicolumn{12}{|c|}{ *SECTION_SHELL_(TITLE) (15) } \\
\hline \multicolumn{12}{|c|}{ IITLE } \\
\hline \multicolumn{12}{|c|}{ 1_ply } \\
\hline \multirow[t]{2}{*}{1} & $\underline{\text { SECID }}$ & ELFORM & $\underline{\text { SHRF }}$ & $\underline{\text { NIP }}$ & $\underline{\text { PROPT }}$ & & $\underline{Q R / I R I D}$ & $\underline{\text { ICOMP }}$ & & $\underline{\text { SETYP }}$ & \\
\hline & 1 & 16 & 1.0000000 & 1 & 1 & & \begin{tabular}{|l|}
-1 \\
\end{tabular} & - 1 & & 1 & $\checkmark$ \\
\hline 2 & $\underline{\mathrm{I}}$ & $\underline{\mathrm{I}}$ & $\underline{\mathrm{T}}$ & $\underline{\mathrm{T} 4}$ & $\underline{N L O C}$ & & MAREA & IDOF & & EDGSET & \\
\hline & 0.5000000 & 0.5000000 & 0.5000000 & 0.5000000 & 0.0 & & 0.0 & 0.0 & & 0 & \\
\hline \multicolumn{12}{|c|}{ Repeated Data by Button and List } \\
\hline & $\underline{B i}$ & $\underline{B i}$ & Bi & $\underline{B i}$ & $\underline{B i}$ & & $\underline{B i}$ & $\underline{B i}$ & & $\underline{B i}$ & \\
\hline & 15.0 & -15.0 & 15.0 & -15.0 & 15.0 & & -15.0 & 15.0 & & $\mid-15.0$ & \\
\hline & 1 1 15:0-15 & $5.015 .0-1.1$ & $0-15.0$ & & & & & Data & & & \\
\hline
\end{tabular}

Figure 2.2. Example of SECTION_SHELL keyword.

\subsubsection{PART_COMPOSITE}

PART_COMPOSITE card is a simplified option to define a composite material for shell and thick shell elements. It couples the functionalities of the SECTION_SHELL and INTEGRATION_SHELL cards. In PART_COMPOSITE, the thickness, fiber orientation and material of each layer is defined in a table (Fig. 2.3). The laminate thickness is the sum of the thickness of each integration point [25]. 


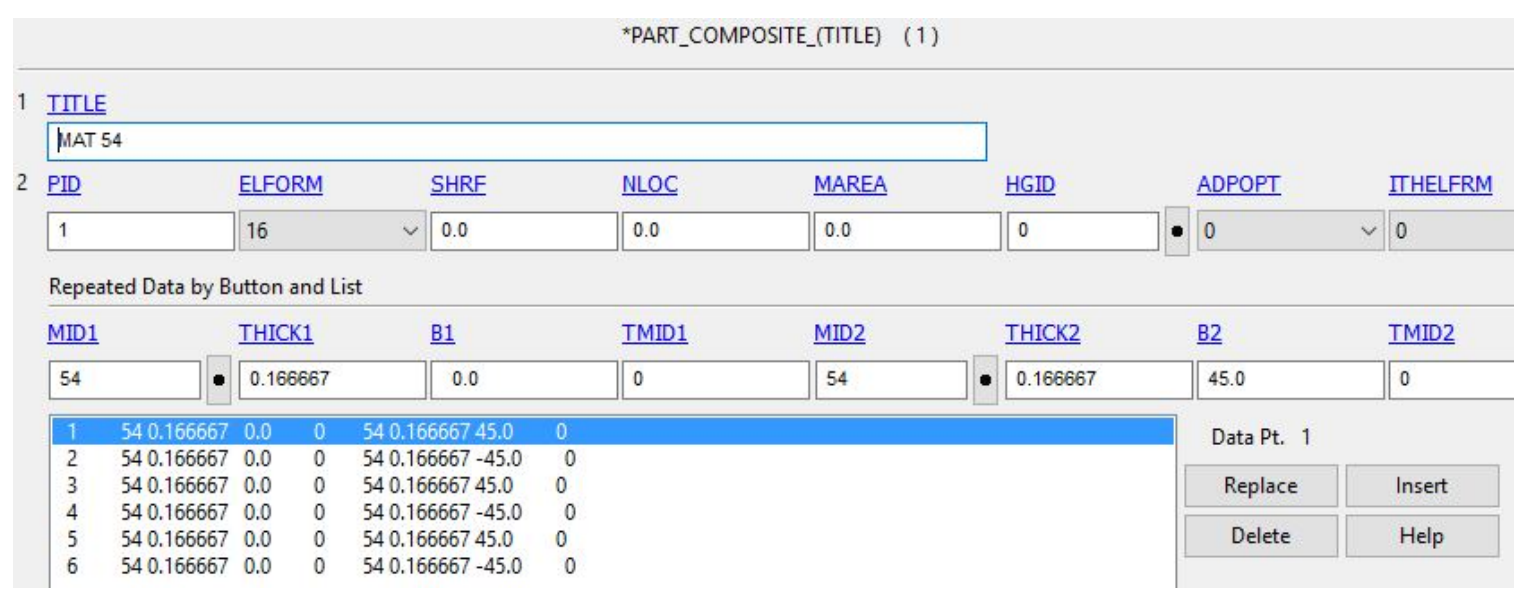

Figure 2.3. Example of PART_COMPOSITE keyword.

\subsubsection{ELEMENT_SHELL_COMPOSITE}

Another option to define a composite laminate is to use the ELEMENT_ SHELL_ COMPOSITE keyword. As in PART_COMPOSITE, the thickness, fiber orientation and material of each layer is defined in a table. ELEMENT_SHELL_COMPOSITE card can be used when the composite material need to be defined in a shell-element basis form.

\subsubsection{CONTROL_ACCURACY}

The material coordinate system of orthotropic elements is highly sensitive to inplane shearing and hourglass deformations. The CONTROL_ACCURACY card contains the Invariant Node Numbering (INN) option. When this option is activated (2 for shell elements, 3 for solid elements, and 4 for both shell and solid elements), the material coordinate system is automatically updated as the simulation progresses [1]. The INN effect is illustrated in Fig. 2.4. 


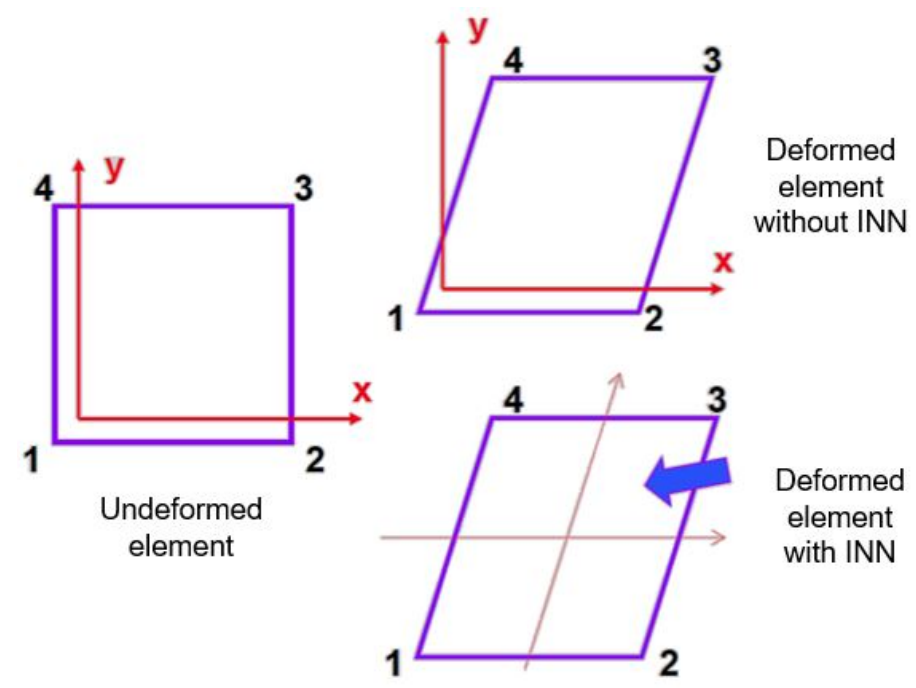

Figure 2.4. Effect of CONTROL_ACCURACY over the material coordinates of deformed shell elements.

\subsubsection{CONTROL_SHELL}

CONTROL_SHELL provide several options to modify the response of shell elements. In composites analysis, Laminated Shell Theory (LST) is activated (set to 1) to correct the assumption that shear stress is uniform and constant through the laminate thickness. This correction is important when there are notable differences in the elastic properties among plies [25].

\subsubsection{DAMPING_PART_STIFFNESS}

According to Andersson [26] as well as LS-DYNA developers [25], stiffness damping is an effective form of controlling nonrealistic failure in high-velocity impact simulations with composites. A Rayleigh stiffness damping between 0.01 and 0.25 is recommended [25]. 


\section{SURROGATE-BASED GLOBAL OPTIMIZATION}

\subsection{Metamodel Generation}

There are several mathematical techniques for the construction of surrogates models. In the case of impact analysis, polynomials, response surface, radial basis functions, multivariate adaptive regression splines (MARS) and (Gaussian Process) Kriging have been used succesfully $[27,28]$.

Kriging metamodel is used in this work since it provides high accuracy, facility to perform optimization and design space exploration [15,16,27]. Additionally, Kriging provides estimations of the accuracy of its predictions. This feature enables the possibility of using predictions' uncertainties to direct the optimization towards the global optimums while the metamodel is continuously enhanced along the searching process.

\subsubsection{Kriging}

Kriging metamodel, also known as Gaussian Process interpolation, is a surrogate model to approximate deterministic data [29]. Kriging surrogates have extensively applied in global optimization, design space exploration, variable screening, visualization of input-output interactions and tradeoff analysis between competing functions [16]. To gain more insight about the methodology, an extensive mathematical analysis of Kriging surrogates is developed in [15]. Due to the popularity of Kriging among researchers, it has been extended to other applications such as stochastic problems, and the combination of high and low fidelity information [30]. Various Matlab implementations of Kriging surrogates have been developed [29,30]. Among them, the ooDACE Matlab toolbox is used in this work [30]. 
Kriging prediction $Y(x)$ can be studied as a two-step process: $f(x)$ and $Y(x)$. In this process, $f(x)$ is a function that represents the main trend of the data and $Z(x)$ is a Gaussian Process that refines the prediction by using residuals information. Kriging prediction is defined as follow,

$$
Y(x)=f(x)+Z(x)
$$

where $f(x)$ is a regression (or trend) function and $Z(x)$ is a Gaussian process with mean 0 , variance $\sigma^{2}$ and correlation matrix $\Psi$ [30].

Depending on the form of the regression function, Kriging acquires different denominations. It is Simple Kriging if the regression function is assumed to be a known constant, e.g., $f(x)=0$. A more popular version is Ordinary Kriging. It assumes a constant regression function but with unknown value $f(x)=\alpha_{0}$. If necessesary, more complex functions can be defined like Universal Kriging that defines the regression function as a multivariate polynomial [30] as follow,

$$
f(x)=\sum_{i=1}^{p} \alpha_{i} b_{i}(x)
$$

where $\left(\alpha_{1}, \ldots, \alpha_{p}\right)$ are the regression coefficients and $\left(b_{1}(x), \ldots, b_{p}(x)\right)$ are the basis functions of the polynomial.

Considering a set of $n$ samples, $X=\left\{x^{1}, \ldots, x^{n}\right\}$ in $d$ dimensions whose function values are $\mathrm{y}=\left\{y^{1}, \ldots, y^{n}\right\}$. The regression function and stochastic process are mainly represented by the $n \times p$ model matrix $F$ and the $n \times n$ correlation matrix $\Psi$, respectively.

$$
\begin{gathered}
F=\left[\begin{array}{ccc}
b_{1}\left(x^{1}\right) & \ldots & b_{p}\left(x^{1}\right) \\
\vdots & \vdots & \vdots \\
b_{1}\left(x^{n}\right) & \ldots & b_{p}\left(x^{n}\right)
\end{array}\right] \\
\Psi=\left[\begin{array}{ccc}
\psi\left(x^{1}, x^{1}\right) & \ldots & \psi\left(x^{1}, x^{n}\right) \\
\vdots & \ddots & \vdots \\
\psi\left(x^{n}, x^{1}\right) & \ldots & \psi\left(x^{n}, x^{n}\right)
\end{array}\right]
\end{gathered}
$$


where $\psi(\cdot, \cdot)$ is the correlation function with hyperparameters $\theta$.

Depending on the regression function $f(x)$, the Kriging predictor takes different forms. Its general form is,

$$
\hat{y}(x)=M \alpha+r(x)^{T} \Psi^{-1}(\mathrm{y}-F \alpha)
$$

where $M=\left(b_{1}(x) b_{2}(x) \ldots b_{p}(x)\right)$ is a $1 \times p$ vector that contains the values of the basis functions evaluated at predicting point $x, \alpha$ is the vector of regression coefficients, $\alpha=\left(F^{T} \Psi^{-1} F\right)^{-1} F^{T} \Psi^{-1}$ y. $r(x)=\left(\psi\left(x, x^{1}\right) \ldots \psi\left(x, x^{n}\right)\right)^{T}$ is a $n \times 1$ vector that contains the correlation values between the predicting point $x$ and the samples $X$.

A popular version of the Kriging predictor is the Best Linear Unbiased Predictor of Eq. 3.4. This form is utilized in the work of Jones et. al [16] for surrogated-based optimization.

$$
\hat{y}(x)=\hat{\mu}+r(x)^{T} \Psi^{-1}(\mathrm{y}-\mathbf{1} \hat{\mu})
$$

where $\hat{\mu}$ is the response of the regression function for $x$, and $\mathbf{1}$ is a $n \times 1$ vector of ones. The variance of the Kriging prediction is defined as,

$$
s^{2}(x)=\sigma^{2}\left(1-r(x)^{T} \Psi^{-1} r(x)+\frac{\left(1-\mathbf{1}^{T} \Psi^{-1} r(x)\right)^{2}}{\mathbf{1}^{T} \Psi^{-1} \mathbf{1}}\right)
$$

The variance of the stochastic process, $\sigma^{2}, \hat{\mu}$, and the hyperparameters $\theta$ of the correlation function are determined by Maximum Likelihood Estimation (MLE) [16]. The variance is an estimation of the accuracy of the prediction.

It is important to say that Eq. 3.3 and 3.4 define Kriging as an interpolation model. Therefore, if $\hat{y}(x)$ is evaluated at a sample point $x^{i}$, the prediction must be equal to $y^{i}$, and the prediction variance $s^{2}\left(x^{i}\right)$ must be equal to zero since no error is expected (high correlation of the evaluated point) as shown in Fig 3.1. 


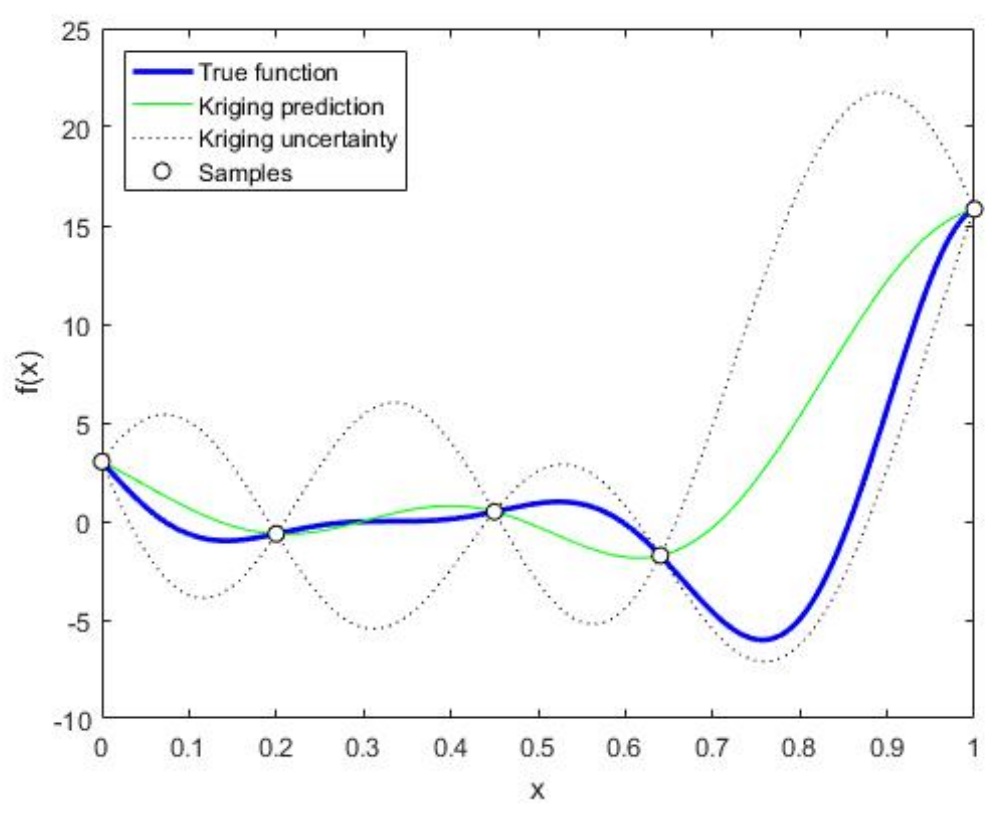

Figure 3.1. Kriging predictions and uncertainties.

In order to demonstrate that Kriging is an interpolation technique, a sample point $x^{i}$ is used. If $x=x^{i}$ is evaluated in $r(x), r(x)$ will be equivalent to the $i$ th column of $\Psi$. Then, the result of $r(x)^{T} \Psi^{-1}$ is,

$$
r(x)^{T} \Psi^{-1}=\left(\Psi^{-1} r(x)\right)^{T}=\left(\Psi^{-1} \Psi_{i}\right)^{T}=e_{i}^{T}
$$

where $e_{i}$ is a $i$ th unit vector.

Now, it is possible to demonstrate that Kriging is an interpolation model if Eq. 3.6 is replaced in Eq. 3.4,

$$
\hat{y}\left(x_{i}\right)=\hat{\mu}+e_{i}(\mathrm{y}-\mathbf{1} \hat{\mu})=y^{i}
$$

The effect of the correlation in the Kriging prediction is illustrated in Fig. 3.2. If a point $x^{*}$, close to the sample point $x^{(2)}$, is evaluated. It is expected that the prediction of $x^{*}$ is going to be close to $y^{(2)}$ because there is high correlation between $x^{*}$ and $x^{(2)}$. Additionally, the prediction of $x^{*}$ has high accuracy due to its proximity to a sample point.

The lowest variance, occurs when $x$ is a sample point. If $x=x_{i}$, it follows that, 


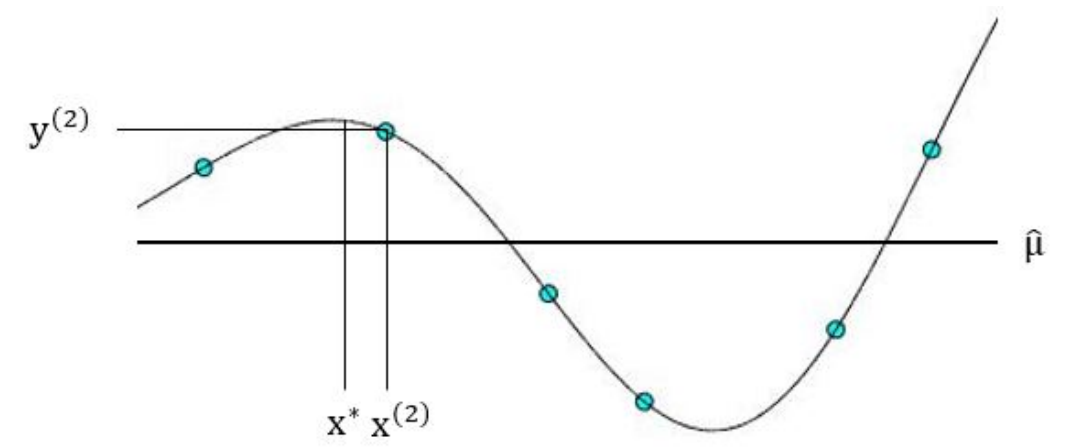

Figure 3.2. Illustration of how correlation affects Kriging prediction. The prediction of $\mathrm{x}^{*}$, that is close to the sample point $\mathrm{x}^{(2)}$, should be adjusted from the regression line to take into account the positive residual at $\mathrm{x}^{(2)}$.

$$
r\left(x^{i}\right)^{T} \Psi^{-1} r\left(x_{i}\right)=r\left(x_{i}\right)^{T} e_{i}=\psi\left(x_{i}, x_{i}\right)=1
$$

and,

$$
\mathbf{1}^{T} \Psi^{-1} r\left(x_{i}\right)=\mathbf{1}^{T} e_{i}=1
$$

If Eq. 3.8 and 3.9 are replaced into 3.5, $s^{2}\left(x_{i}\right)=0$. This shows that accurate predictions are obtained when the predicting point $x$ is near to the samples $X$.

\section{Correlation functions $[16,30]$}

Although there are several options to define the correlation function $\psi\left(x, x^{\prime}\right)$, it must be chosen adequately in order to obtain accurate Kriging surrogates. A commonly correlation function is defined by

$$
\begin{aligned}
d\left(x, x^{\prime}\right) & =\sum_{i=1}^{d} \theta_{i}\left|x_{i}-x_{i}^{\prime}\right|^{p_{i}} \\
\psi\left(x, x^{\prime}\right) & =\exp \left[-d\left(x, x^{\prime}\right)\right]
\end{aligned}
$$

Eq. 3.10 represents a weighted distance between the points $x$ and $x^{\prime} . \theta_{i}$ and $p_{i}$ are the hyperparameters of the correlation function that are estimated by MLE. 
Eq. 3.11 reflects the dependency of the correlation with respect to the distance $d\left(x, x^{\prime}\right)$. The function shows that there is high correlation between points when the distance is small. Therefore, the more influence those points have in the prediction. Conversely, when the distance increases, the correlation function drops to zero with a rate that depends on the hyperparameters.

The parameters $p_{i}$ reflects the smoothness of the model. Commonly $p_{i}$ is set to two (Gaussian correlation). A value of two is used under the assumption that the data represents a smooth function. If the Kriging metamodel represents less smooth functions, a value of one is used (Exponential correlation). Fig. 3.3 illustrates the effect of $p_{i}$ (the higher the value, the smoother the correlation function).

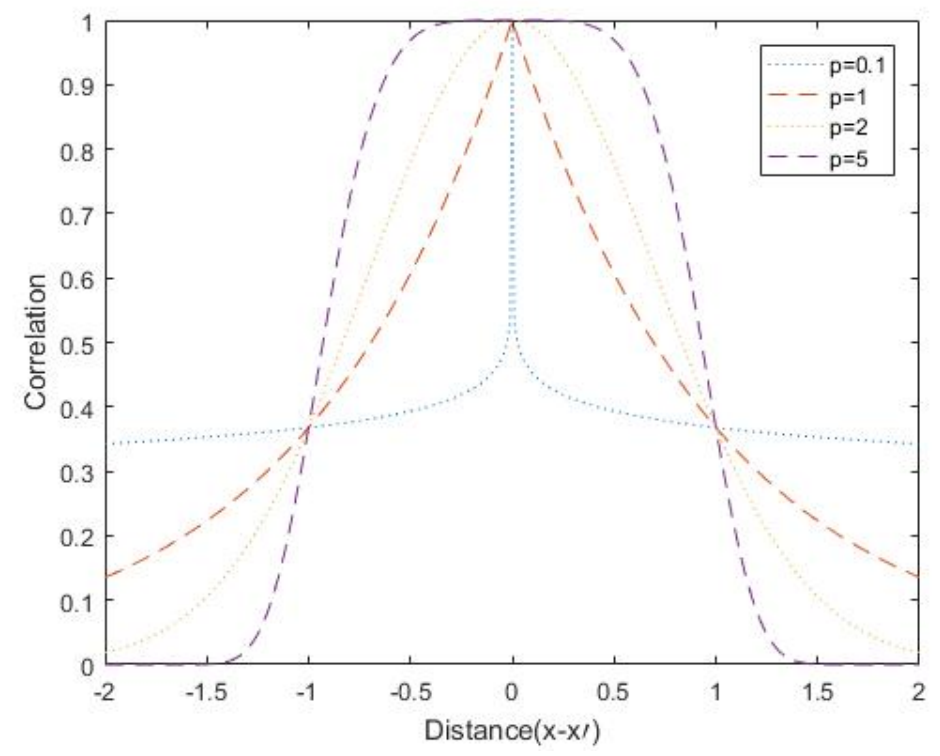

Figure 3.3. Effect of $p_{i}$ in the correlation function.

The remaining set of parameters $\theta_{i}$ have values higher than zero, and measure the activity of the $i$ th component of $x$ and $x^{\prime}$. Large values of $\theta_{i}$ means that the $i$ th components are active since even small distance values lead to large changes in the response. Statistically, it means that there is a low correlation between points even with small values of $\left|x_{i}-x_{i}^{\prime}\right|$. The hyperparameters $\theta_{i}$ determine how fast the correlation function drops to zero as illustrated in Fig. 3.4. 


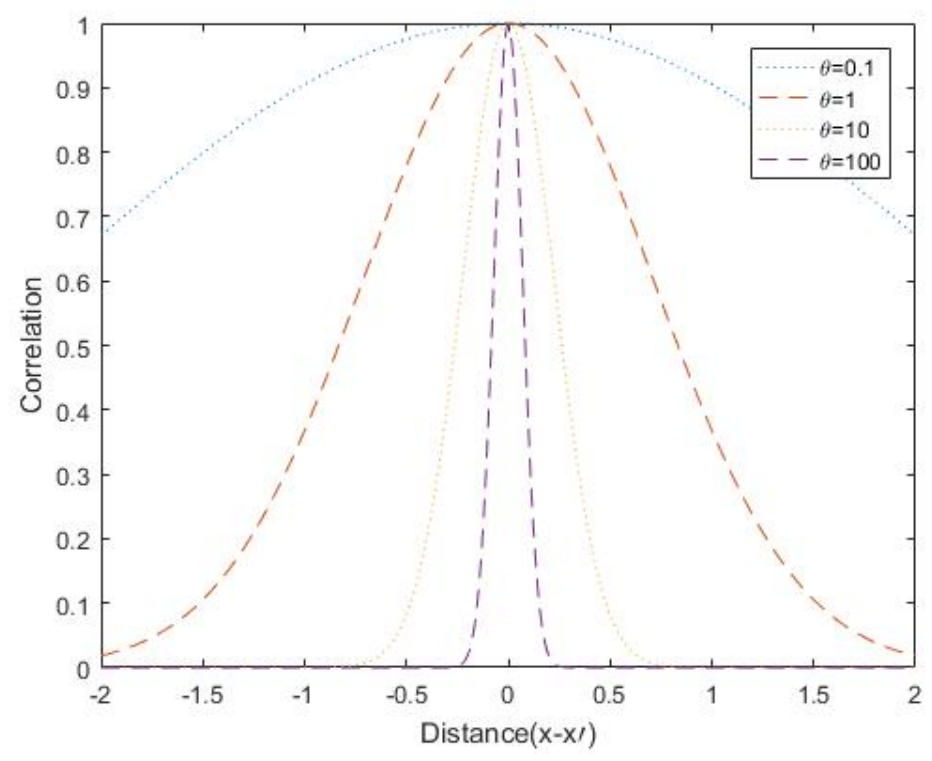

Figure 3.4. Effect of $\theta_{i}$ in the correlation function.

As stated before the values $\theta_{i}$ and $p_{i}$ are determined in the MLE. Commonly, $p_{i}$ is predefined and fixed and only the parameters $\theta_{i}$ are calculated.

\section{Maximum Likelihood Estimation [16]}

Once the regression and correlation functions have been chosen, the next step is to determine the model parameters $\mu, \sigma, \theta_{1}, \ldots, \theta_{d}$, and $p_{1}, \ldots, p_{d}$. The Concentrated Likelihood Function can be maximized in the MLE.

$$
\mathcal{L}=\frac{1}{(2 \pi)^{n / 2}\left(\sigma^{2}\right)^{n / 2}|\Psi|^{1 / 2}} \exp \left[-\frac{(\mathrm{y}-\mathbf{1} \mu)^{T} \Psi^{-1}(\mathrm{y}-\mathbf{1} \mu)}{2 \sigma^{2}}\right]
$$

Eq.3.12 is the likelihood function of the sample. Being $d$ the dimension of the design space, the likelihood function has $2 d+d$ variables $\left(\mu, \sigma, \theta_{1}, \ldots, \theta_{d}, p_{1}, \ldots, p_{d}\right)$ that need to be determined. $\Psi$ is the correlation matrix of the sample points, and y is the column vector of the sample responses.

If the hyperparameters $\theta_{1}, \ldots, \theta_{d}$, and $p_{1}, \ldots, p_{d}$ are predefined, the likelihood function can be maximized with respect to $\mu$ and $\sigma$ leading to the expressions, 


$$
\hat{\mu}=\frac{\mathbf{1}^{T} \Psi^{-1} \mathrm{y}}{\mathbf{1}^{T} \Psi^{-1} \mathbf{1}}
$$

and

$$
\hat{\sigma}^{2}=\frac{(\mathrm{y}-\mathbf{1} \hat{\mu})^{T} \Psi^{-1}(\mathrm{y}-\mathbf{1} \hat{\mu})}{n}
$$

The Concentrated Likelihood Function (CLF) is obtained when Eq. 3.13 and 3.14 are replaced in Eq. 3.12. CLF is maximized to find the hyperparameters of the correlation function $\Psi$. Then, the $\theta_{d}$, and $p_{d}$ estimates are used to update the values of $\hat{\mu}$ and $\hat{\sigma}^{2}$ using Eq. 3.13 and 3.14 again.

\subsubsection{Latin Hypercube Sampling}

Design and Analysis of Computer Experiments (DACE) arises in the scenario of selecting the sample points to be simulated in order to increase the accuracy of the surrogate model. In the case of expensive simulations, it is important to define a sampling strategy with space-filling properties. Methodologies like random sampling, stratified sampling and Latin hypercube sampling (LHS) can be used [29, 31].

Among the mentioned options, it has been proved that LHS provides samples with better space-filling properties. This design strategy ensures that all the portions of the design space are represented in a stratified manner by a given number of sample points $P[32]$. LHS is used in this work to generate the initial Kriging metamodels.

The components of the LHS matrix are defined as,

$$
S_{p k}=\frac{\eta_{p k}-0.5}{P}
$$

where $P$ is the number of sample points, $k$ is the design space dimension, and $\eta_{1 k}, \ldots, \eta_{p k}$ are uniform random permutations of the integers 1 through $P$. 
Although Eq. 3.15 defines sample points with excellent space-filling properties, they can have undesirable correlations. There are several approaches to reduce the correlation of the sample points, e.g., maximization of the minimum inter-site distances $[15,33]$ and correlation minimization methods [34]. The correlation minimization is implemented in this work to generate the LHS. Fig. 3.5 shows a high correlation LHS and an optimized LHS with correlation minimization method.
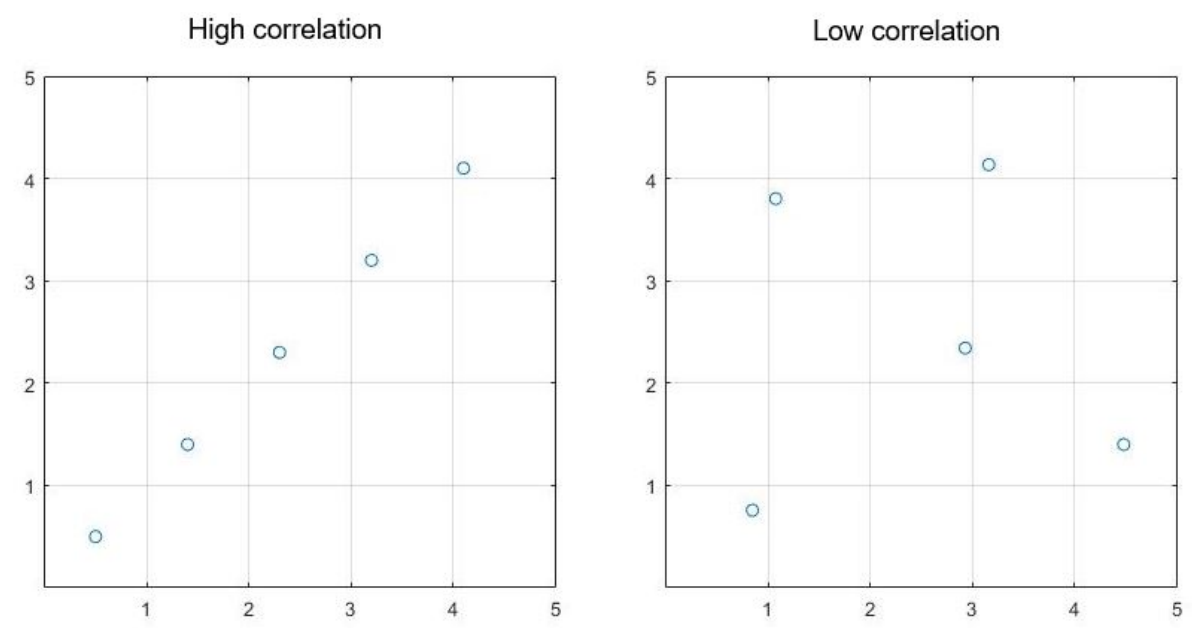

Figure 3.5. LHS with $\mathrm{P}=5$. High correlation sampling and low correlation sampling due to correlation minimization method.

\subsubsection{Model Validation}

A common approach to estimate the accuracy of a surrogate is to select a few additional points to compare the metamodel prediction and the simulation response. This validation method is not viable when dealing with expensive simulations. In such cases, cross-validation is preferred since it does not require additional simulations. The metamodel accuracy is evaluated using the data of the already simulated samples [33].

Cross-validation involves grouping the sample responses randomly. Then, one design, named $y\left(x^{i}\right)$, is removed from the $P$ samples. Finally, a $P-1$ surrogate with the remaining designs is created. Next, the error between the actual response and the prediction of the $P-1$ surrogate at sample $x^{i}$ is calculated, $e_{p}=\left|y\left(x^{i}\right)-y_{P-1}\left(x^{i}\right)\right|$. 
The process is repeated for all the samples to determine the PRESS vector [15,35]. Fig. 3.6 illustrates cross-validation at the second sample.

$$
\operatorname{PRESS}=\mathbf{e}^{T} \mathbf{e}
$$

The PRESS vector is the predicted residual error sum of squares (PRESS). It contains all the cross-validated errors $e_{p}$. The root-mean square error of the PRESS

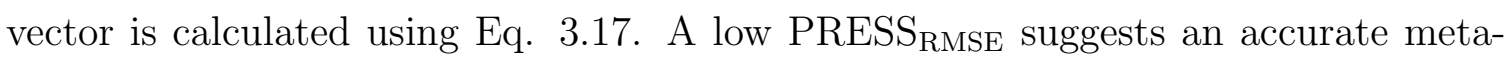
model. This is the metric used to validate the metamodels of this study and select the initial metamodel for the optimization.

$$
\operatorname{PRESS}_{\mathrm{RMSE}}=\sqrt{\frac{1}{P} \mathbf{e}^{T} \mathbf{e}}
$$

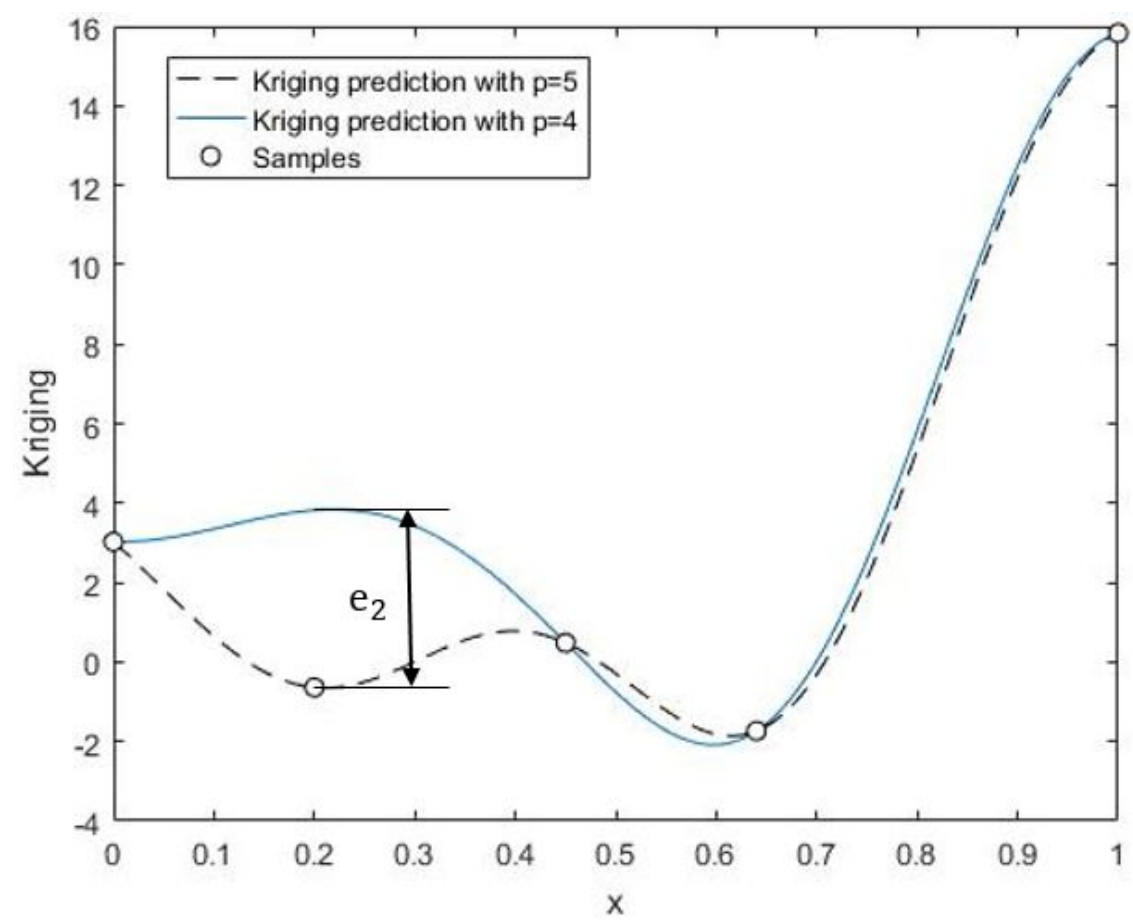

Figure 3.6. Cross-validation at the second sampled point. 


\subsection{Metamodel-Based Global Optimization}

Once the Kriging metamodels have been constructed and validated, the optimization algorithm is implemented. In this work, the Efficient Global Optimization (EGO) algorithm proposed by Jones et al. [16] is applied. EGO provides an excellent scheme for surrogate-based global optimization since it balances metamodel exploitation and design space exploration. EGO continuously improves the metamodel while searching for the global optimum by selecting new samples that maximize the Expected Improvement function of the surrogate at each iteration $[15,16]$.

\subsubsection{Expected Improvement}

The Expected Improvement (EI) function calculates the amount of improvement that is expected if a design $x$ is sampled. EI functions is defined as,

$$
E[I(x)]=E\left[\max \left(f_{\min }-F, 0\right)\right]
$$

where $f_{\min }=\min \left\{y^{1}, \ldots, y^{n}\right\}$ is the best current value of the sample, and $F$ is a normally distributed random variable with mean and variance provided by the Kriging surrogate $\left(\hat{y}\right.$ and $\left.s^{2}\right)$. The closed form of the expected improvement is found by replacing $F=\operatorname{Normal}\left(\hat{y}, s^{2}\right)$ in Eq. 3.18 and integrating the right side [16].

$$
E[I(x)]=\left(f_{\min }-\hat{y}\right) \Phi\left(\frac{f_{\min }-\hat{y}}{s}\right)+s \phi\left(\frac{f_{\min }-\hat{y}}{s}\right)
$$

where $\hat{y}=\hat{y}(x)$ is the predicted value at $x, s$ is the root square of the variance of the Kriging prediction. $\Phi(\cdot)$ is the cumulative density function (CDF), and $\phi(\cdot)$ is the probability density distribution (PDF).

The expected improvement of a sample in any place of the design space can be evaluated using Eq. 3.19. Fig. 3.7 illustrates the EI function of the Kriging surrogate with nine sample points. According to the figure, the metamodel is improved if points 
$x=0.142$ and $x=0.704$ are sampled. By sampling in this manner, the optimizer balances between metamodel exploitation and design space exploration.

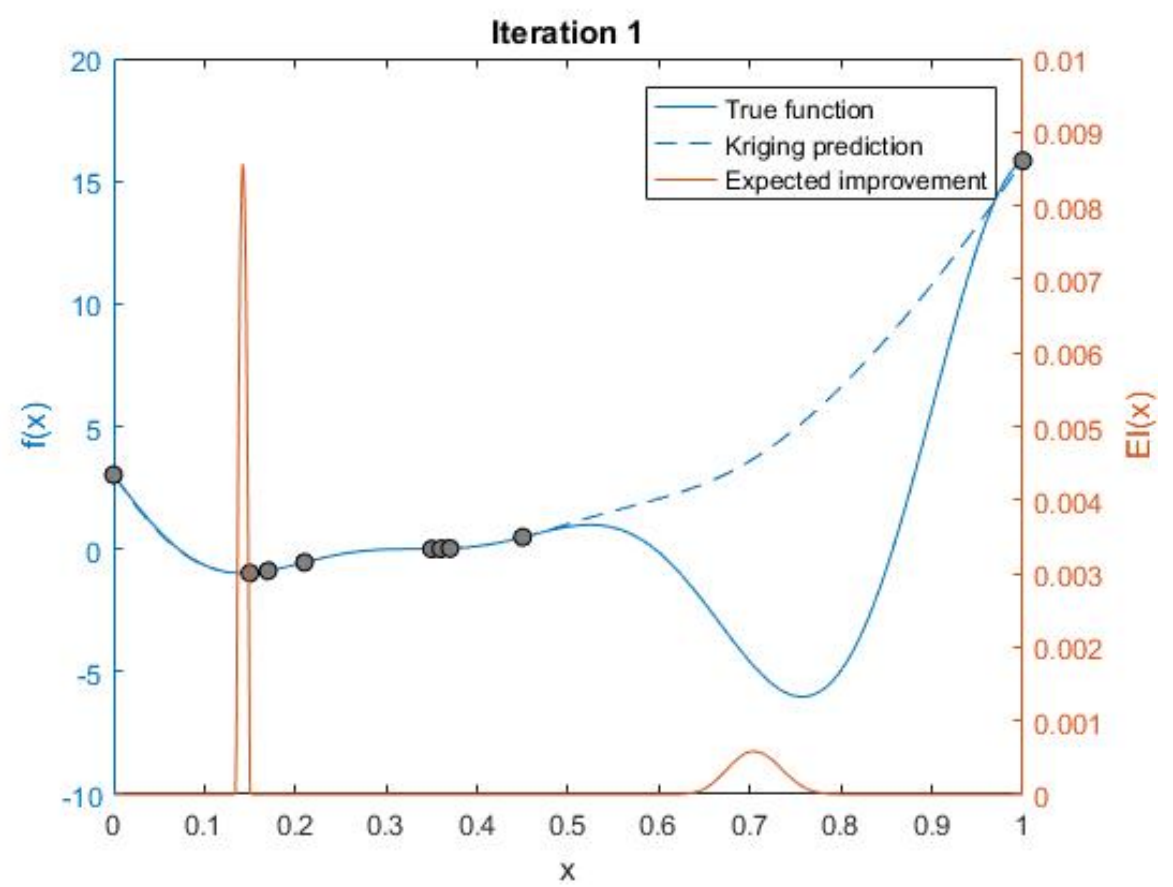

Figure 3.7. Expected improvement function with nine samples. The left scale is for the objective function and the right scale for the expected improvement.

Fig. 3.8 shows the progress of the optimization process using maximization of the EI function. The global optimizer is used to find the design that maximizes the EI function. In this manner, the search leads to designs with promising performance (minimum responses) as well as designs that improve the accuracy of areas with few data (high uncertainty areas). As a result, the metamodel is continuously enhanced while the optimizer searches for the global optimum [15].

When dealing with constrained problems, the aim of balancing exploration and exploitation remains, but it is also required to include the feasibility of the new design points. The EI function is modified to take into account the feasibility of new samples by defining a constrained expected improvement (CEI) function. One option for CEI is to assign an expected improvement value of zero to the unfeasible designs if they can be determined directly. In this way, the optimizer only searches in feasible areas. 

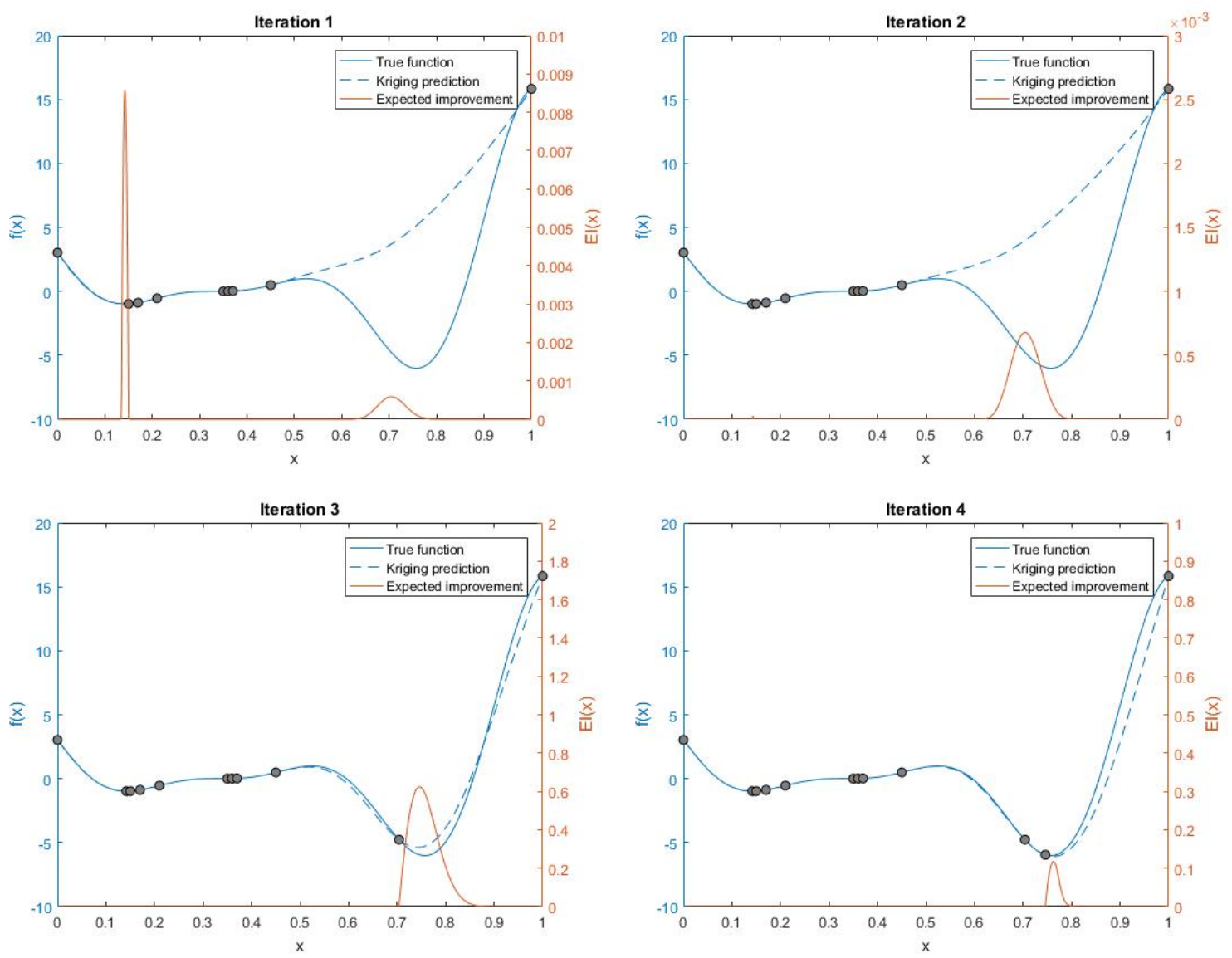

Figure 3.8. Progress of the optimization process when the designs that maximize the EI are sampled.

In some cases it is not possible to determine which designs are feasible and which are not. To deal with such scenarios, surrogates for the model responses (objective functions) and the constraints are created. Those surrogates provide error information that is used to define the CEI as follow [15].

$$
E[I(x) \cap F(x)]=E[I(x)] P[F(x)]
$$

Eq. 3.20 is the CEI function. $E[I(x)]$ is the expected improvement of a design $x$ and $P[F(x)]$ is the probability that the constraint is satisfied. 


\subsubsection{Multi-Objective Expected Improvement [15]}

Commonly, engineering design involves solving problems with multiple, conflicting objectives and demanding constraints. In such cases, it is necessary to define a multiobjective expected improvement (MEI) function for the optimization algorithm.

Considering a problem with two objective functions, $f_{1}(x)$ and $f_{2}(x)$, that need to be minimized, e.g., weight and impact force. It is possible to sample a design $x$ to obtain the responses $y_{1}$ and $y_{2}$. By evaluating a sample plan $\mathbf{X}$, the response vectors $\mathbf{y}_{\mathbf{1}}$ and $\mathbf{y}_{\mathbf{2}}$ are formed. Then, from those values an initial Pareto front (set of $m$ non-dominated responses) is identified.

$$
\mathbf{y}_{\mathbf{1}, \mathbf{2}}^{*}=\left\{\left(y_{1}^{*(1)}, y_{2}^{*(1)}\right),\left(y_{1}^{*(2)}, y_{2}^{*(2)}\right), \ldots,\left(y_{1}^{*(m)}, y_{2}^{*(m)}\right)\right\}
$$

where $y_{j}^{*(i)}=f_{j}\left(x^{*(i)}\right)$ and $x^{*(i)}$ is a Pareto design.

The expected improvement for the multi-objective problem is defined as:

$$
E\left[I\left(x^{*(p)}\right)\right]=P\left[I\left(x^{*(p)}\right)\right] \min \left(d_{1}, \ldots, d_{m}\right)
$$

where $P\left[I\left(x^{*(p)}\right)\right]$ is the probability of improving both functions $f_{1}(x)$ and $f_{2}(x)$ with a design $x^{*(p)}$. This probability is calculated by integrating over the area to the left and below the Pareto front of the two-dimensional probability density function. $\min \left(d_{1}, \ldots, d_{m}\right)$ determines the minimum Euclidean distance between one Pareto design and the probability integral centroid, $\left(\bar{F}_{1}, \bar{F}_{2}\right)$, which lies below and to the right of the Pareto front. Designs with high expected improvement will tend to either augment or expand the Pareto front.

The probability of improvement is defined as:

$$
P\left[I\left(x^{*(p)}\right)\right]=\Phi\left(u_{1}^{i}\right)+\sum_{i=1}^{m-1}\left[\Phi\left(u_{1}^{i+1}\right)-\Phi\left(u_{1}^{i}\right)\right] \Phi\left(u_{2}^{i+1}\right)+\left[1-\Phi\left(u_{1}^{m}\right)\right] \Phi\left(u_{2}^{m}\right)
$$


where $u_{j}^{i}=u_{j}^{i}\left(x^{* p}\right)=\left(y_{j}^{*(i)}-\hat{y}_{j}\left(x^{*(p)}\right)\right) / \sigma_{j}\left(x^{*(p)}\right)$. The values of $\hat{y}_{j}\left(x^{*(p)}\right)$ and $\sigma_{j}\left(x^{*(p)}\right)$ correspond to the prediction and variance calculated using the Kriging surrogate.

The Euclidean distance between a member of the Pareto front, $\left(y_{1}^{*(i)}, y_{2}^{*(i)}\right)$, and the centroid of the probability integral is given by,

$$
\sqrt{\left(\bar{F}_{1}\left(x^{*(p)}\right)-y_{1}^{*(i)}\right)^{2}+\left(\bar{F}_{2}\left(x^{*(p)}\right)-y_{2}^{*(i)}\right)^{2}}
$$

The centroid locations are defined as,

$$
\bar{F}_{1}\left(x^{*(p)}\right)=\frac{1}{P\left[I\left(x^{*(p)}\right)\right]}\left[z_{1}^{1}+\sum_{i=1}^{m-1}\left(z_{1}^{i+1}-z_{1}^{i}\right) \Phi\left(u_{2}^{i+1}\right)+z_{1}^{m} \Phi\left(u_{2}^{m}\right)\right]
$$

where $z_{j}^{i}=z_{j}^{i}\left(x^{*(p)}\right)=\hat{y}_{j}\left(x^{*(p)}\right) \Phi\left(u_{j}^{i}\right)-\sigma_{j}\left(x^{*(p)}\right) \phi\left(u_{j}^{i}\right)$. The value of $\bar{F}_{2}\left(x^{*(p)}\right)$ is defined similarly.

Fig. 3.9 is an illustration of the components involved in the MEI function.

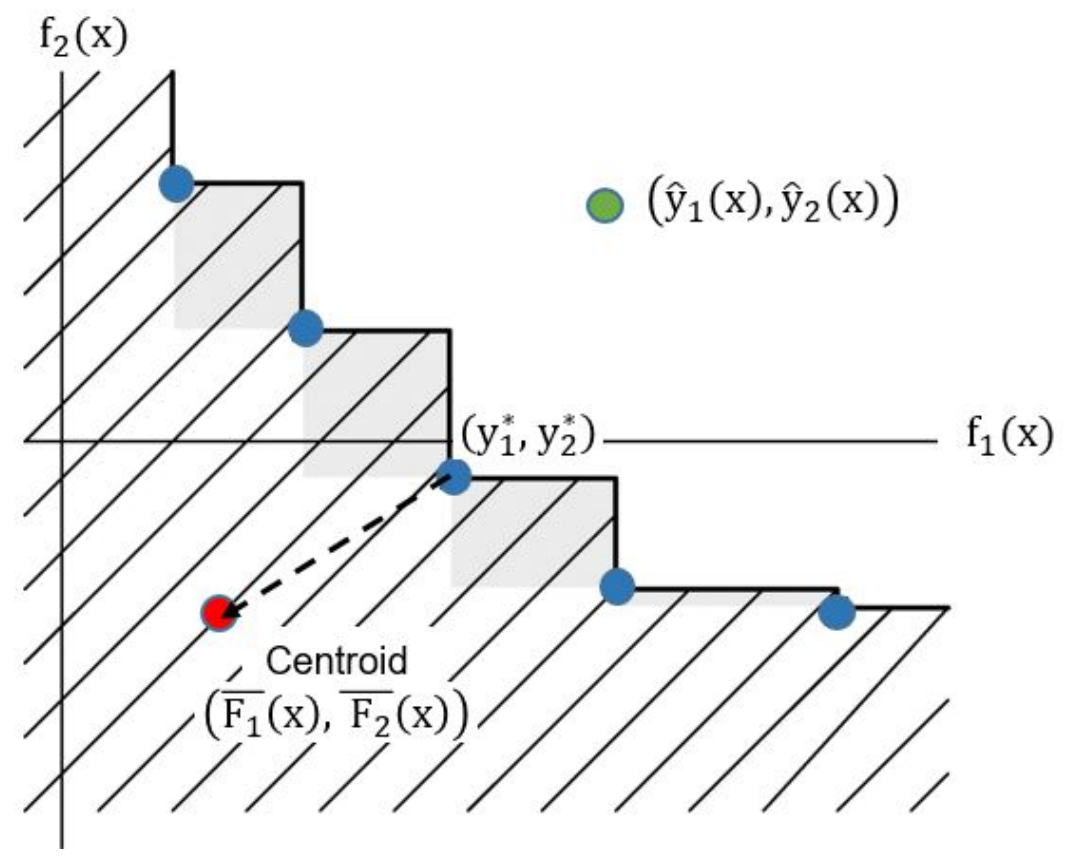

Figure 3.9. Multi-objective expected improvement. Centroid of the probability integral (red dot), Pareto front (blue dots) and predicted responses of sample $x$ (green dot). 


\subsubsection{The Efficient Global Optimization Algorithm}

The Global Efficient Global Optimization (EGO) algorithm proposed by Jones et. al [16] combines the principles of Kriging metamodel, Latin hypercube sampling, metamodel validation and expected improvement in order to perform the optimization process. It is described as follows,

Step 1. Intitial metamodel: The design space is sampled. The initial sample points are selected using Latin hypercube technique. In this work, the correlation minimization option provided by Matlab (lhsdesign) was used in order to improve the space-filling properties of the samples. A practical rule to select the number of samples is $P \simeq 10 \times k$. Where $P$ is the number of samples, and $k$ is the dimension of the design space [16].

After having simulated the sample designs, initial Kriging surrogates are created. The parameters of the metamodels are obtained by maximizing the likelihood of the sample (MLE).

Step 2. Metamodel validation: Cross-validation is used to estimate the accuracy of the Kriging surrogates. Root-mean square error (RMSE) of the predicted residual error sum of squares (PRESS) is used to estimate the accuracy of the metamodels. The metamodels with the lowest value is selected as the initial metamodel for the optimization.

Step 3. Metamodel-based global optimization: After the initial metamodel has been validated, the global optimization process is performed iteratively. The EI function is maximized at each iteration until a convergence criteria, e.g., if the improvement is less than a predefined value $(0.01 \%)$ the process is terminated. Otherwise, the Kriging surrogate is updated with the response of the sample point that maximizes the EI at each iteration. The process is repeated until convergence criteria are satisfied or the maximum number of iterations is consumed [16]. Fig. 3.10 illustrates EGO algorithm. 


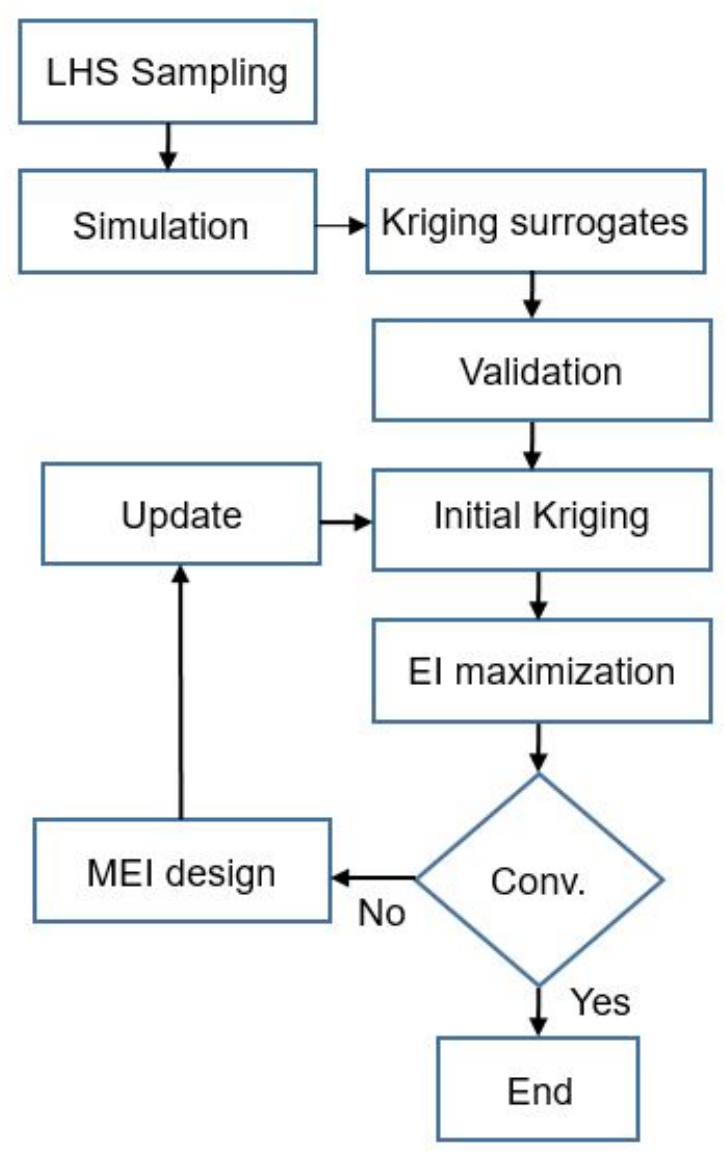

Figure 3.10. Efficient global optimization algorithm.

\subsection{Global Optimization of Composite Materials Parts under Dynamic Loading}

Although FE codes are capable of solving complex problems, it is difficult and often impractical to directly combine optimization algorithms and FE models since long-running simulation times are required. Also, reliable sensitivity coefficents are absent at the end of the simulation. To overcome those limitations, surrogate models are implemented in the optimization process. In this work, EGO algorithm performs the global optimization of composite parts under dynamic loading. MATLAB is used for generation of the sample designs, LS-DYNA interfacing, extraction of results from LS-DYNA output files and data analysis. The MATLAB - LS-DYNA implementation of EGO is illustrated in Fig. 3.11. 


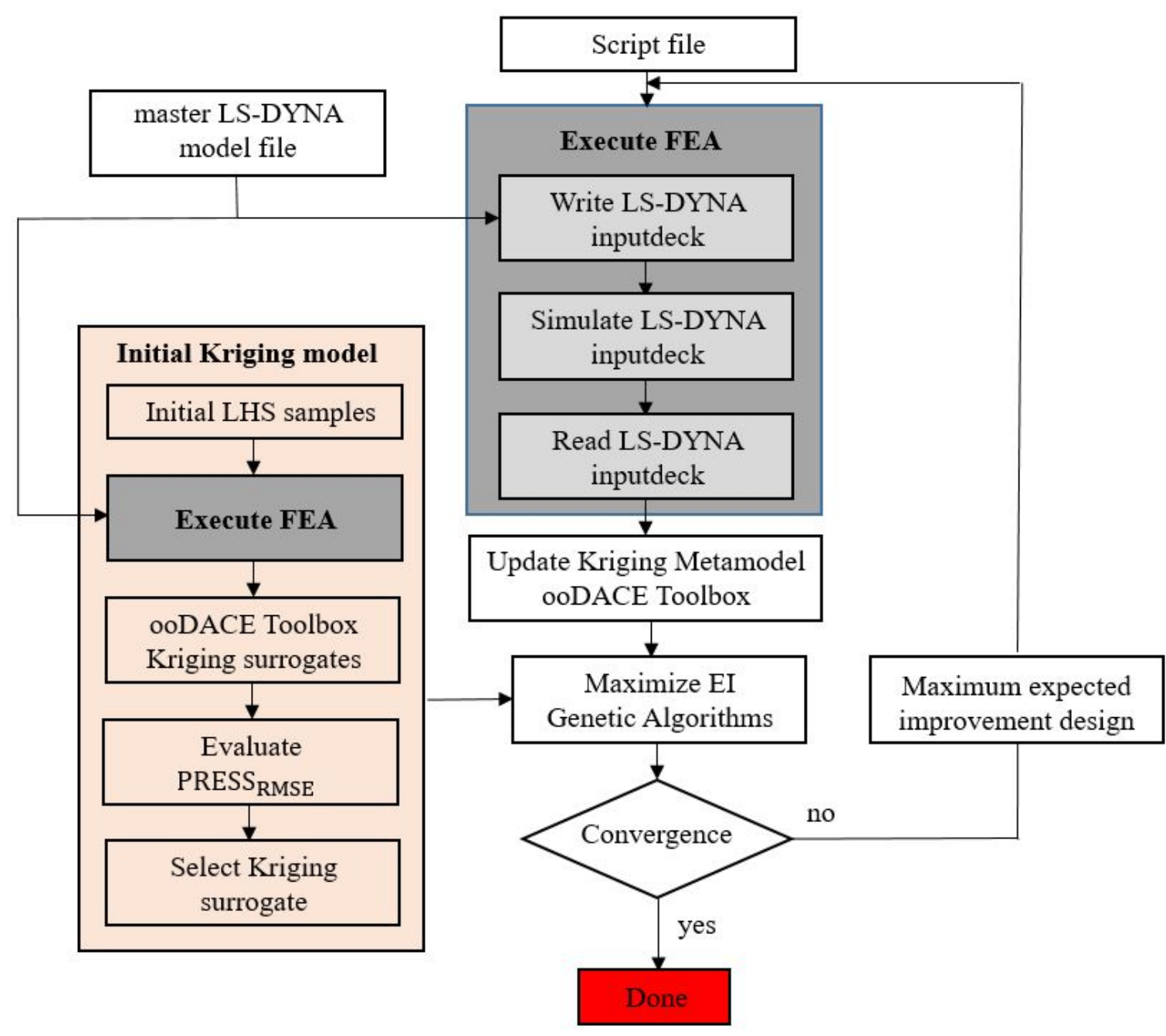

Figure 3.11. Efficient global optimization algorithm MATLAB - LS-DYNA.

In order to perform the optimization analysis of a particular problem, a master LS-DYNA input file is created. The master file defines the boundary conditions, simulation parameters and the design variables for the optimization problem. The master file is used to create input decks of the designs that are going to be sampled through the optimization process.

At the beginning, there are $P$ LHS samples whose designs are created and simulated. At the end of each simulation, the LS-DYNA output files are post-processed to extract the data for the initial Kriging metamodel. In the case of multi-objective 
optimization, more than one metamodel is created. The metamodel with the lower PRESS $_{\text {RMSE }}$ is selected as the initial metamodel for the optimization.

The EI is maximized to provide designs for metamodel updating or convergence evaluation. Genetic algorithms are used to perform the EI maximization since they are effective with non-linear and non-convex function. Additionally, they are well suited to work with mixed variable optimization problems [36].

If the maximum improvement is less than a predefined value, e.g. 0.01\%, convergence is achieved and the process is terminated. Otherwise, the master file is used to create a new input deck for simulation. The new file is based on the sample at which the EI is maximized.

The input file is simulated, the LS-DYNA output files are post-processed in order to update the Kriging metamodel. The process is repeated iteratively until either convergence or the maximum number of iterations are reached. 


\section{NUMERICAL EXAMPLES}

In this chapter four application examples are included to illustrate the use of EGO for the global optimization of composite material parts. The first two examples are single objective optimization problems, and the others are multi-objective optimization problems.

LS-DYNA material model MAT_054/055, known as MAT_ENHANCED_COMPOSITE_DAMAGE, is used in this work. The material parameters are shown in Table 4.1. The mechanical properties correspond to carbon fiber T700GF 12k/2510 unidirectional tape. Those values are taken from AGATE Design Allowables [37]. The addtional parameters are included in the study of Bonnie et. al [38].

The composite parts are modeled using shell formulation (ELFORM =16). This is a very efficient fully integrated shell formulation that does not undergo hour-glass effects (spurious strain energy modes) because of its four in-plane integration points scheme.

Regarding the impactors, they are modeled using MAT_RIGID_020. Their properties are included in Table 4.2 .

The contacts between the composite parts and the rigid bodies are modeled using CONTACT_AUTOMATIC_SURFACE_TO_SURFACE with a friction coefficient of 0.3 to allow sliding movement. CONTACT_SINGLE_SURFACE with a friction coefficient of 0.1 is also included to account for the contact among different composite parts.

The laminate layup is defined in the INTEGRATION_SHELL card. The material coordinate system is updated with CONTROL_ACCURACY (INN=2). Laminated shell theory is activated in CONTROL_SHELL (LST=1). Finally, a coefficient of 0.05 is defined in DAMPING_PART_STIFFNESS. 
Table 4.1.

MAT_054/055 parameters for application examples

\begin{tabular}{|c|c|c|c|}
\hline Variable & Property & Val & \\
\hline $\mathrm{RO}$ & Density & $1.500 \mathrm{E}-09$ & ton $/ \mathrm{mm}^{3}$ \\
\hline EA & Axial Young's modulus & $1.248 \mathrm{E}+05$ & $\mathrm{MPa}$ \\
\hline EB & Transverse Young's modulus & $8.412 \mathrm{E}+03$ & $\mathrm{MPa}$ \\
\hline GAB & Shear modulus & $4.206 \mathrm{E}+03$ & $\mathrm{MPa}$ \\
\hline PRBA & Minor Poisson's ratio & 0.02 & \\
\hline $\mathrm{XT}$ & Axial tensile strength & 2164.96 & $\mathrm{MPa}$ \\
\hline $\mathrm{XC}$ & Axial compressive strength & 1447.90 & $\mathrm{MPa}$ \\
\hline $\mathrm{YT}$ & Transverse tensile strength & 48.88 & $\mathrm{MPa}$ \\
\hline $\mathrm{YC}$ & Transverse compressive strength & 198.57 & $\mathrm{MPa}$ \\
\hline $\mathrm{SC}$ & Shear strength & 154.44 & $\mathrm{MPa}$ \\
\hline DFAILT & Axial tensile failure strain & 0.0174 & $\mathrm{~mm} / \mathrm{mm}$ \\
\hline DFAILC & Axial compressive failure strain & -0.0116 & $\mathrm{~mm} / \mathrm{mm}$ \\
\hline DFAILM & Transverse failure strain & 0.024 & $\mathrm{~mm} / \mathrm{mm}$ \\
\hline DFAILS & Shear failure strain & 0.03 & $\mathrm{~mm} / \mathrm{mm}$ \\
\hline EFS & Effective failure strain & 0 & $\mathrm{~mm} / \mathrm{mm}$ \\
\hline TFAIL & Time step failure value & 0 & $\mathrm{~s}$ \\
\hline FBRT & Fiber tension damage factor & 0.5 & \\
\hline YCFAC & Fiber compression damage factor & 1.2 & \\
\hline SOFT & Crush-front damage parameters & 0 & \\
\hline $\mathrm{ALPH}$ & Factor for the non-linear term in shear stress & 0.1 & \\
\hline BETA & Shear factor in fiber tension & 0.5 & \\
\hline
\end{tabular}

In the validation step, the surrogates of Table 4.3 are constructed and the one with

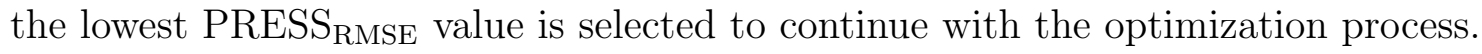


Table 4.2.

MAT_020 parameters for application examples

\begin{tabular}{|c|l|c|}
\hline Variable & \multicolumn{1}{|c|}{ Property } & \multicolumn{2}{|c|}{ Value } \\
\hline $\mathrm{E}$ & Young's modulus & $2.10 \mathrm{E}+05 \mathrm{MPa}$ \\
$\mathrm{PR}$ & Poisson's ratio & 0.30 \\
\hline
\end{tabular}

Table 4.3.

Kriging surrogate forms

\begin{tabular}{|c|c|c|}
\hline Surrogate & Regression function & Correlation function \\
\hline 1 & Zero-order polynomial & Gaussian \\
2 & First-order polynomial & Gaussian \\
3 & Second-order polynomial & Gaussian \\
4 & Zero-order polynomial & Exponential \\
5 & First-order polynomial & Exponential \\
6 & Second-order polynomial & Exponential \\
\hline
\end{tabular}

\subsection{Minimum Penetration under Dynamic Load on an Armor Plate}

In the following examples, a composite armor plate is impacted by a rigid sphere. The optimization algorithm will find the fiber orientations of the laminate that minimize the armor's penetration. Two scenarios are studied, symmetric and nonsymmetric impact.

The dimensions of the plate are $200 \times 200 \times 2 \mathrm{~mm}$ with fixed edges. The armor is made of a symmetric laminate (layup $=\left[\theta_{1} / \theta_{2}\right]_{s}$ ) of four plies. Each ply has a thickness of $0.5 \mathrm{~mm}$. For the ease of manufacturing the orientations are restricted to increments of 5 deg. between -90 and 90 deg.

The rigid sphere has a diameter of $60 \mathrm{~mm}$ and a mass of $0.25 \mathrm{~kg}$. It has an initial velocity of $10 \mathrm{~m} / \mathrm{s}$, perpendicular to the plate. 
For the numerical analysis, the plate has a mesh of $50 \times 50$ identical finite elements. The convergence criteria to terminate the optimization is an expected improvement less than $1 \times 10^{-3}$, and the maximum number of iterations is 150 .

The problem formulation is:

$$
\begin{array}{ll}
\text { find } & \boldsymbol{\theta} \in \mathbb{R}^{2} \\
\text { minimize } & d(\boldsymbol{\theta}) \\
\text { subject to } & \theta_{i} \in\left\{-90^{\circ},-85^{\circ}, \ldots, 85^{\circ}, 90^{\circ}\right\}
\end{array}
$$

where $\boldsymbol{\theta}=\left(\theta_{1}, \theta_{2}\right)$ are the fiber orientations, and $d(\boldsymbol{\theta})$ is the armor's penetration.

\subsubsection{Symmetric Impact}

In this loading case, the sphere impacts at the armor's center as illustrated in Fig. 4.1. The 21 LHS samples of Table 4.4 are used to create the initial Kriging metamodel for the armor's penetration, $\hat{d}(\boldsymbol{\theta})$ (Fig. 4.2).

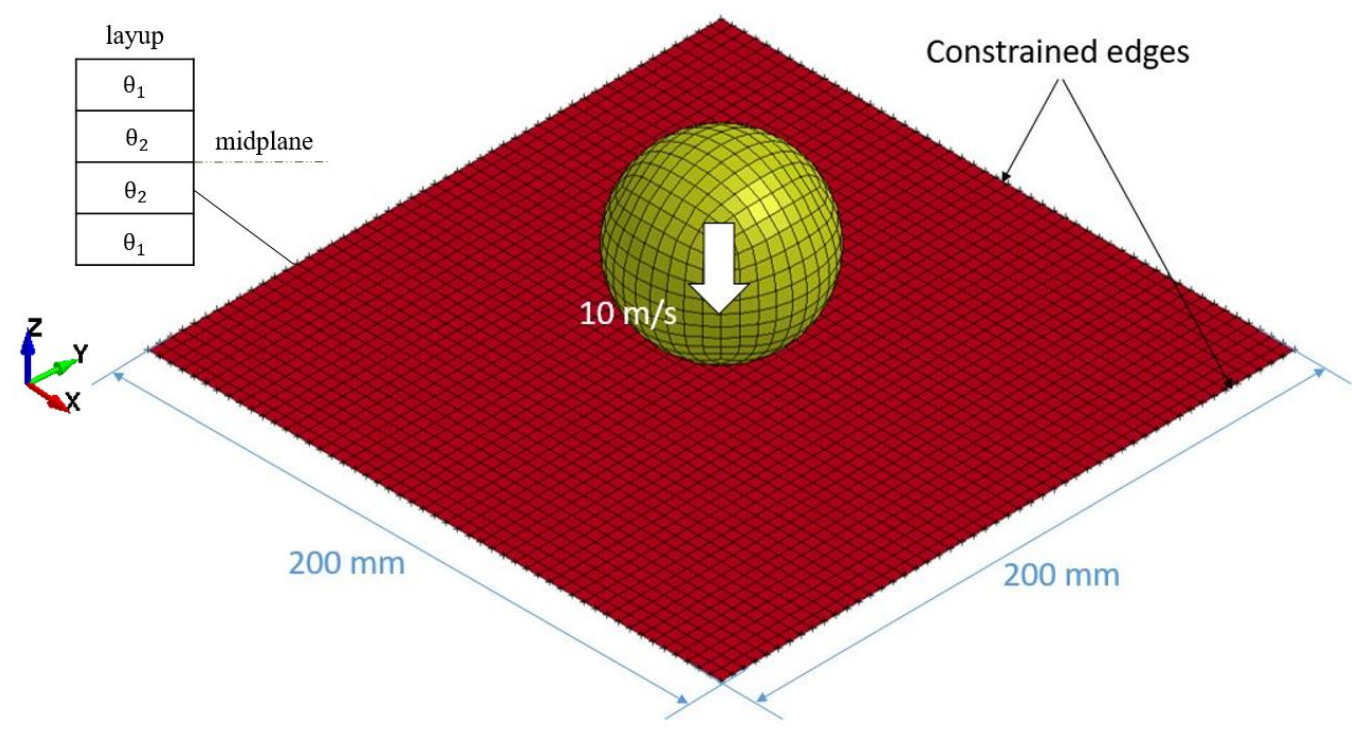

Figure 4.1. Armor plate problem - symmetric impact

According to Table 4.5, the most appropriate surrogate for the armor's penetration has a second-order regression function and exponential correlation. Its PRESS $\mathrm{RMSE}$ value is equal to $0.4769(\mathrm{~mm})$. 
Table 4.4 .

LHS sampling - Armor problem

\begin{tabular}{|cc|cc|cc|}
\hline$\theta_{1}$ (deg.) & $\theta_{2}$ (deg.) & $\theta_{1}$ (deg.) & $\theta_{2}$ (deg. $)$ & $\theta_{1}\left(^{\circ}\right)$ & $\theta_{2}\left({ }^{\circ}\right)$ \\
\hline 70 & -25 & 60 & 90 & -80 & 70 \\
-90 & 35 & -25 & 55 & 55 & -45 \\
0 & 20 & 35 & 80 & 10 & -80 \\
90 & 45 & -45 & -20 & 20 & 25 \\
-35 & -55 & 25 & -10 & 45 & -60 \\
-70 & -35 & -55 & 60 & -60 & -70 \\
-10 & 10 & -20 & -90 & 80 & 0 \\
\hline
\end{tabular}

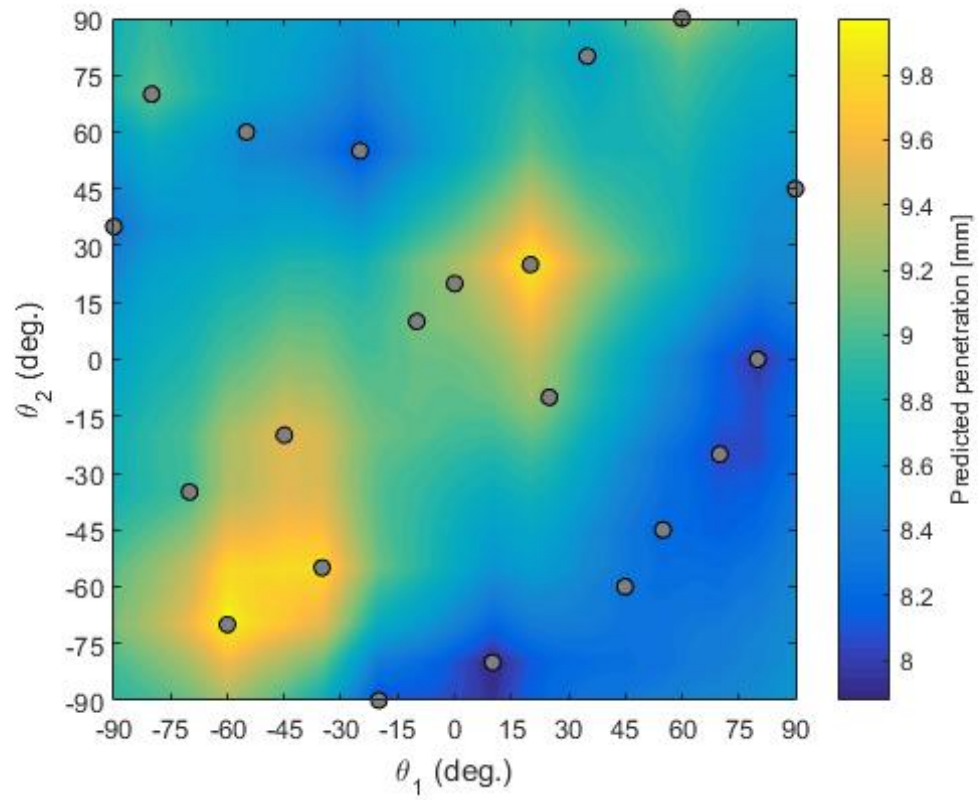

Figure 4.2. Armor problem - symmetric impact. Initial Kriging surrogate of the armor's penetration based on the 21 LHS sampled points shown as dots.

Fig. 4.3 and 4.4 show the first six EGO iterations. By the end of the optimization, the algorithm finds three optimal designs. Table 4.6 contains the number of iterations required to find each optimal design. The results suggest that a cross-ply laminate, $\left[0^{\circ} / 90^{\circ}\right]_{s}$, is the best option to minimize the armor's penetration. 
Table 4.5.

Armor plate problem - symmetric impact. PRESS RMSE $_{\text {of Kriging }}$ surrogates using different regression and correlation functions.

\begin{tabular}{|ccc|ccc|}
\hline \multicolumn{2}{|c|}{ Exponential correlation } & \multicolumn{3}{c|}{ Gaussian correlation } \\
\hline regpoly0 & regpoly1 & regpoly2 & regpoly0 & regpoly1 & regpoly2 \\
0.5735 & 0.6584 & 0.4769 & 0.4912 & 0.5866 & 0.4793 \\
\hline
\end{tabular}

Table 4.6.

Optimal designs armor problem, symmetric impact.

\begin{tabular}{|c|cc|c|}
\hline Iteration & $\theta_{1}(\mathrm{deg})$. & $\theta_{2}(\mathrm{deg})$. & Penetration $(\mathrm{mm})$ \\
\hline 8 & 0 & -90 & 7.8206 \\
20 & 90 & 0 & 7.8206 \\
49 & 0 & 90 & 7.8206 \\
\hline
\end{tabular}

Fig. 4.5 shows that the expected improvement continuously approach to zero after each iteration. The convergence criteria is satisfied at iteration 71 .

Fig. 4.6 shows the responses of the designs that are selected by EGO to update the Kriging surrogate at each iteration. Some samples are used to exploit the surrogate (low penetration values) while others are devoted to explore the design space (high penetration values). The optimal solutions (lowest penetration values) are found at iterations 8, 20 and 49.

Fig. 4.7 shows that EGO does not sample in the zones with the higher expected improvement values in the last iterations. This behavior happens because of the constraint on the degree-increments of the fiber orientation, $\left\{-90^{\circ},-85^{\circ}, \ldots, 90^{\circ}\right\}$. The zones that are not sampled correspond to unfeasible orientations. 

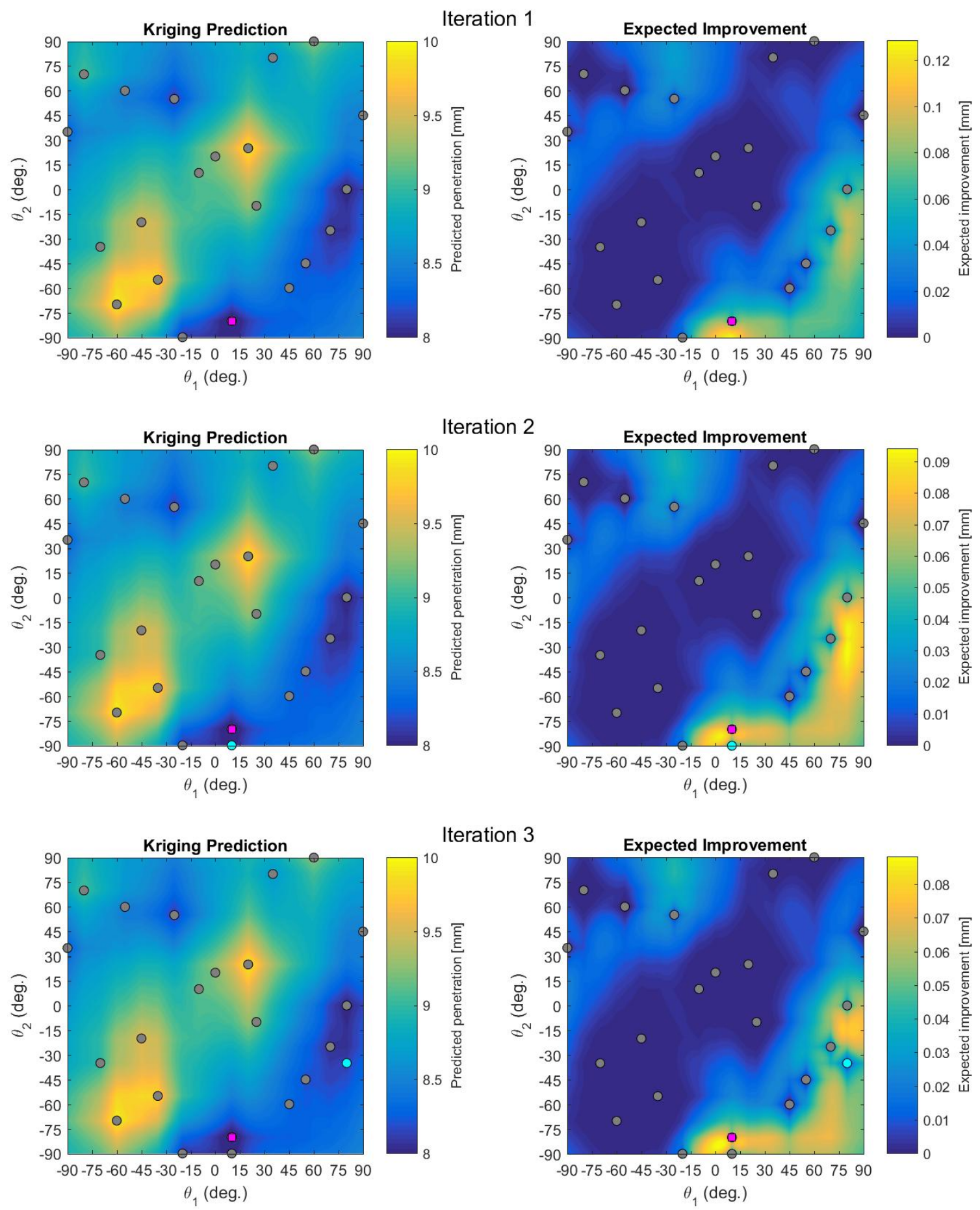

Figure 4.3. EGO optimization progress - Armor problem, symmetric impact. Current sample (grey), best current design (purple), last sampled design (cyan). Iterations 1, 2 and 3. 

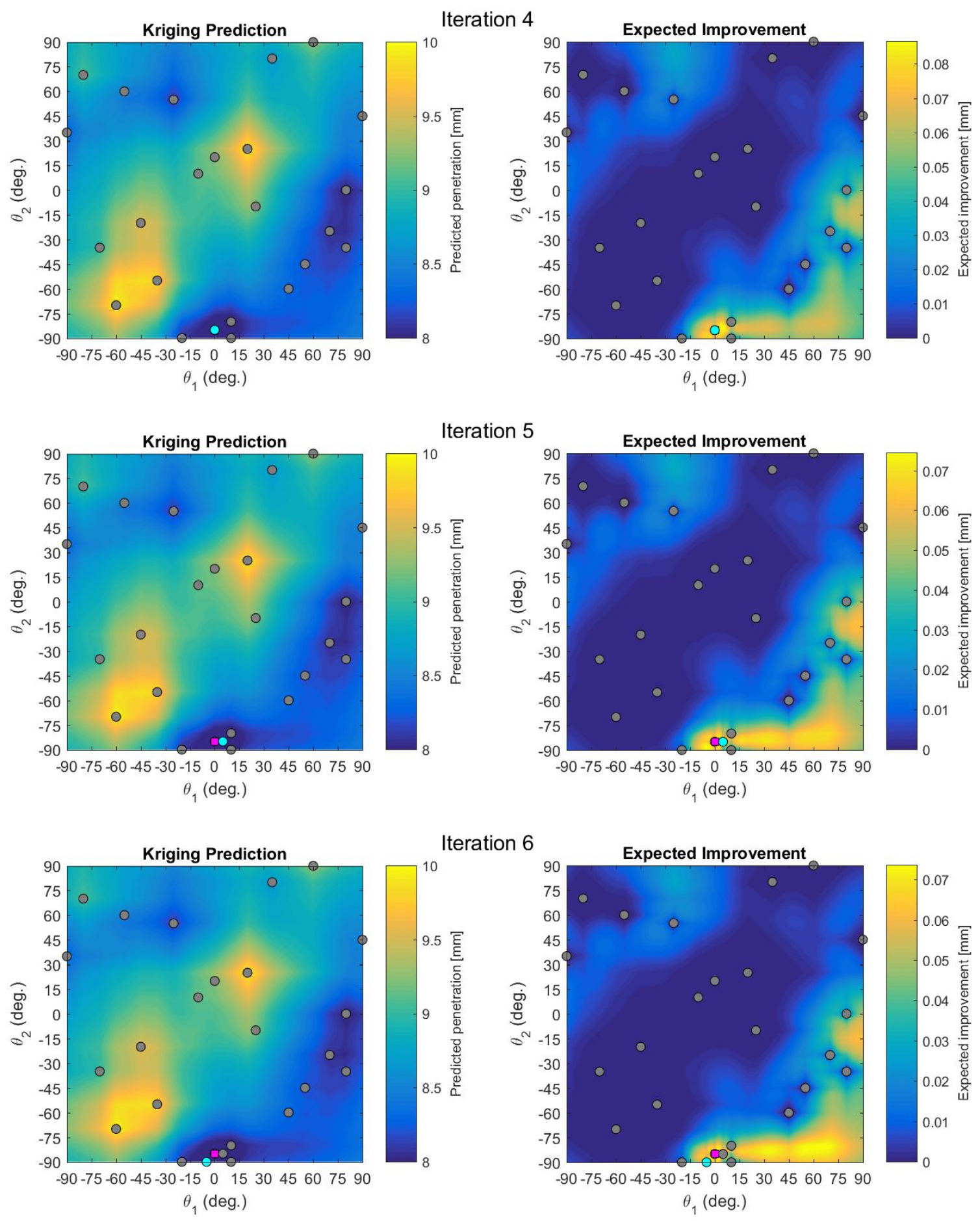

Figure 4.4. EGO optimization progress - Armor problem, symmetric impact. Current sample (grey), best current design (purple), last sampled design (cyan). Iterations 4,5 and 6. 


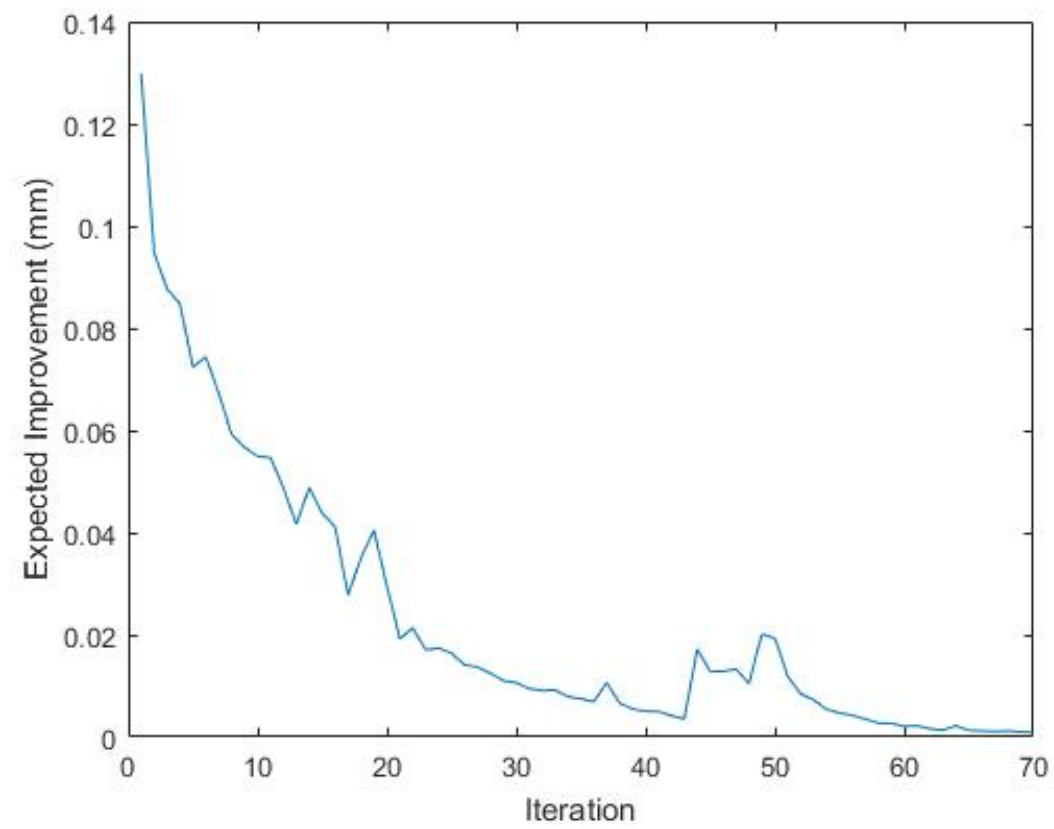

Figure 4.5. Armor plate problem - symmetric impact. Expected improvement at each iteration.

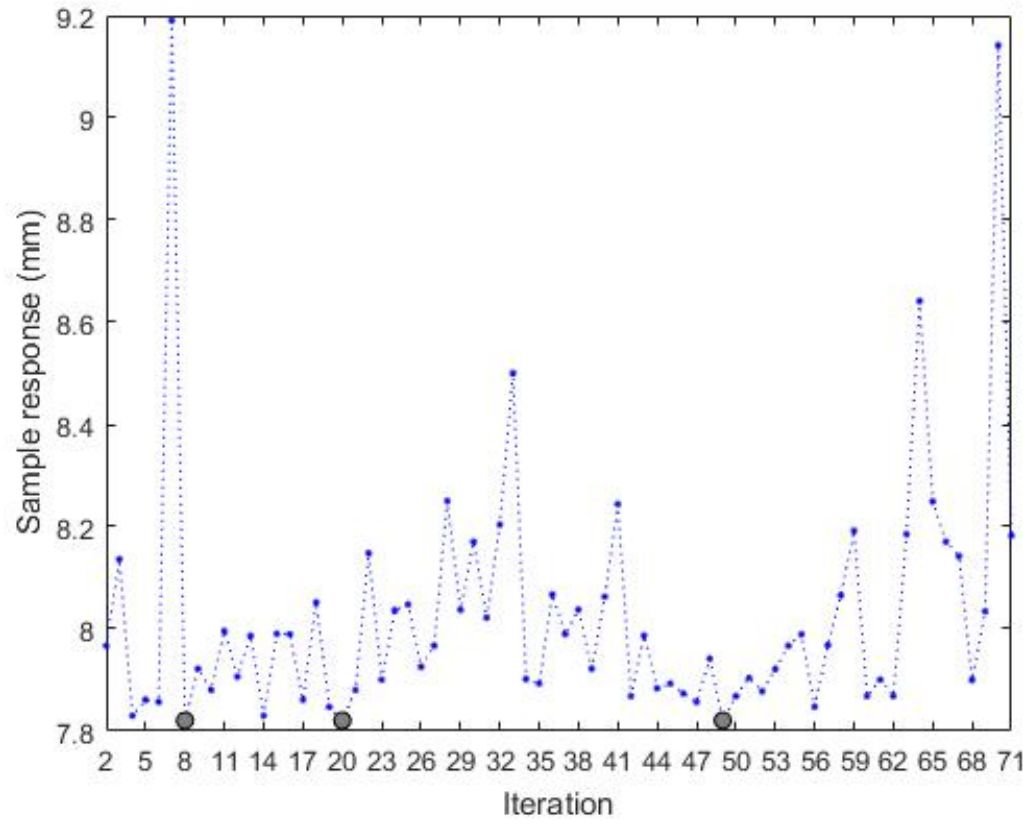

Figure 4.6. Armor plate problem - symmetric impact. Response of the designs sampled by EGO at each iteration. Optimal responses (gray dots). 

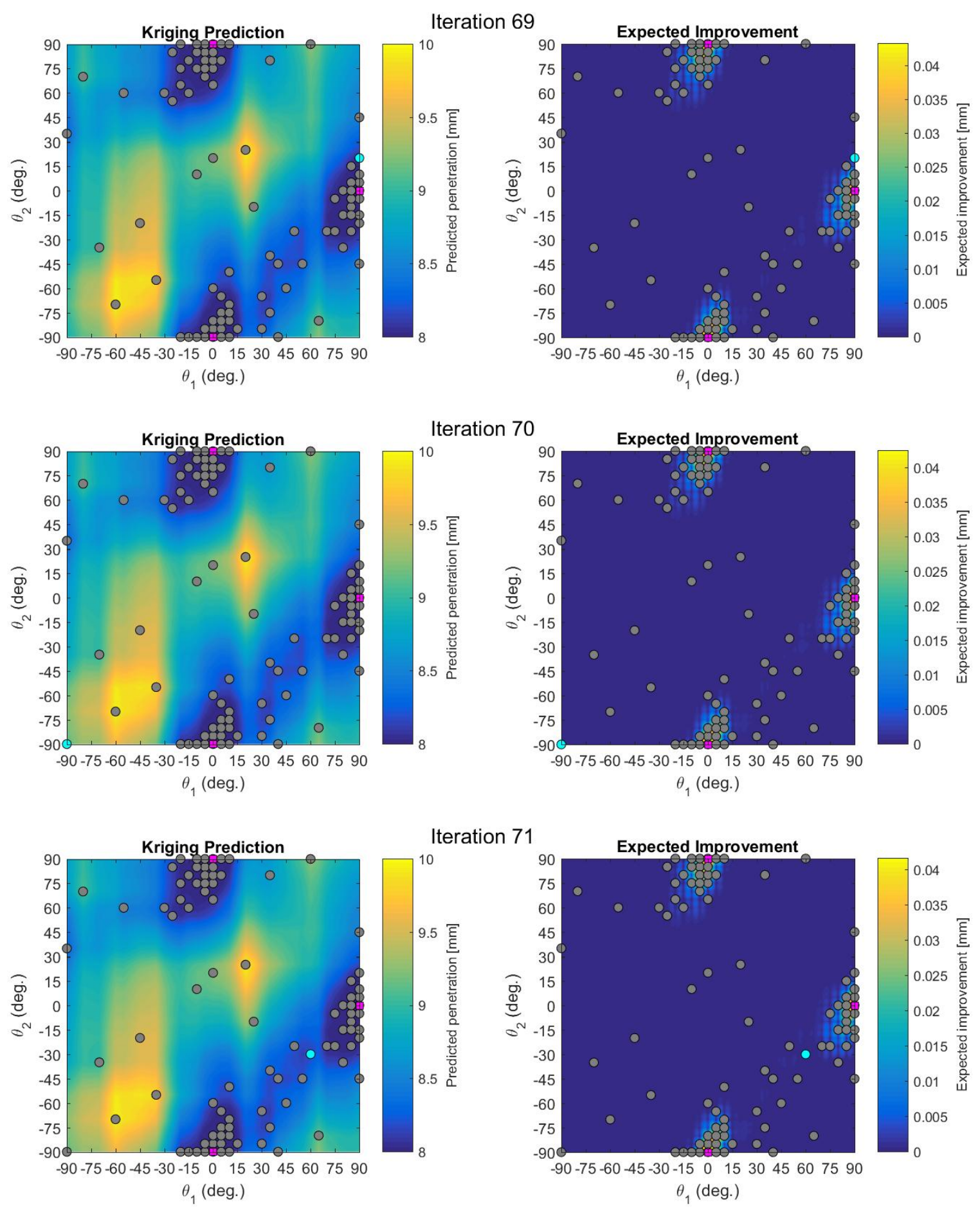

Figure 4.7. EGO optimization progress - Armor problem, symmetric impact. Current sample (grey), best current design (purple), last sampled design (cyan). Iterations 69, 70 and 71. 


\subsubsection{Non-Symmetric Impact}

This example is proposed because of its difficulty to find intuitive solutions. To obtain the non-symmetric conditions, the sphere impacts the armor with an offset of $50 \mathrm{~mm}$ and $70 \mathrm{~mm}$ from one vertex of the armor (Fig. 4.8).

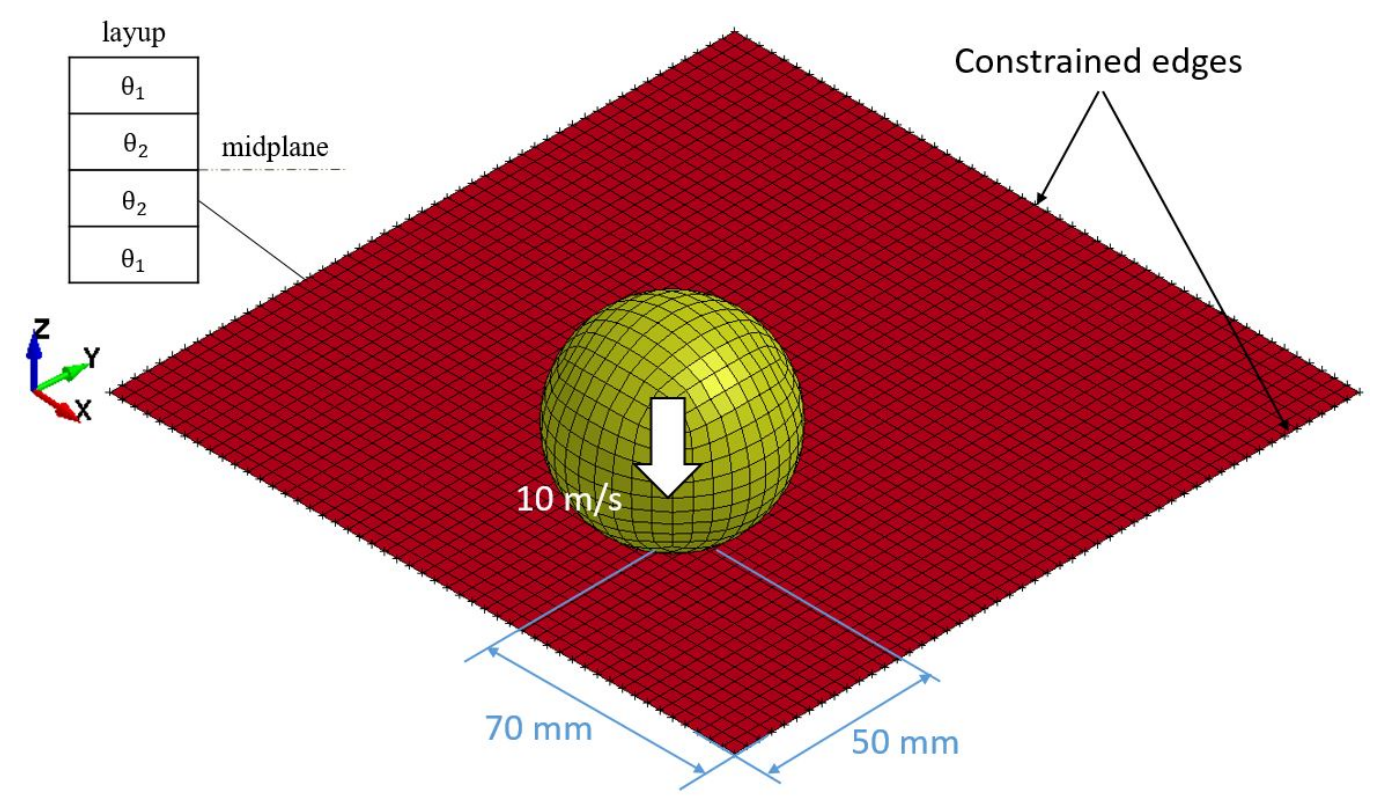

Figure 4.8. Armor plate problem - non-symmetric impact

The same sample designs of Table 4.4 are used to generate the metamodels to be validated. The best surrogate for the armor's penetration has a second-order regression function and an exponential correlation (Table 4.7). This surrogate has a

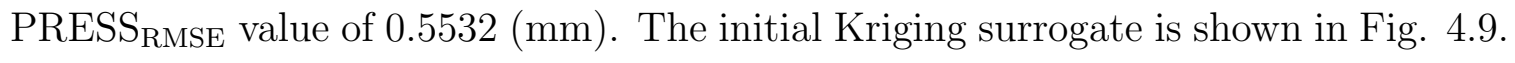

Table 4.7.

Armor plate problem - non-symmetric impact. PRESS RMSE $_{\text {of Kriging }}$ surrogates using different regression and correlation functions.

\begin{tabular}{|ccc|ccc|}
\hline \multicolumn{2}{|c|}{ Exponential correlation } & \multicolumn{3}{c|}{ Gaussian correlation } \\
\hline regpoly0 & regpoly1 & regpoly2 & regpoly0 & regpoly1 & regpoly2 \\
0.6651 & 0.7294 & 0.5532 & 0.5696 & 0.6287 & 0.6208 \\
\hline
\end{tabular}




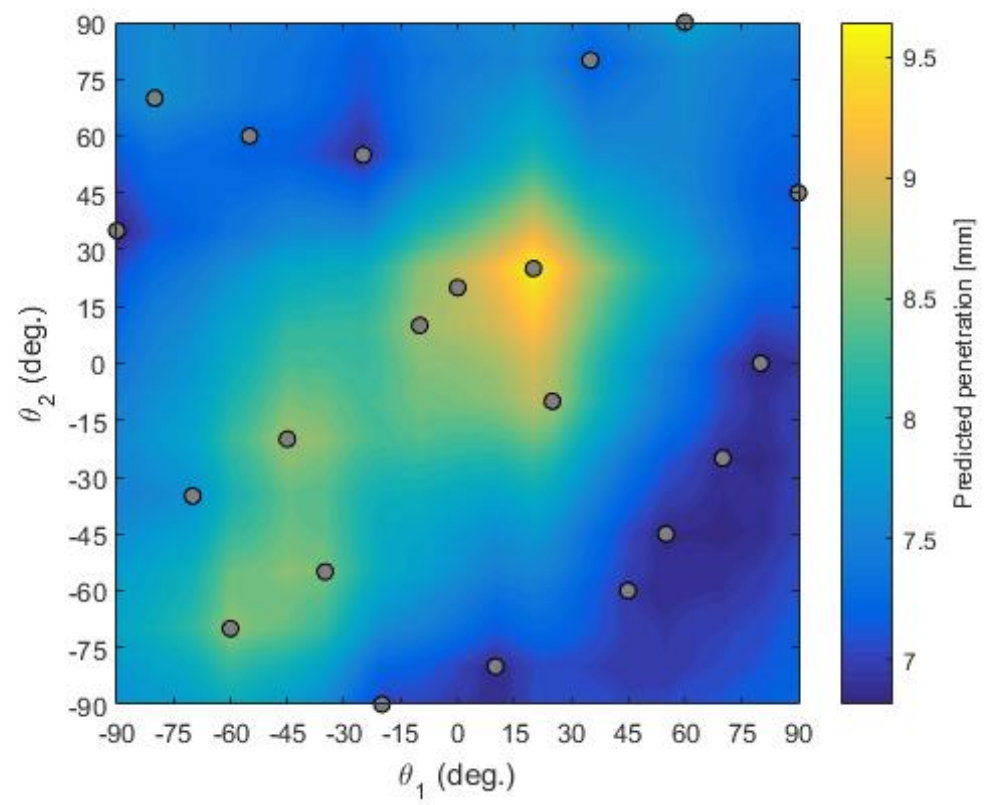

Figure 4.9. Armor problem, non-symmetric impact. Initial Kriging surrogate of the armor's penetration based on the 21 LHS sampled points shown as dots.

Fig. 4.10 and 4.11 illustrate the first six-EGO iterations. They show that the areas with high expected improvement values are sampled at the beginning of the optimization process. In comparison with the symmetric impact, EGO needs more iterations to find the optimal design under non-symmetric loading. The optimal solution is found at iteration 67 (Fig. 4.13). The optimal layup is $\left[-85^{\circ} / 35^{\circ}\right]_{s}$. This design has a maximum penetration of $6.6014 \mathrm{~mm}$.

As in the symmetric loading case, the expected improvement of the surrogate approaches to zero along the optimization process. The convergence criteria is satisfied at iteration 104 (Fig. 4.12).

Fig. 4.13 contains the responses of the samples added by EGO. Their values reflect that the metamodel is exploited and that the design space is explored along the optimization process. EGO selects designs that lead to EI maximization but meeting the constraint of degree increments as shown in Fig. 4.14. 

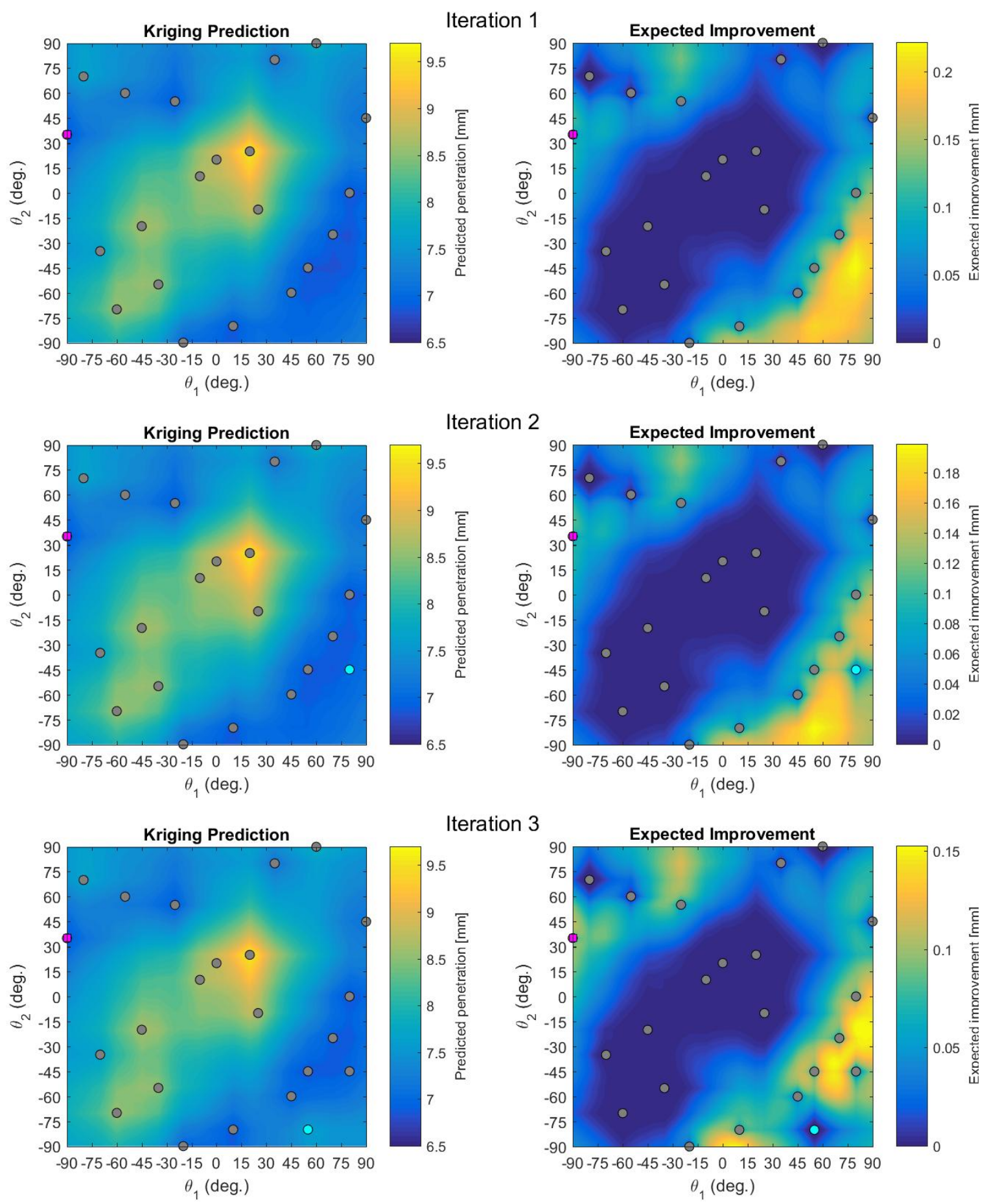

Figure 4.10. EGO optimization progress - Armor problem, nonsymmetric impact. Current sample (grey), best current design (purple), last sampled design (cyan). Iterations 1, 2 and 3. 

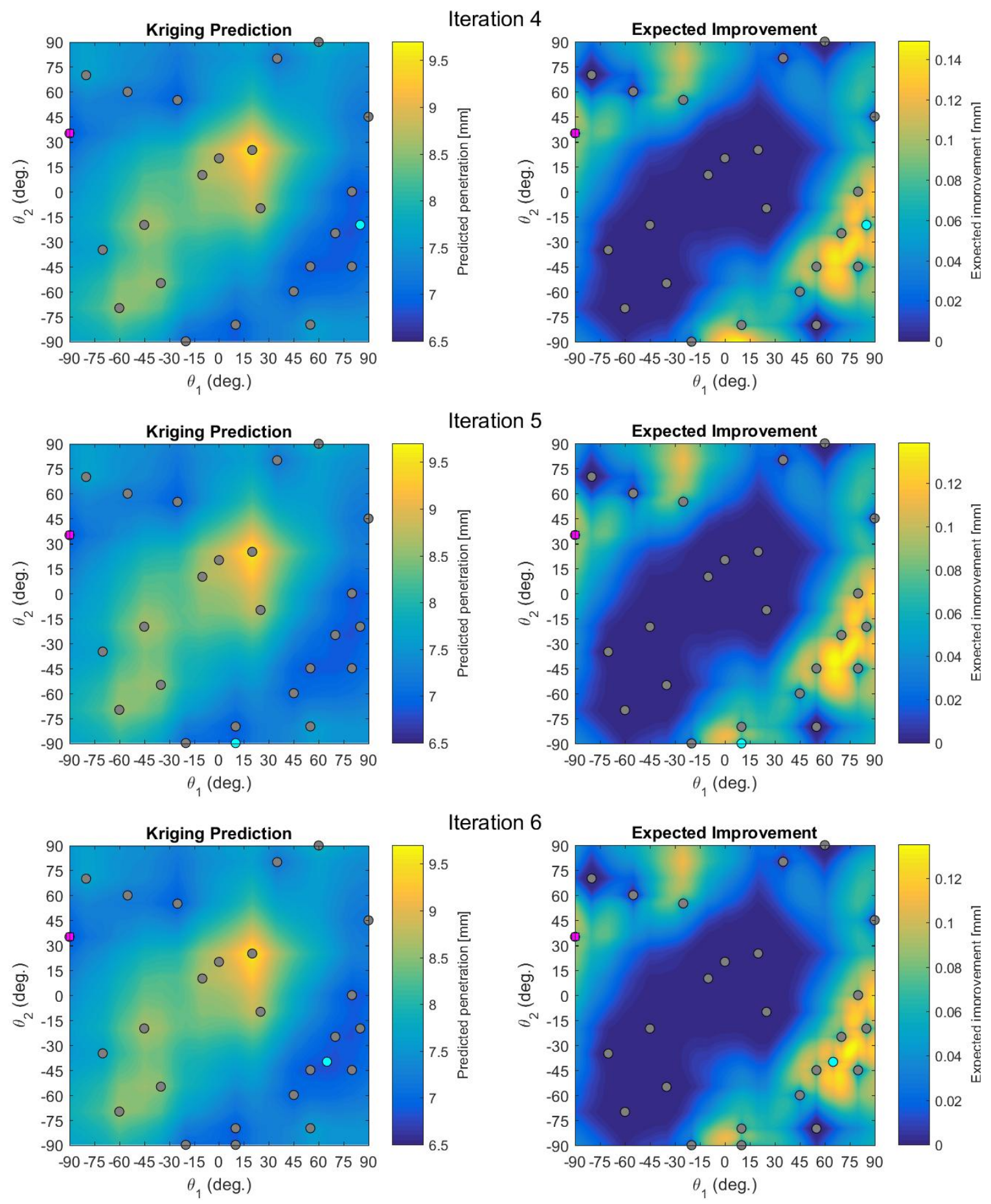

Figure 4.11. EGO optimization progress - Armor problem, nonsymmetric impact. Current sample (grey), best current design (purple), last sampled design (cyan). Iterations 4,5 and 6 . 


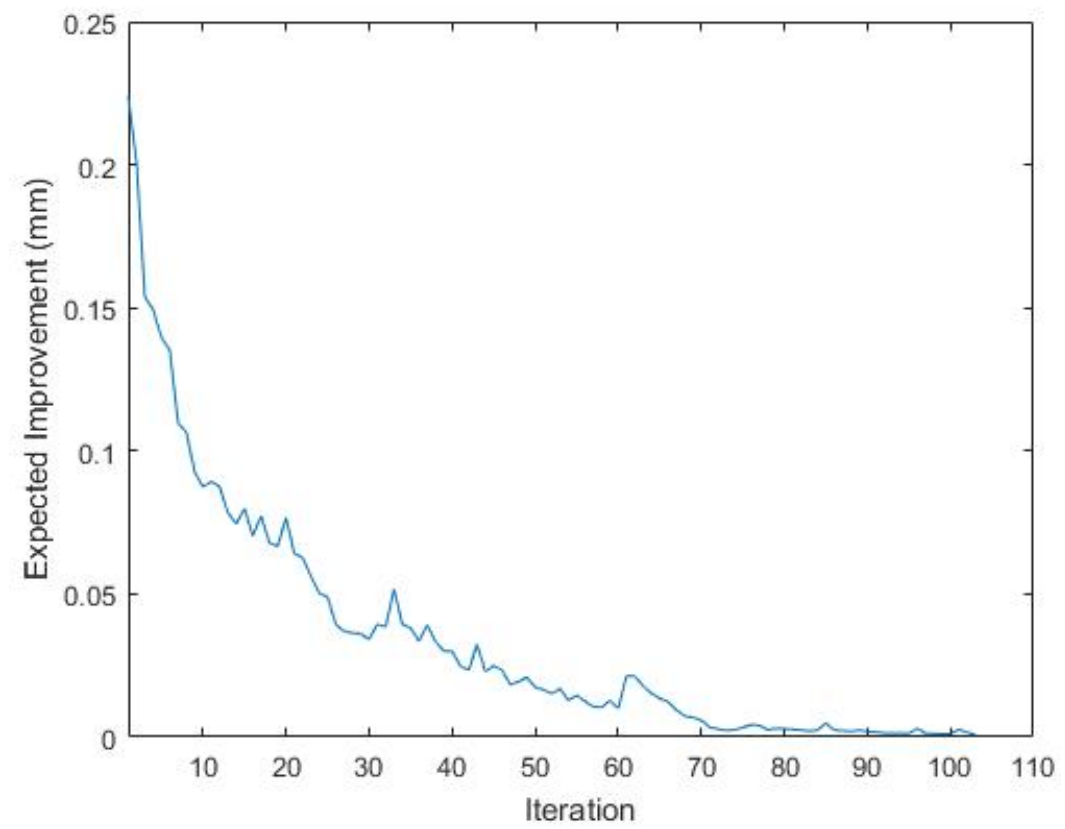

Figure 4.12. Armor plate problem - non-symmetric impact. Expected improvement at each iteration.

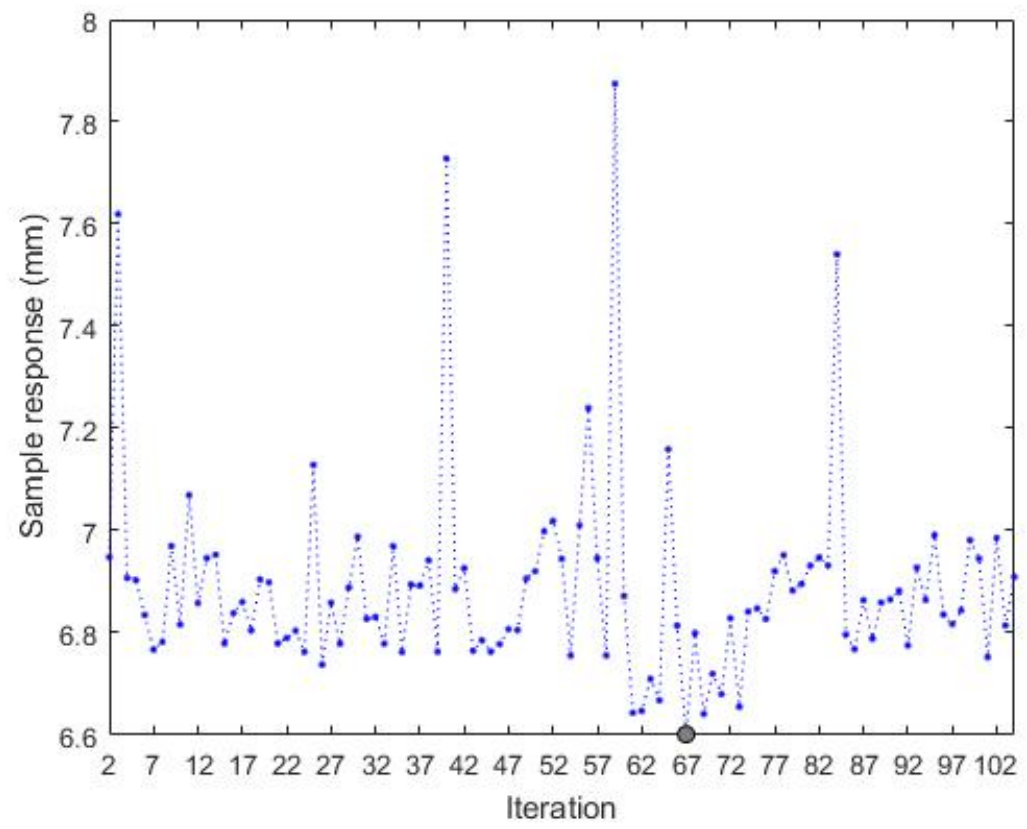

Figure 4.13. Armor plate problem - non-symmetric impact. Response of the designs sampled by EGO at each iteration. Optimal response (gray dot). 


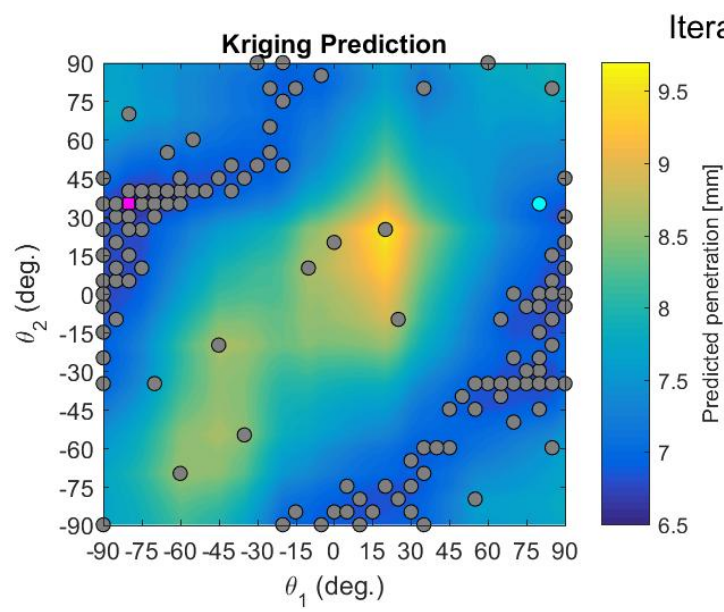

Iteration $102 \quad$ Expected Improvement
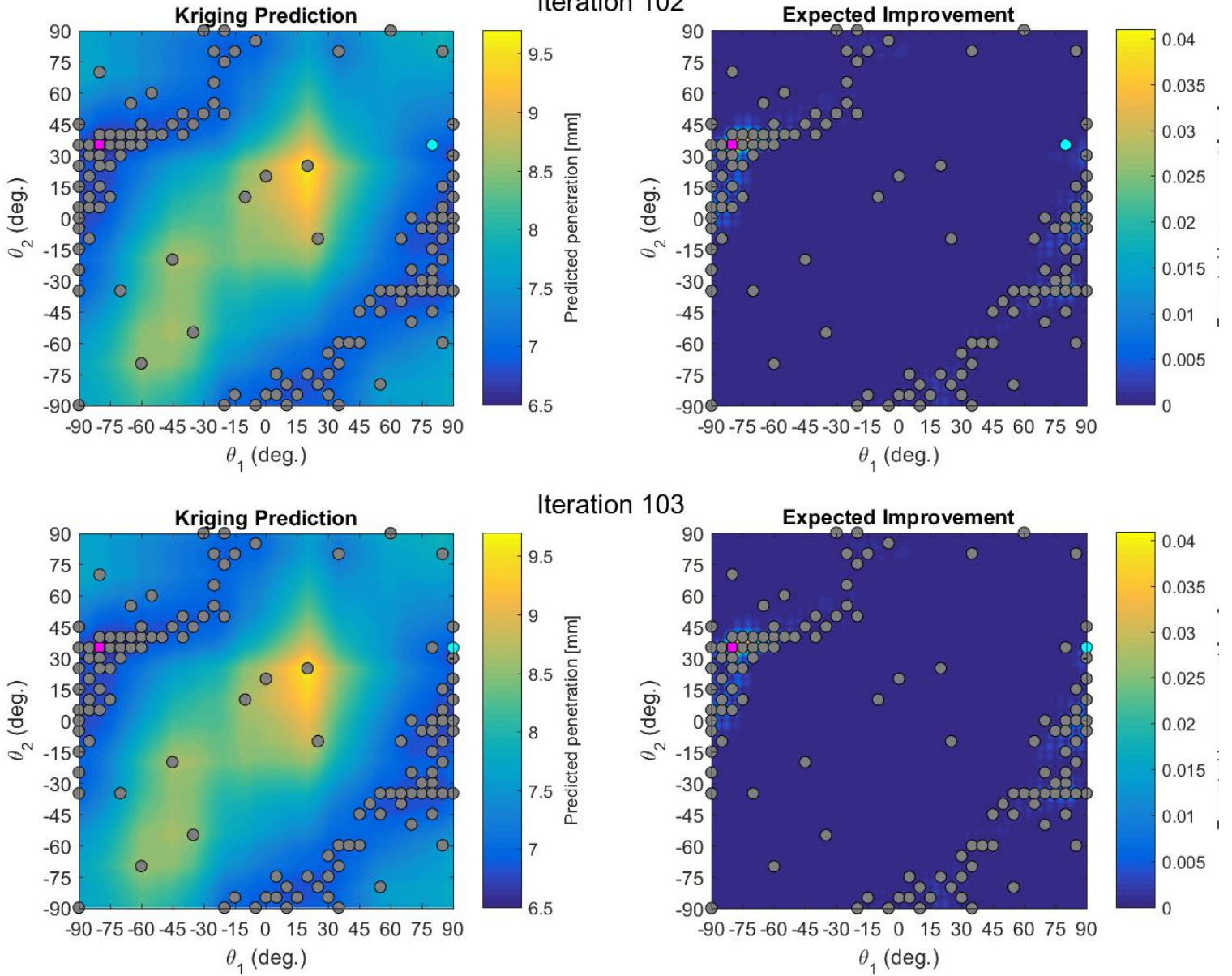

Iteration 103
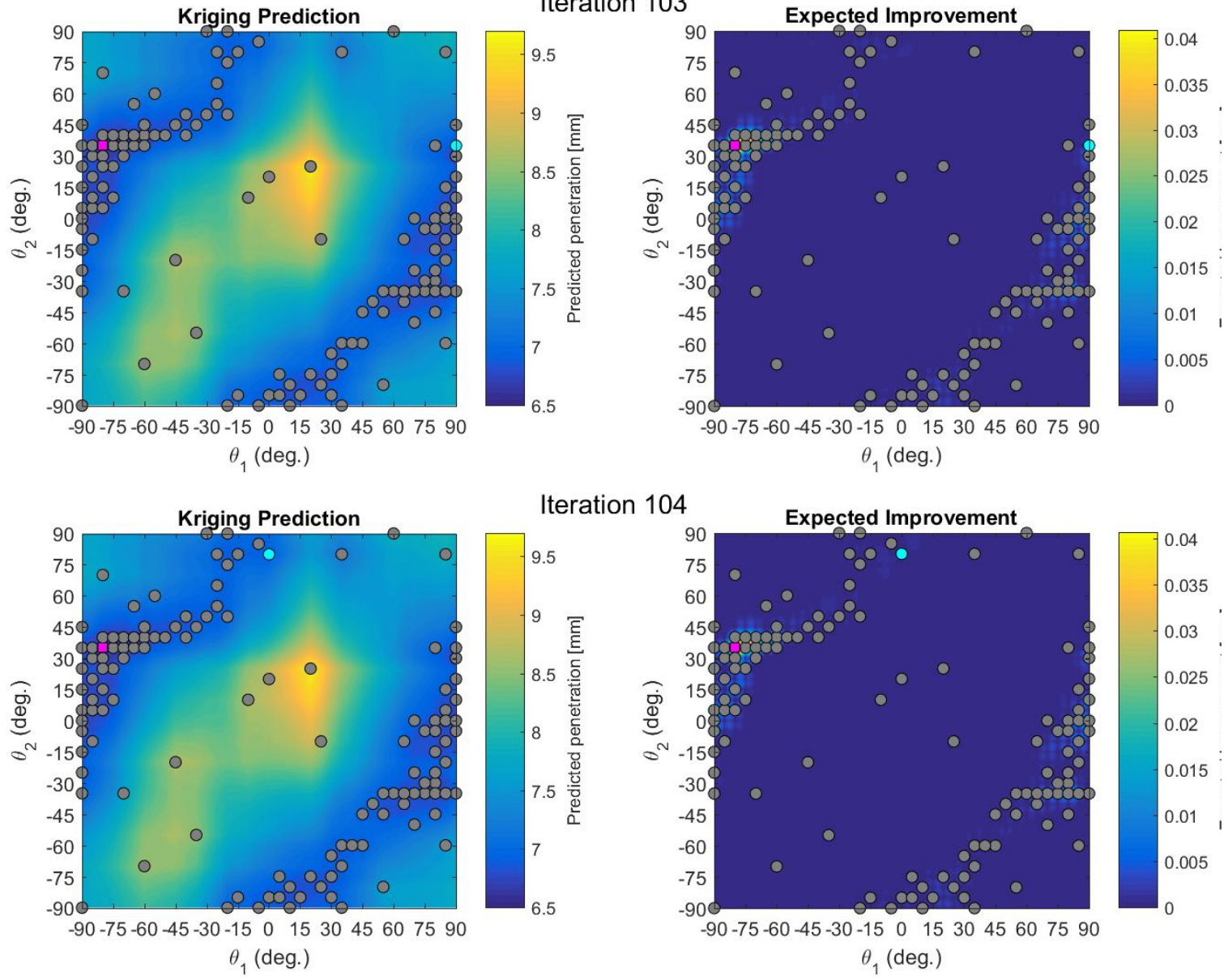

Iteration 104

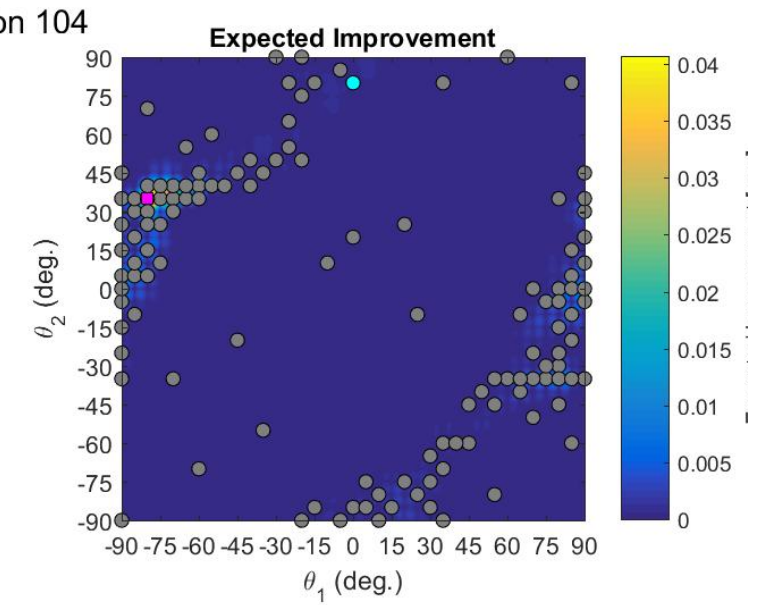

Figure 4.14. EGO optimization progress - Armor problem, nonsymmetric impact. Current sample (grey), best current design (purple), last sampled design (cyan). Iterations 102, 103 and 104. 


\subsection{Minimum Displacement and Acceleration under Dynamic Load on a Composite Tube}

In the following examples, the composite tube is impacted by a rigid wall. Two loading cases are studied, axial and oblique impact. In order to control the collapse of the tube, it is divided in four sections. The sections are made of 4, 6, 8 and 10 plies that form angle-ply laminates, $[ \pm \theta]_{n}$, e.g., a $\left[45^{\circ} /-45^{\circ} / 45^{\circ}-45^{\circ}\right]$ laminate is a possible design for the section with 4 plies. Each ply has a thickness of $0.5 \mathrm{~mm}$. For the ease of manufacturing the orientations are restricted to $\left\{0^{\circ}, 15^{\circ}, 30^{\circ}, 45^{\circ}, 60^{\circ}, 75^{\circ}, 90^{\circ}\right\}$.

The composite tube has an square section of $100 \times 100 \mathrm{~mm}$ and a length of $1 \mathrm{~m}$. One end of the tube is fixed. The rigid wall has a mass of $1000 \mathrm{~kg}$ and an initial velocity of $2.5 \mathrm{~m} / \mathrm{s}$.

For the numerical analysis, the composite tube has 4000 identical finite elements of size $10 \times 10 \mathrm{~mm}$. The convergence criteria to terminate the optimization is an expected improvement less than $1 \times 10^{-3}$. The maximum number of iterations is 150 .

The optimization algorithm finds the lengths of each section of the tube and the fiber orientation that minimize the displacement of the rigid wall and its peak acceleration during the impact. The formulation of the optimization problem is,

$$
\begin{array}{ll}
\text { find } & \mathbf{x} \in \mathbb{R}^{4} \\
\text { minimize } & f_{1}(\mathbf{x}): \text { maximum displacement of the wall } \\
\text { minimize } & f_{2}(\mathbf{x}): \text { peak acceleration of the wall } \\
\text { subject to } & 0 \leq x_{i} \leq 150 \\
& \theta \in\left\{0^{\circ}, 15^{\circ}, 30^{\circ}, 45^{\circ}, 60^{\circ}, 75^{\circ}, 90^{\circ}\right\}
\end{array}
$$

where $\mathbf{x}=\left[\begin{array}{lll}x_{1} & x_{2} & x_{3}\end{array}\right], x_{i}$ are the lengths of the first three tube sections and $\theta$ is the fiber orientation that define the angle-ply laminates. 


\subsubsection{Axial Impact}

In this example, the rigid wall impacts the composite tube axially as illustrated in Fig. 4.15. The 43 LHS samples of Table 4.8 are used to create the initial metamodels for the displacement and acceleration of the wall $\left(f_{1}(\mathbf{x})\right.$ and $\left.f_{2}(\mathbf{x})\right)$. Fig. 4.16 shows the distribution of the samples in the design space.

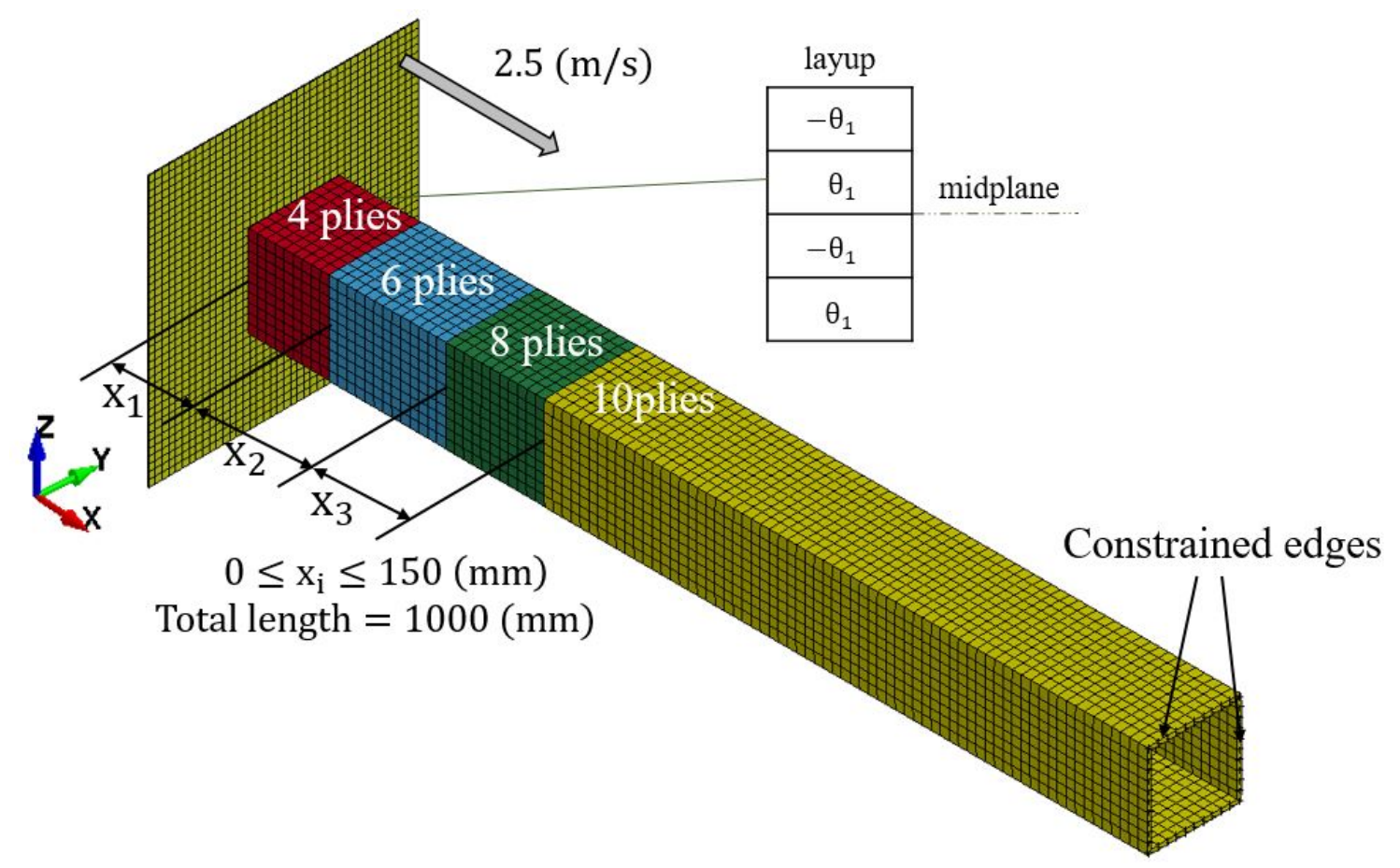

Figure 4.15. Composite tube problem - axial impact.

According to Table 4.9, the best surrogate for the displacement has a first-order regression function and exponential correlation. In the case of the acceleration, a zeroorder regression function and exponential correlation leads to the lowest PRESS $\mathrm{RMSE}_{\mathrm{B}}$ (Table 4.10).

Fig. 4.17 shows the responses of the 43 LHS samples. At each iteration, EGO maximizes the multi-objective expected improvement function in order to find designs that improve (expand) the current Pareto front. Fig. 4.18 contains the first four EGO iterations. The first expansion of the Pareto front occurs at iteration 3. 
Table 4.8.

LHS sampling - Composite tube problem

\begin{tabular}{|ccc|c|ccc|c|ccc|c|}
\hline $\mathrm{x}_{1}$ & $\mathrm{x}_{2}$ & $\mathrm{x}_{3}$ & $\theta$ & $\mathrm{x}_{1}$ & $\mathrm{x}_{2}$ & $\mathrm{x}_{3}$ & $\theta$ & $\mathrm{x}_{1}$ & $\mathrm{x}_{2}$ & $\mathrm{x}_{3}$ & $\theta$ \\
\hline & $(\mathrm{mm})$ & & $(\mathrm{deg})$, & & $(\mathrm{mm})$ & & $($ deg. $)$ & & $(\mathrm{mm})$ & & $($ deg. $)$ \\
\hline 120 & 90 & 110 & 60 & 70 & 50 & 40 & 60 & 20 & 120 & 140 & 30 \\
100 & 30 & 140 & 75 & 20 & 140 & 80 & 30 & 0 & 10 & 130 & 0 \\
100 & 40 & 20 & 15 & 100 & 40 & 130 & 90 & 10 & 50 & 100 & 75 \\
120 & 100 & 30 & 45 & 140 & 140 & 90 & 90 & 80 & 110 & 90 & 60 \\
60 & 70 & 10 & 75 & 20 & 60 & 20 & 75 & 130 & 120 & 50 & 75 \\
80 & 50 & 70 & 90 & 130 & 60 & 60 & 15 & 40 & 20 & 140 & 15 \\
80 & 90 & 40 & 45 & 10 & 20 & 10 & 30 & 60 & 30 & 20 & 60 \\
50 & 120 & 130 & 0 & 90 & 10 & 80 & 0 & 140 & 100 & 100 & 45 \\
50 & 80 & 10 & 45 & 110 & 40 & 60 & 60 & 130 & 70 & 90 & 0 \\
40 & 80 & 0 & 0 & 60 & 110 & 150 & 75 & 90 & 90 & 120 & 30 \\
50 & 80 & 80 & 15 & 110 & 130 & 110 & 90 & 140 & 30 & 60 & 15 \\
30 & 60 & 110 & 45 & 120 & 100 & 70 & 30 & 90 & 20 & 100 & 60 \\
110 & 10 & 40 & 90 & 70 & 0 & 120 & 45 & 40 & 130 & 120 & 15 \\
150 & 110 & 30 & 60 & 30 & 130 & 30 & 0 & & & & \\
10 & 150 & 50 & 30 & 30 & 140 & 50 & 90 & & & & \\
\hline
\end{tabular}

Table 4.9 .

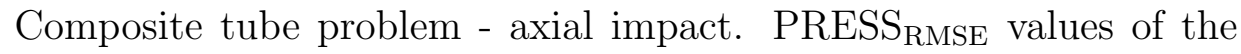
Kriging metamodels for the wall displacement.

\begin{tabular}{|ccc|ccc|}
\hline \multicolumn{2}{|c|}{ Exponential correlation } & \multicolumn{4}{c|}{ Gaussian correlation } \\
\hline regpoly0 & regpoly1 & regpoly2 & regpoly0 & regpoly1 & regpoly2 \\
47.9857 & 45.5177 & 143.282 & 59.6901 & 64.9324 & 154.2355 \\
\hline
\end{tabular}

Due to the different units of the objective functions, the multi-objective expected improvement is normalized to have a dimensionless value from zero to one. To obtain 
Table 4.10.

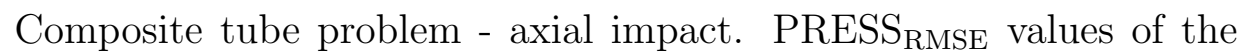
Kriging meatmodels for the peak acceleration of the wall.

\begin{tabular}{|ccc|ccc|}
\hline \multicolumn{2}{|c|}{ Exponential correlation } & \multicolumn{3}{c|}{ Gaussian correlation } \\
\hline regpoly0 & regpoly1 & regpoly2 & regpoly0 & regpoly1 & regpoly2 \\
$6.23 \mathrm{E}+04$ & $1.01 \mathrm{E}+05$ & $1.67 \mathrm{E}+05$ & $9.23 \mathrm{E}+04$ & $9.78 \mathrm{E}+04$ & $1.49 \mathrm{E}+05$ \\
\hline
\end{tabular}
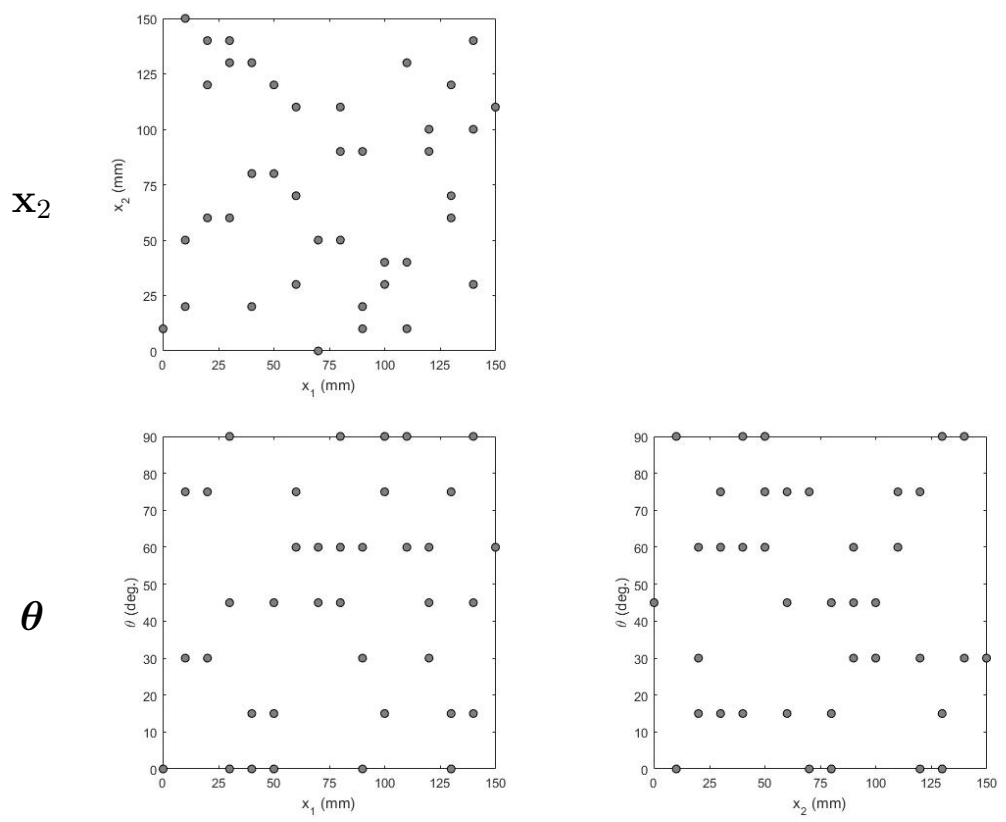

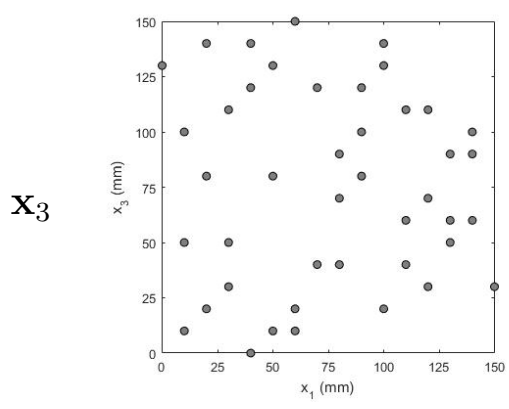

$\mathbf{X}_{1}$

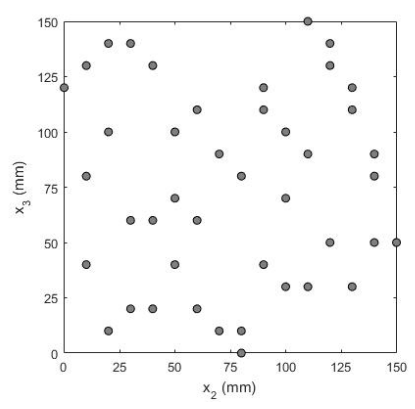

$\mathbf{x}_{2}$

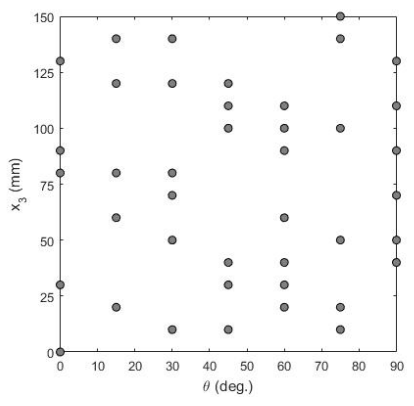

$\theta$

Figure 4.16. Composite tube problem. Sampling over design space using 43 LHS designs. 


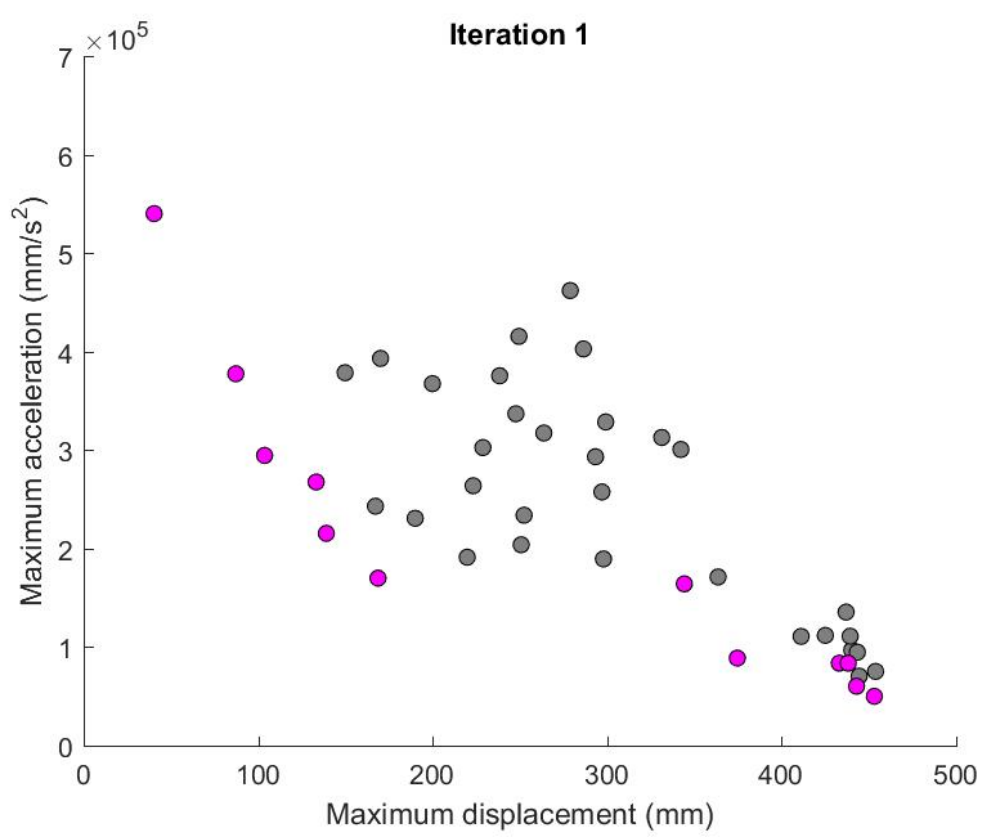

Figure 4.17. Composite tube problem - axial impact. Initial responses for the 43 LHS samples. LHS samples (grey), Pareto front (purple).

this dimensionless value, the distance between the centroid of the probability integral $(\overline{\mathrm{F}} 1(x), \overline{\mathrm{F}} 2(x))$ and the closest Pareto design $\left(\left(y 1^{*}, y 2^{*}\right)\right)$ is scaled to a value between 0 and 1 . The normalized expected improvement is defined as,

$$
\bar{E}\left[I\left(x^{*(p)}\right)\right]=P\left[I\left(x^{*(p)}\right)\right] \min \left(\bar{d}_{1}, \ldots, \overline{d_{m}}\right)
$$

Fig. 4.19 shows the responses of the samples at iteration 150. Although the algorithm tends to obtain evenly spaced designs along the Pareto front, there is a large number of designs that present low accelerations and large displacements. Fig. 4.20 is a comparison between the initial and final Pareto Fronts. EGO has improved the Pareto front by replacing almost all the initial designs. Table 4.11 contains the new optimal designs.

Regarding the algorithm convergence, Fig. 4.21 shows that the expected improvement decreases along the optimization. EGO requires more iterations to satisfy the stopping criterion. 

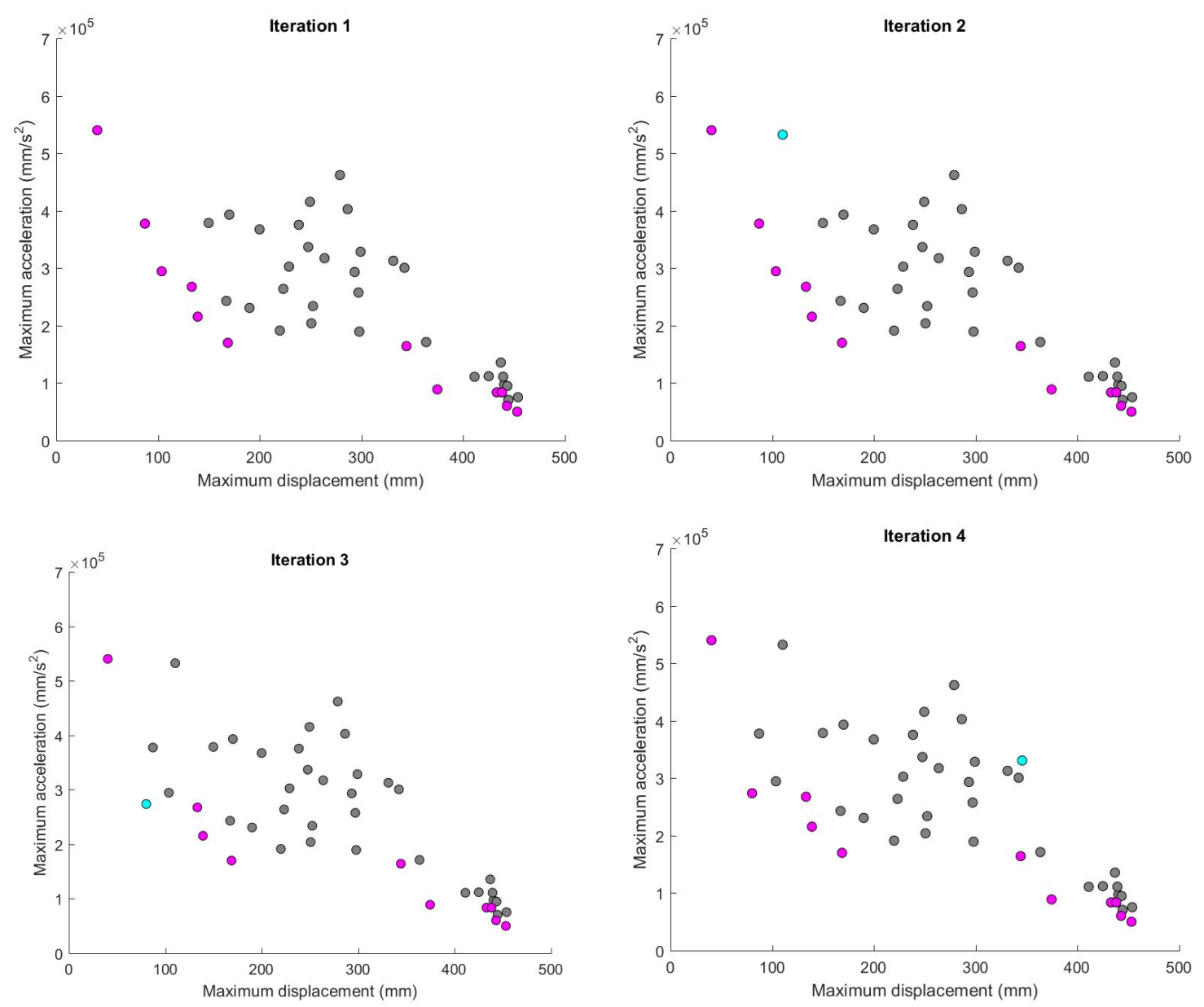

Figure 4.18. EGO optimization progress - Composite tube problem, axial impact. Dominated designs (grey), Pareto front (purple), last sampled design (cyan). Iterations 1, 2, 3 and 4.

It is observed than some designs of the final Pareto front are non-feasible. The supports of the designs whose displacements are larger than $200 \mathrm{~mm}$ fail during the impact. This failure allows large displacements and low accelerations that EGO misinterprets as optimal solutions. The zone of non-feasible designs is shown in Fig. 4.22 .

To avoid the sampling of non-feasible designs, the wall is limited to have displacements less or equal to $200 \mathrm{~mm}$. The new constrained optimization problem is defined as, 


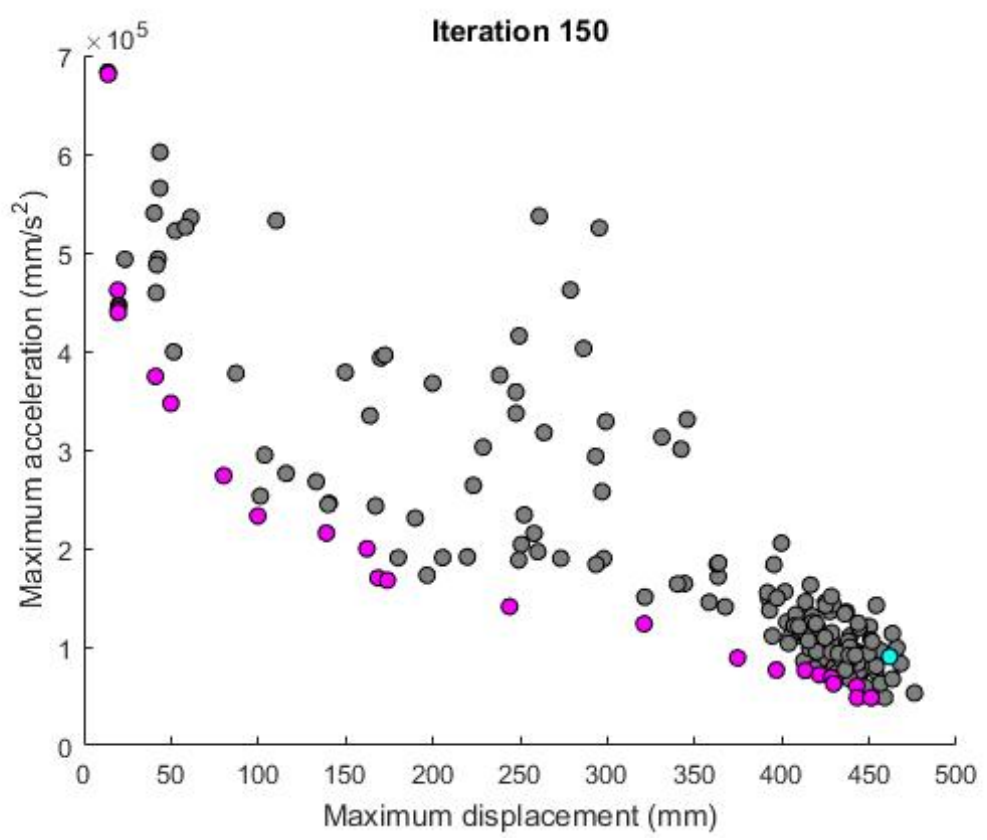

Figure 4.19. Composite tube problem - axial impact. Responses at the last iteration. Dominated designs (grey), Pareto front (purple).

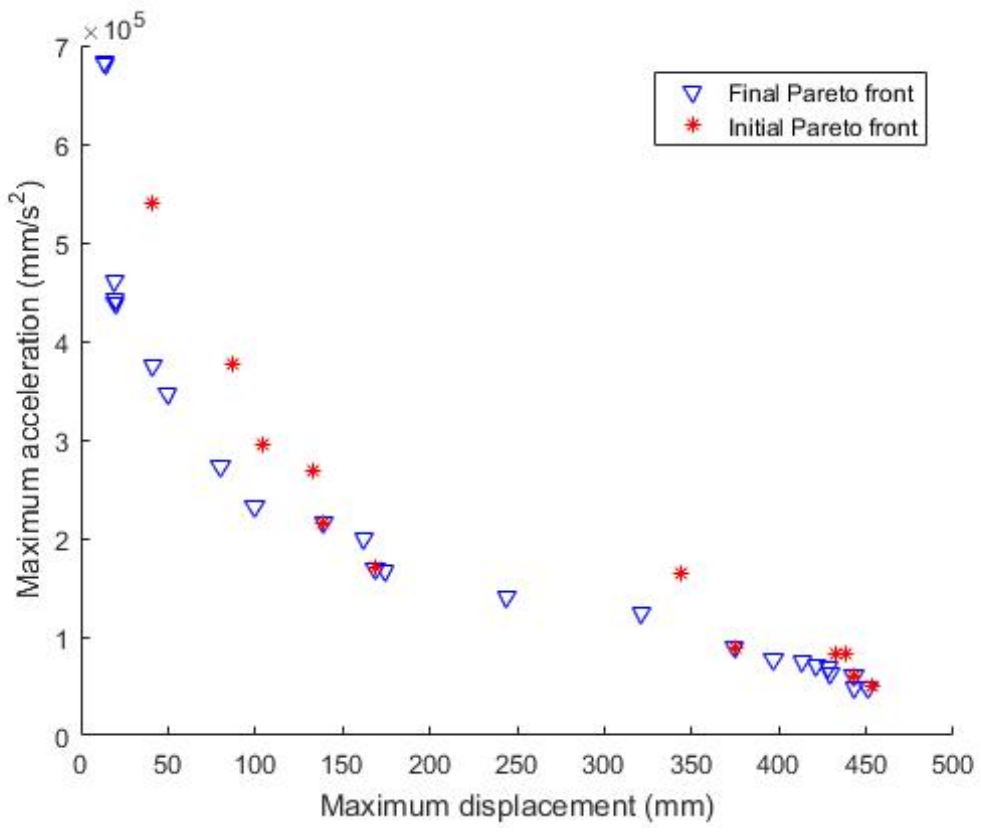

Figure 4.20. Composite tube problem - axial impact. Initial and final Pareto fronts. 


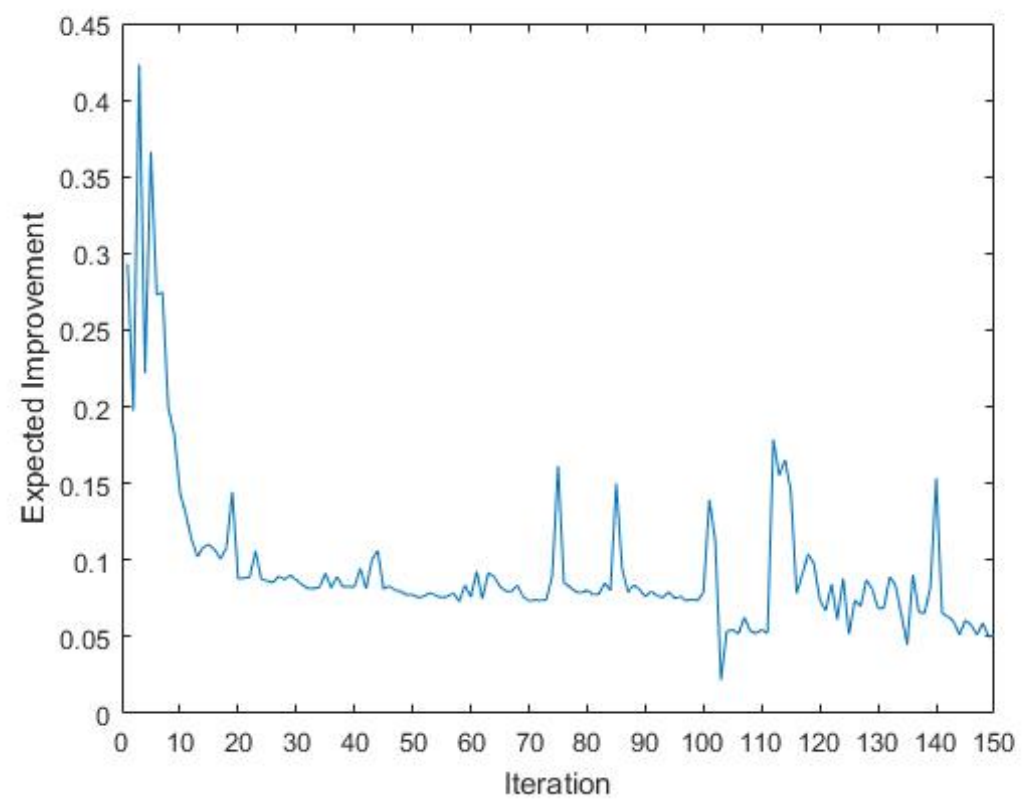

Figure 4.21. Composite tube problem - axial impact. Expected improvement at each iteration.

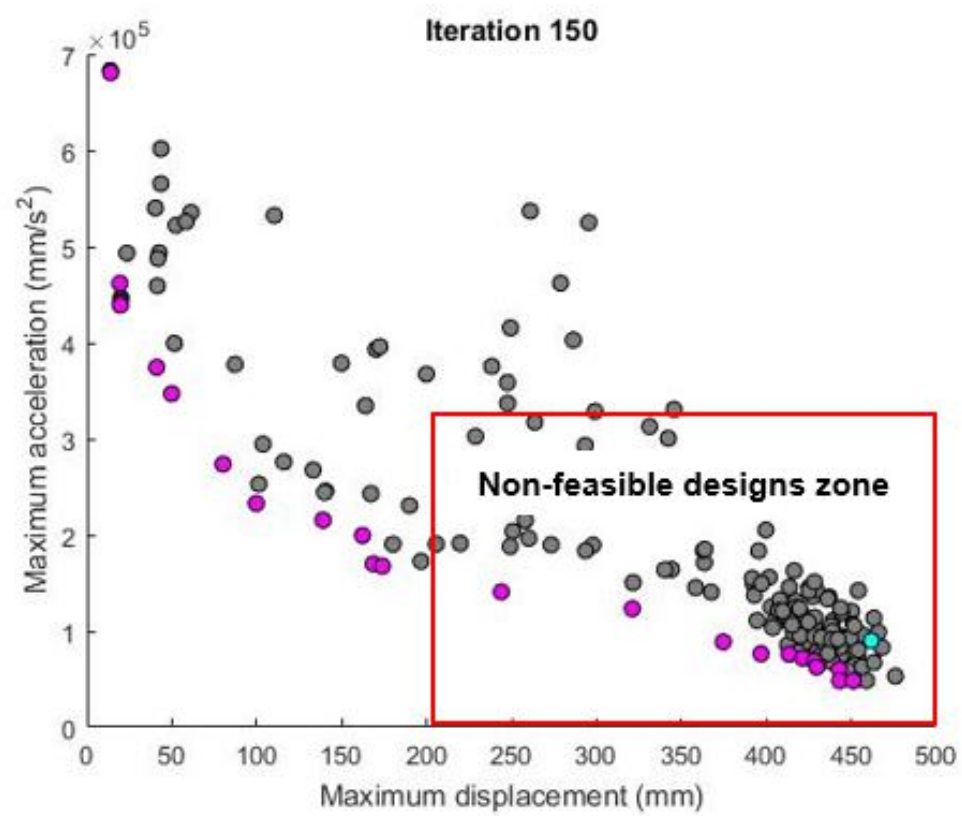

Figure 4.22. Composite tube problem - axial impact. Non-feasible designs in the final Pareto front. 


$$
\begin{array}{ll}
\text { find } & \mathbf{x} \in \mathbb{R}^{4} \\
\text { minimize } & f_{1}(\mathbf{x}): \text { maximum displacement of the wall } \\
\text { minimize } & f_{2}(\mathbf{x}): \text { peak acceleration of the wall } \\
\text { subject to } & f_{1}(\mathbf{x}) \leq 200 \\
& 0 \leq x_{i} \leq 150 \\
& \theta \in\left\{0^{\circ}, 15^{\circ}, 30^{\circ}, 45^{\circ}, 60^{\circ}, 75^{\circ}, 90^{\circ}\right\}
\end{array}
$$

The MEI function is modified to include the constraint effect in the optimization process. A metamodel for the constraint is created when its functional form is unknown. Then, the probability that a predicting point $x$ satisfies the constraint is included in the EI formulation.

$$
E[I(x) \cap G(x)]=E[I(x)] P[G(x)]
$$

Where $E[I(x) \cap G(x)]$ is the constrained expected improvement and $P[G(x)]$ is the probability that the constraint $G(x)$ is satisfied at the predicting point $x$.

The probability of constraint satisfaction is calculated as,

$$
P[G(x)]=\frac{1}{2}\left[1+\operatorname{erf}\left(\frac{g_{\max }-\hat{g}(x)}{\sqrt{2} \hat{s}(x)}\right)\right]
$$

where $g_{\max }$ is the constrain limit (200 $\mathrm{mm}$ in this example) $\hat{g}(x)$ and $\hat{s}(x)$ are the prediction and variance provided by the Kriging surrogate of the constraint.

Fig. 4.23 shows the samples responses at the last iteration using the constrained MEI. The constraint stimulates the sampling before the limit value $(200 \mathrm{~mm})$. More feasible optimal designs are present in the final Pareto front (Table 4.12).

In Fig. 4.24 the final Pareto fronts using constrained and unconstrained EI are shown. The constrained EI provides two advantages. It generates a broader expansion of the Pareto front and the predictions of the metamodels are more accurate in the feasible zone than the unconstrained formulation. 


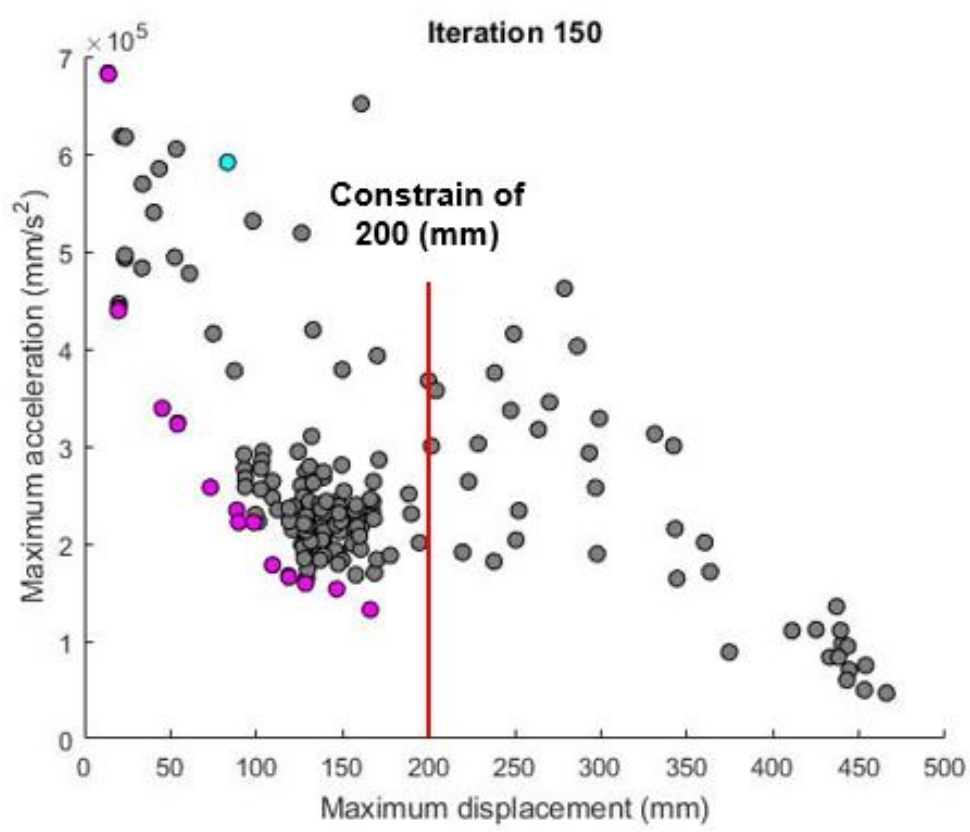

Figure 4.23. Composite tube problem - axial impact. Responses at the last iteration using constrained improvement formulation.

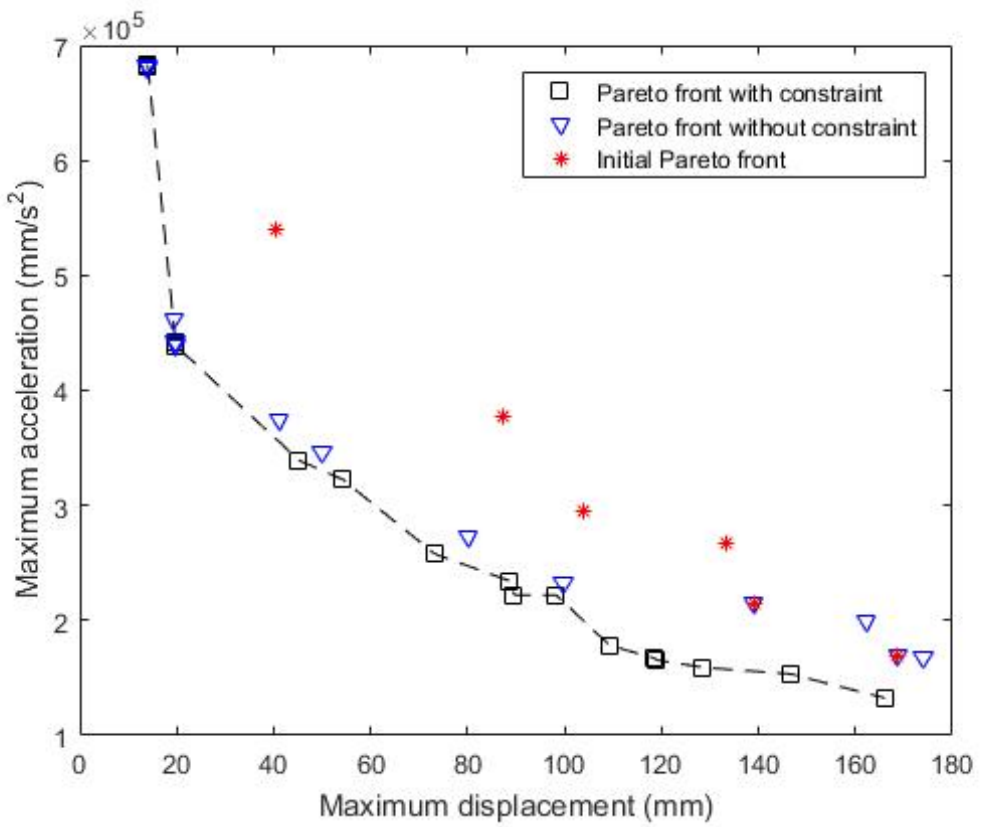

Figure 4.24. Composite tube problem - axial impact. Comparison of Pareto fronts using constrained and unconstrained expected improvement. 
Table 4.11.

Composite tube problem - axial impact. Optimal designs and responses

\begin{tabular}{|c|c|c|c|c|c|}
\hline $\begin{array}{c}\mathrm{x}_{1} \\
(\mathrm{~mm})\end{array}$ & $\begin{array}{c}\mathrm{x}_{2} \\
(\mathrm{~mm})\end{array}$ & $\begin{array}{c}\mathrm{x}_{3} \\
(\mathrm{~mm})\end{array}$ & $\begin{array}{c}\theta \\
\text { (deg.) }\end{array}$ & $\begin{array}{c}\text { Maximum } \\
\text { displacement }(\mathrm{mm})\end{array}$ & $\begin{array}{c}\text { Peak } \\
\text { acceleration }\left(\mathrm{mm} / \mathrm{s}^{2}\right)\end{array}$ \\
\hline 0 & 0 & 0 & 30 & 13.79 & $6.83 \mathrm{E}+05$ \\
\hline 0 & 0 & 10 & 30 & 14.07 & $6.82 \mathrm{E}+05$ \\
\hline 0 & 0 & 20 & 30 & 14.08 & $6.81 \mathrm{E}+05$ \\
\hline 0 & 0 & 0 & 45 & 19.48 & $4.62 \mathrm{E}+05$ \\
\hline 0 & 0 & 10 & 45 & 19.74 & $4.42 \mathrm{E}+05$ \\
\hline 0 & 0 & 20 & 45 & 19.76 & $4.39 \mathrm{E}+05$ \\
\hline 10 & 20 & 20 & 30 & 41.09 & $3.74 \mathrm{E}+05$ \\
\hline 20 & 10 & 20 & 30 & 49.95 & $3.47 \mathrm{E}+05$ \\
\hline 30 & 10 & 60 & 45 & 80.19 & $2.74 \mathrm{E}+05$ \\
\hline 30 & 60 & 10 & 45 & 99.77 & $2.33 \mathrm{E}+05$ \\
\hline 50 & 80 & 10 & 45 & 139.03 & $2.15 \mathrm{E}+05$ \\
\hline 150 & 150 & 70 & 45 & 162.24 & $2.00 \mathrm{E}+05$ \\
\hline 80 & 90 & 40 & 45 & 168.66 & $1.70 \mathrm{E}+05$ \\
\hline 100 & 120 & 10 & 60 & 174.00 & $1.68 \mathrm{E}+05$ \\
\hline 100 & 150 & 130 & 60 & 243.96 & $1.41 \mathrm{E}+05$ \\
\hline 60 & 150 & 0 & 60 & 321.11 & $1.23 \mathrm{E}+05$ \\
\hline 110 & 40 & 60 & 60 & 374.59 & $8.87 \mathrm{E}+04$ \\
\hline 150 & 150 & 0 & 75 & 396.89 & $7.64 \mathrm{E}+04$ \\
\hline 90 & 60 & 100 & 75 & 413.27 & $7.61 \mathrm{E}+04$ \\
\hline 150 & 100 & 140 & 90 & 421.56 & $7.17 \mathrm{E}+04$ \\
\hline 110 & 90 & 0 & 90 & 428.34 & $6.88 \mathrm{E}+04$ \\
\hline 140 & 140 & 140 & 90 & 429.61 & $6.25 \mathrm{E}+04$ \\
\hline 20 & 60 & 20 & 75 & 442.90 & $6.02 \mathrm{E}+04$ \\
\hline 70 & 0 & 100 & 90 & 443.12 & $4.84 \mathrm{E}+04$ \\
\hline 70 & 110 & 20 & 90 & 451.08 & $4.83 \mathrm{E}+04$ \\
\hline
\end{tabular}


Table 4.12.

Composite tube problem - axial impact. Optimal feasible designs

\begin{tabular}{|c|c|c|c|c|c|}
\hline $\begin{array}{c}\mathrm{x}_{1} \\
(\mathrm{~mm})\end{array}$ & $\begin{array}{c}\mathrm{x}_{2} \\
(\mathrm{~mm})\end{array}$ & $\begin{array}{c}\mathrm{x}_{3} \\
(\mathrm{~mm})\end{array}$ & $\begin{array}{c}\theta \\
\text { (deg.) }\end{array}$ & $\begin{array}{c}\text { Maximum } \\
\text { displacement }(\mathrm{mm})\end{array}$ & $\begin{array}{c}\text { Peak } \\
\text { acceleration }\left(\mathrm{mm} / \mathrm{s}^{2}\right)\end{array}$ \\
\hline 0 & 0 & 0 & 30 & 13.79 & $6.83 \mathrm{E}+05$ \\
\hline 0 & 0 & 10 & 30 & 14.07 & $6.82 \mathrm{E}+05$ \\
\hline 0 & 0 & 10 & 45 & 19.74 & $4.42 \mathrm{E}+05$ \\
\hline 0 & 0 & 20 & 45 & 19.76 & $4.39 \mathrm{E}+05$ \\
\hline 20 & 10 & 0 & 45 & 45.25 & $3.39 \mathrm{E}+05$ \\
\hline 20 & 20 & 30 & 45 & 54.23 & $3.23 \mathrm{E}+05$ \\
\hline 20 & 40 & 20 & 45 & 73.21 & $2.58 \mathrm{E}+05$ \\
\hline 20 & 60 & 130 & 45 & 88.62 & $2.34 \mathrm{E}+05$ \\
\hline 20 & 60 & 80 & 45 & 89.65 & $2.22 \mathrm{E}+05$ \\
\hline 10 & 150 & 120 & 45 & 98.33 & $2.22 \mathrm{E}+05$ \\
\hline 40 & 60 & 10 & 45 & 109.28 & $1.78 \mathrm{E}+05$ \\
\hline 20 & 130 & 80 & 45 & 118.66 & $1.67 \mathrm{E}+05$ \\
\hline 20 & 130 & 150 & 45 & 118.88 & $1.66 \mathrm{E}+05$ \\
\hline 50 & 150 & 120 & 45 & 128.57 & $1.59 \mathrm{E}+05$ \\
\hline 90 & 80 & 10 & 45 & 146.59 & $1.53 \mathrm{E}+05$ \\
\hline 80 & 80 & 10 & 45 & 166.09 & $1.32 \mathrm{E}+05$ \\
\hline
\end{tabular}




\subsubsection{Oblique Impact}

The purpose of this example is to determine the optimal design of the composite tube under non-symmetric loading. To obtain the non-symmetric impact condition, the rigid wall is inclined $30^{\circ}$ as shown in Fig. 4.25.

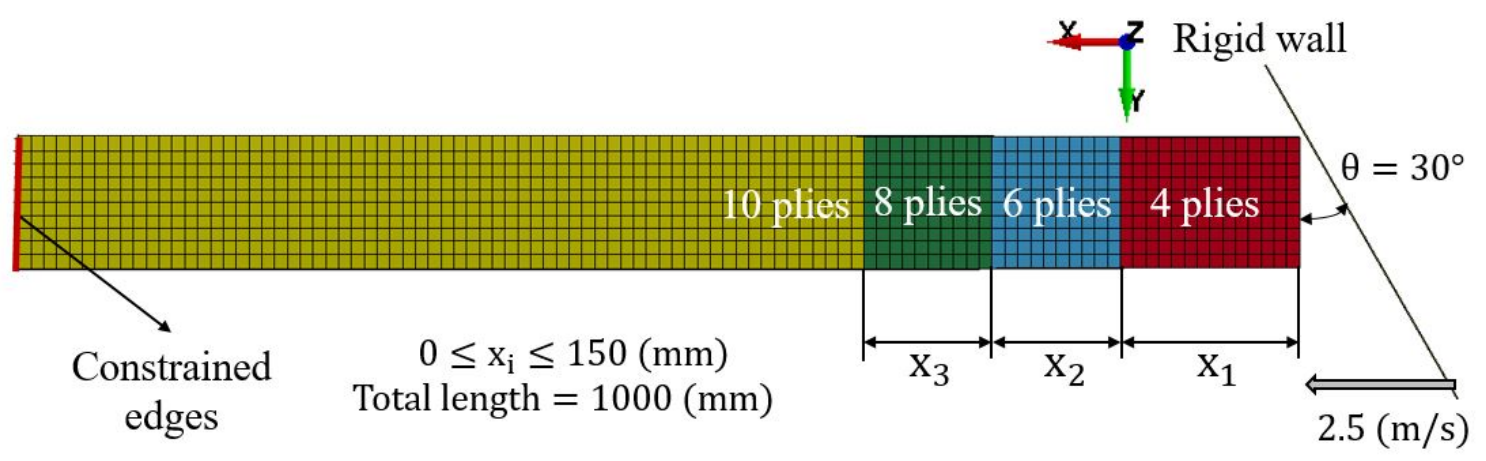

Figure 4.25. Composite tube problem - oblique impact.

The same 43 LHS samples of Table 4.8 are used to generate the Kriging surrogates to be validated. According to Table 4.13, the best surrogate for the displacement has a first-order regression function and exponential correlation $\left(\mathrm{PRESS}_{\mathrm{RMSE}}=17.8577\right.$ $\mathrm{mm}$ ). Table 4.14 suggests a zero-order regression function and exponential correlation for the acceleration metamodel $\left(\mathrm{PRESS}_{\mathrm{RMSE}}=6.20 \mathrm{E}+03 \mathrm{~mm} / \mathrm{s}^{2}\right)$. The responses of the initial samples are shown in Fig. 4.26.

Table 4.13.

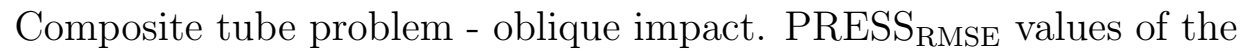
Kriging metamodels for the wall displacement.

\begin{tabular}{|ccc|ccc|}
\hline \multicolumn{2}{|c|}{ Exponential correlation } & \multicolumn{3}{c|}{ Gaussian correlation } \\
\hline regpoly0 & regpoly1 & regpoly2 & regpoly0 & regpoly1 & regpoly2 \\
22.1306 & 17.8577 & 191.5058 & 19.4353 & 20.9978 & 186.2365 \\
\hline
\end{tabular}

Fig. 4.27 contains the responses of the first four EGO iterations. Although the problem complexity, EGO expands the Pareto front in iterations two and three. 
Table 4.14.

Composite tube problem - oblique impact. PRESS $\mathrm{RMSE}$ values of the Kriging metamodels for the peak acceleration of the wall.

\begin{tabular}{|ccc|ccc|}
\hline \multicolumn{2}{|c|}{ Exponential correlation } & \multicolumn{3}{c|}{ Gaussian correlation } \\
\hline regpoly0 & regpoly1 & regpoly2 & regpoly0 & regpoly1 & regpoly2 \\
$6.20 \mathrm{E}+03$ & $7.23 \mathrm{E}+03$ & $1.75 \mathrm{E}+04$ & $7.55 \mathrm{E}+03$ & $7.18 \mathrm{E}+03$ & $1.95 \mathrm{E}+04$ \\
\hline
\end{tabular}

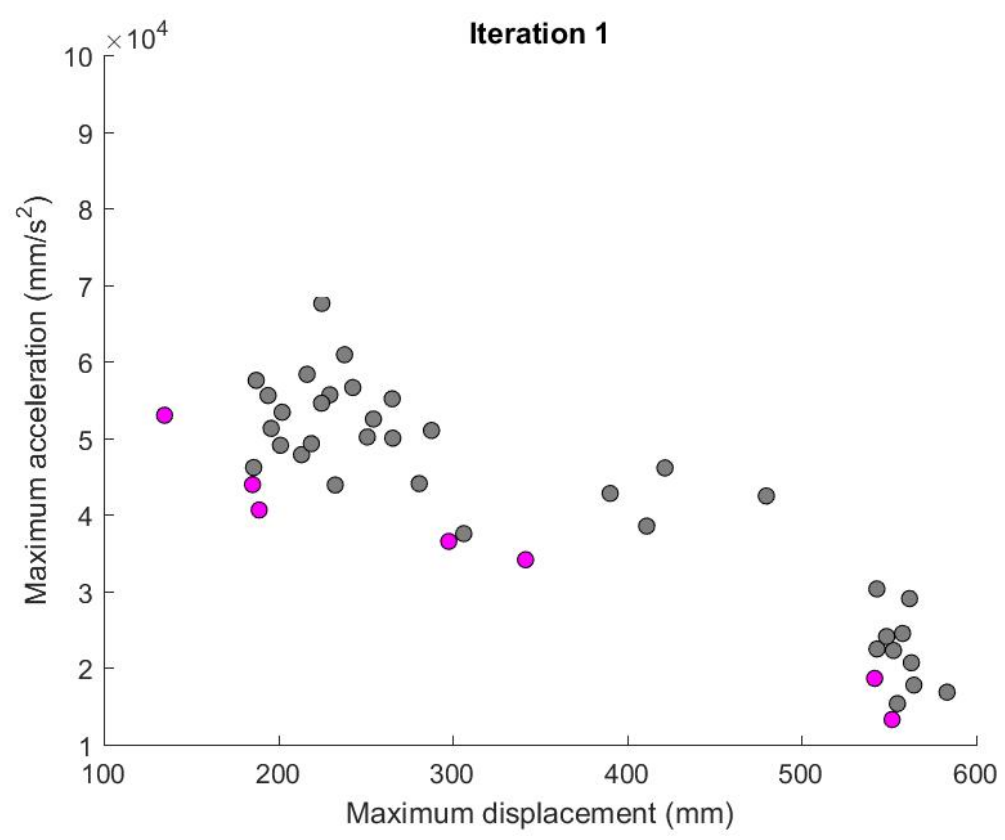

Figure 4.26. Composite tube problem - oblique impact. Initial responses using the 43 LHS samples. LHS samples (grey), Pareto front (purple).

Fig. 4.28 shows the distribution of the responses at the last iteration. As in the axial loading case, EGO tends to sample designs with large displacements and low accelerations. The designs whose displacements are larger than $235(\mathrm{~mm})$ are non-feasible because their supports fail during the impact.

Constrained EI function is implemented to avoid the sampling of non-feasible designs. The algorithm is restricted to select designs with wall displacement less than $235 \mathrm{~mm}$. The constraint effect is shown in Fig. 4.29. The constrained formulation concentrates almost all the samples in the feasible design region. At the end of 

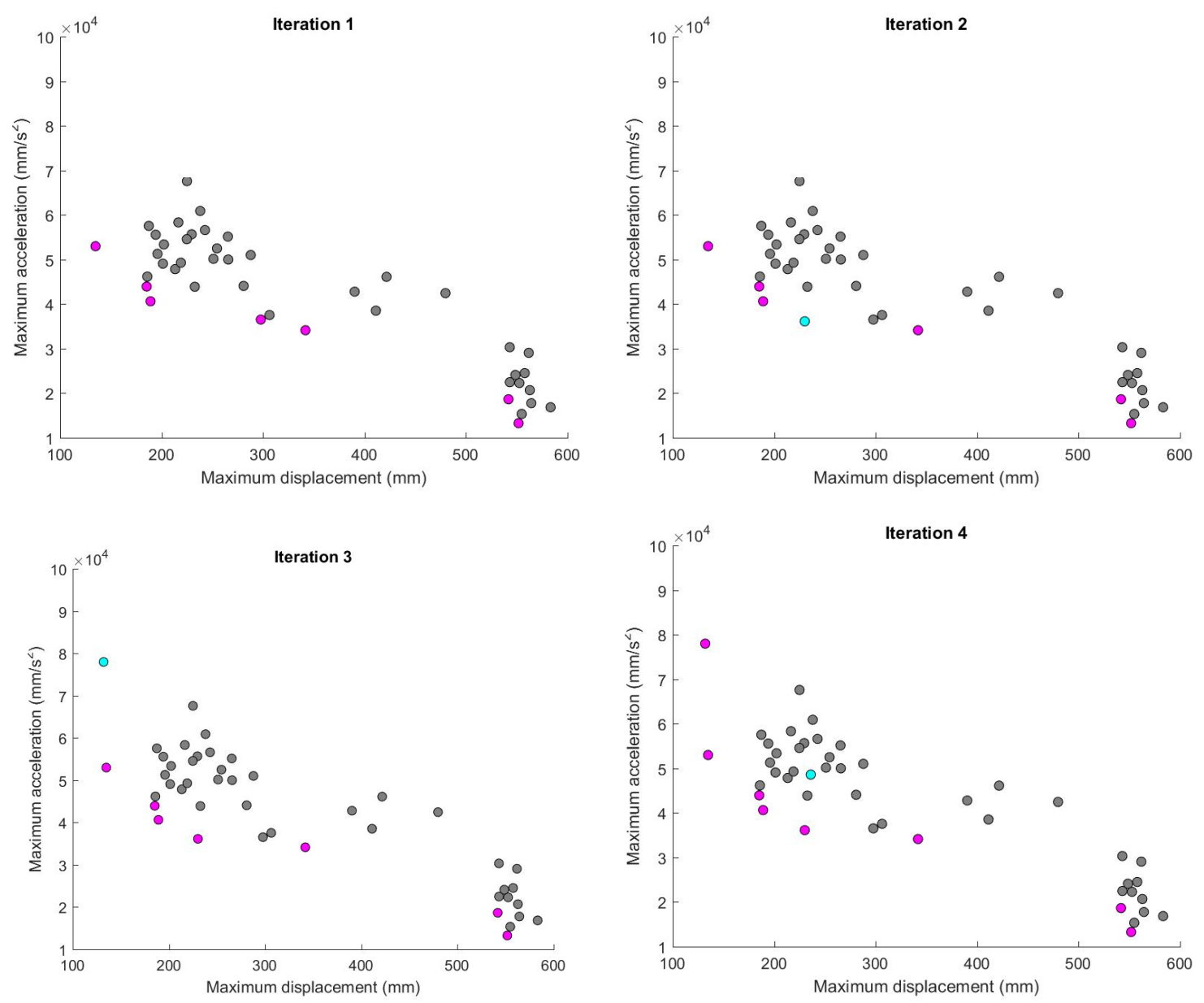

Figure 4.27. EGO optimization progress - Composite tube problem, oblique impact. Dominated designs (grey), Pareto front (purple), last sampled design (cyan). Iterations 1, 2, 3 and 4 .

the optimization, more feasible designs are present in the Pareto front. Table 4.15 contains the optimal designs using the constrained EI approach. 


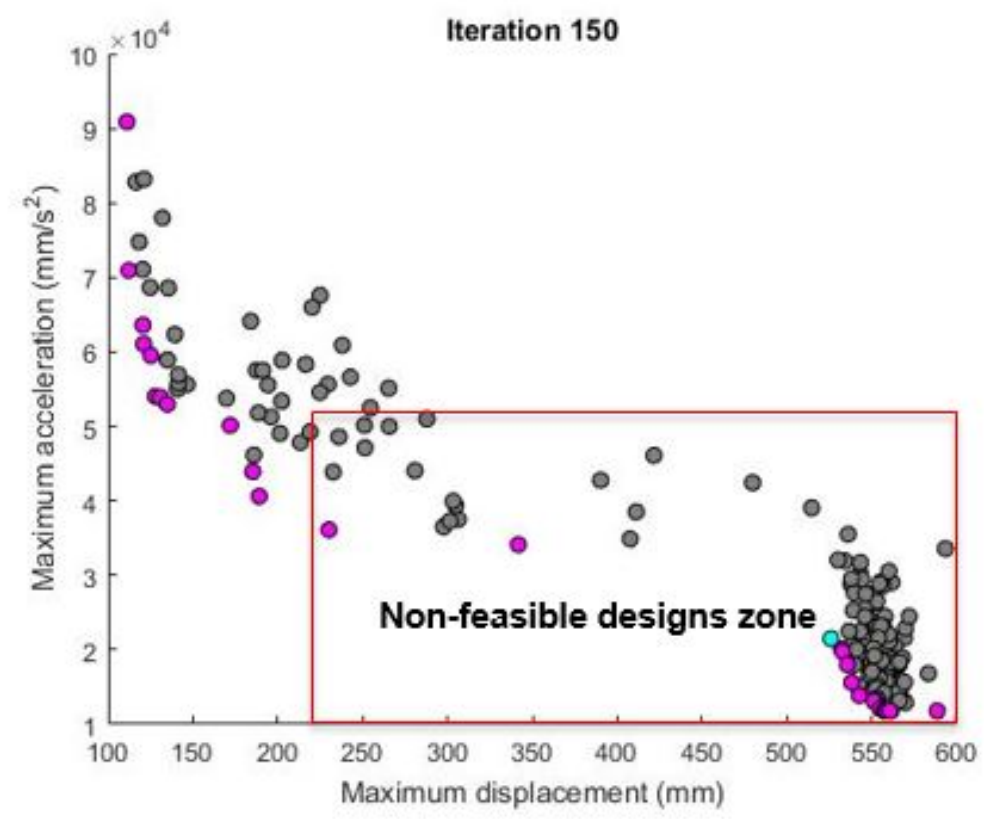

Figure 4.28. Composite tube problem - oblique impact. Non-feasible designs in the final Pareto front.

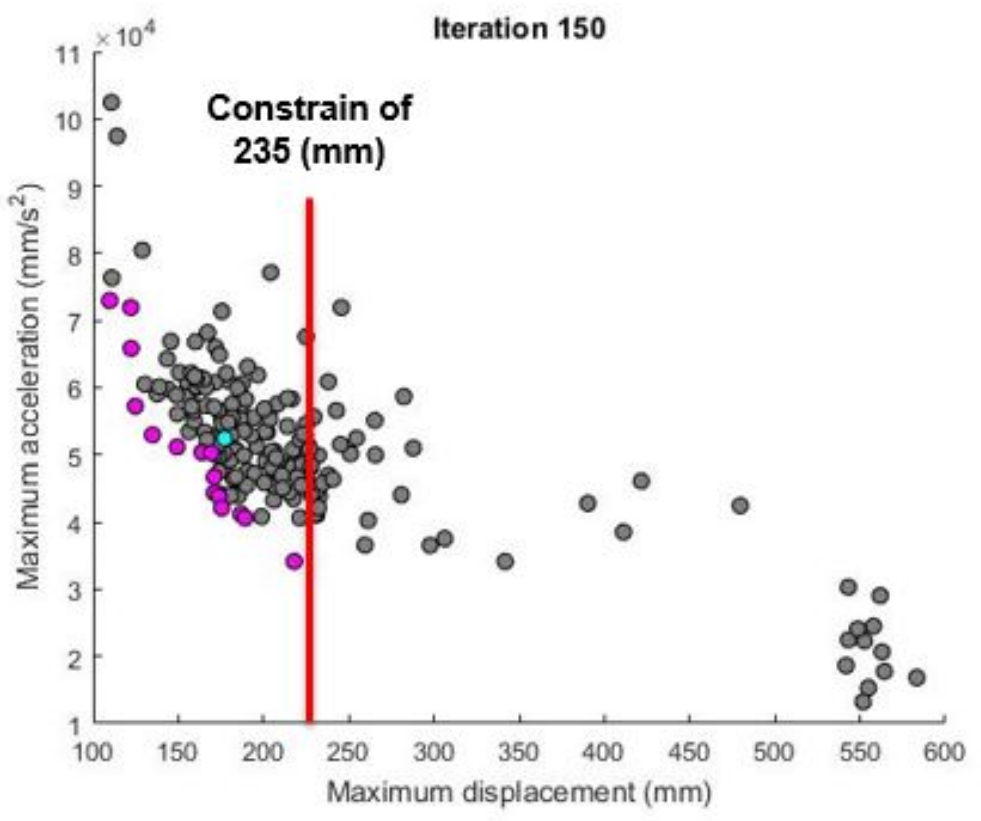

Figure 4.29. Composite tube problem - oblique impact. Responses at the last iteration using constrained improvement formulation. 
Table 4.15.

Composite tube problem - oblique impact. Optimal feasible designs

\begin{tabular}{|c|c|c|c|c|c|}
\hline $\begin{array}{c}\mathrm{x}_{1} \\
(\mathrm{~mm})\end{array}$ & $\begin{array}{c}\mathrm{x}_{2} \\
(\mathrm{~mm})\end{array}$ & $\begin{array}{c}\mathrm{x}_{3} \\
(\mathrm{~mm})\end{array}$ & $\begin{array}{c}\theta \\
\text { (deg.) }\end{array}$ & $\begin{array}{c}\text { Maximum } \\
\text { displacement }(\mathrm{mm})\end{array}$ & $\begin{array}{c}\text { Peak } \\
\text { acceleration }\left(\mathrm{mm} / \mathrm{s}^{2}\right)\end{array}$ \\
\hline 0 & 0 & 0 & 30 & 109.76 & $7.30 \mathrm{E}+04$ \\
\hline 0 & 20 & 0 & 30 & 122.13 & $7.19 \mathrm{E}+04$ \\
\hline 10 & 0 & 0 & 30 & 122.34 & $6.59 \mathrm{E}+04$ \\
\hline 10 & 0 & 10 & 30 & 124.85 & $5.72 \mathrm{E}+04$ \\
\hline 10 & 20 & 10 & 30 & 134.88 & $5.30 \mathrm{E}+04$ \\
\hline 10 & 20 & 10 & 45 & 149.33 & $5.12 \mathrm{E}+04$ \\
\hline 0 & 60 & 150 & 30 & 163.86 & $5.04 \mathrm{E}+04$ \\
\hline 10 & 30 & 100 & 45 & 169.50 & $5.03 \mathrm{E}+04$ \\
\hline 10 & 0 & 100 & 0 & 171.09 & $4.67 \mathrm{E}+04$ \\
\hline 10 & 70 & 150 & 30 & 171.10 & $4.44 \mathrm{E}+04$ \\
\hline 10 & 0 & 110 & 0 & 173.85 & $4.38 \mathrm{E}+04$ \\
\hline 0 & 120 & 140 & 30 & 175.49 & $4.21 \mathrm{E}+04$ \\
\hline 0 & 0 & 130 & 0 & 186.66 & $4.12 \mathrm{E}+04$ \\
\hline 0 & 10 & 130 & 0 & 189.10 & $4.06 \mathrm{E}+04$ \\
\hline 10 & 70 & 150 & 0 & 218.06 & $3.41 \mathrm{E}+04$ \\
\hline
\end{tabular}

Fig. 4.30 compares the constrained and unconstrained approaches. Constrained formulation leads to better results since it generates a wider expansion of the Pareto front.

The convergence of the constrained approach is illustrated in Fig. 4.31. Although the complexity of the problem, the constrained EI approaches to zero after each iteration but more evaluations are needed to satisfy the stopping criteria. 


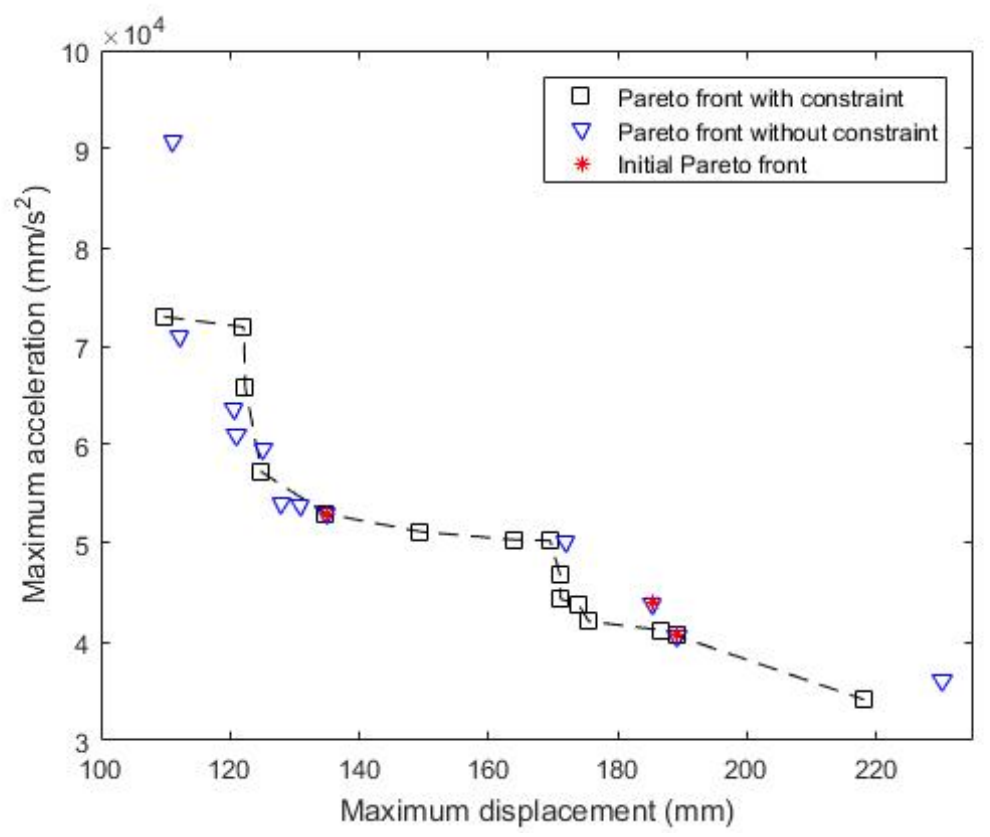

Figure 4.30. Composite tube problem - oblique impact. Comparison of Pareto fronts using constrained and unconstrained expected improvement.

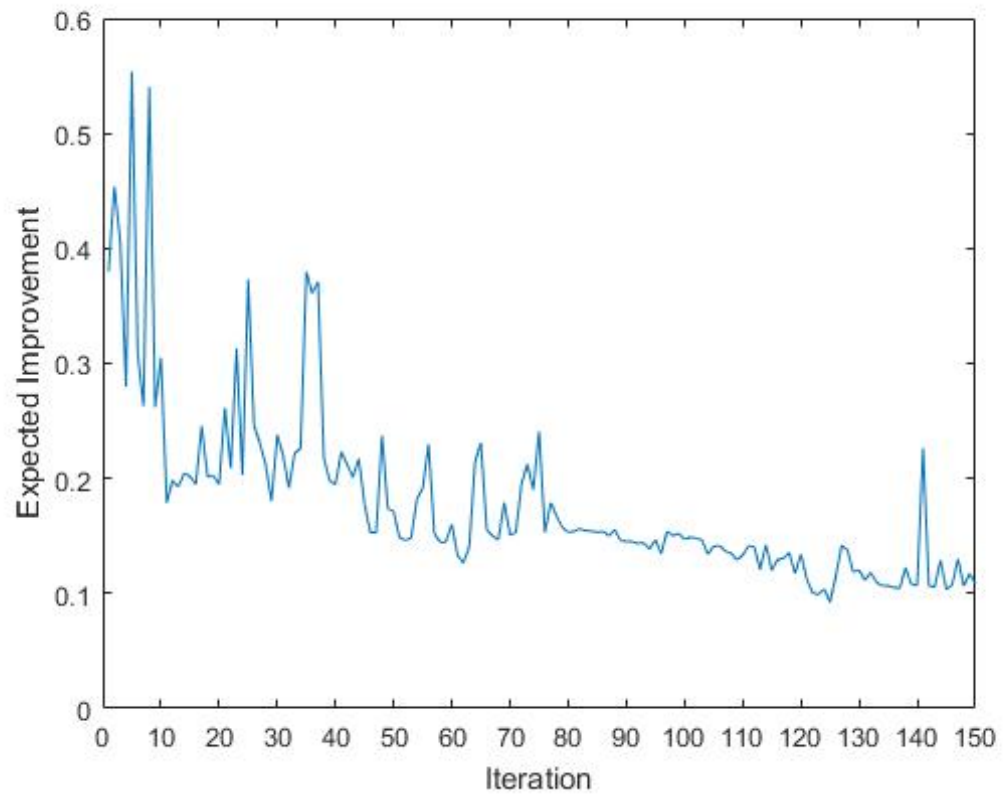

Figure 4.31. Composite tube problem - oblique impact. Expected improvement at each iteration. 


\section{SUMMARY AND RECOMMENDATIONS}

Nowadays, composites material structures are widely used in fields such as Aerospace, Automobile, Construction and Energy Industry. Correctly designed, composite materials provide many benefits like weight reduction, durability, high strength and energy absorption. Their performance depends on different variables such as the matrix and fiber materials, stacking sequence, ply thickness, and geometry. Optimization design techniques are used to determine the optimal parameters.

The implementation of optimization methods is straightforward when dealing with simple problems. However, complications arise in the presence of impact events such as the examples included in this work. The use of explicit finite formulations is necessary to study dynamic models due to the presence of multi-body contact, and nonlinearities in the material and geometry of the parts. This study has explored the options provided by the FEA code LS-DYNA. A detailed explanation of MAT_054/055 (Enhanced Composite Damage Model) is included in Chapter 2. MAT_054/055 is widely used due to its ease of implementation and ability to capture the structural behavior of composite parts. Those features were corroborated with the simulations performed in this study. For the optimization of composite parts under dynamic loading, the implementation of gradient-based optimization techniques and population-based methods is impractical due to the complexity and long-running time requirements of the FEA simulations. Therefore, surrogate-based global optimization approach was applied in this work. Latin hypercube sampling, Kriging metamodel, cross-validation and Expected Improvement (EI) function were used to implement the Efficient Global Optimization (EGO) algorithm.

Different application examples are included in this work. The armor plate problems illustrate how the EI function directs the optimization towards the global optimum while the surrogate is enhanced after each iteration. The composite tube 
problems show that the collapse of composite parts can be controlled by an optimal distribution of the number of plies along the part and the correct fiber orientation. Due to the brittle behavior of composite materials, non-feasible designs were encountered in the final Pareto front. A constrained formulation of the EI function was necessary to guarantee the feasibility of the designs selected by EGO. The numerical examples show that the composite parts can be designed to resist impact and to absorb energy during crash events.

EGO uses the uncertainties in the predictions of the metamodel to direct the searching process but it does not include uncertainties in the design variables or in the parameters of the model. The design of composite parts can be extended to the reliable and robust optimization field.

Kriging surrogates present more mathematical models than interpolation. Information from high fidelity sources can be combined with low fidelity data. In addition, Kriging metamodels can be used as an approximation technique that is suitable when dealing with noisy information. These are potential features for the optimization of composite parts.

One of the limitations faced in this study is the difficulty to modify the FE mesh in the LS-DYNA input file. Therefore, the geometry of the composite part could not be changed. If an external mesher is interfaced with LS-DYNA, EGO can be used to optimize the shape of composite parts.

FEA simulations of composites are highly costly. Parallel computing can reduce the optimization time by enabling the evaluation of multiple samples at the same time. For example, multiple processors can simulate the initial samples required by EGO since they are independent. Additionally, EGO can be combined with another optimization technique, e.g., Genetic Algorithms (GA). GA can propose other designs for metamodel enhancing. 
REFERENCES 


\section{REFERENCES}

[1] C.-K. Park, C.-D. S. Kan, W. T. Hollowell, and S. I. Hill, "Investigation of opportunities for lightweight vehicles using advanced plastics and composites," National Highway Traffic Safety Administration, Tech. Rep., pp. 4-17, 2012.

[2] G. Mastinu, M. Gobbi, and C. Miano, Optimal design of complex mechanical systems: with applications to vehicle engineering. Springer Science \& Business Media, pp. 1-12, 2007.

[3] R. Kathiravan and R. Ganguli, "Strength design of composite beam using gradient and particle swarm optimization," Composite Structures, vol. 81, no. 4, pp. 471-479, 2007.

[4] S. Rao, Engineering optimization: theory and practice. John Wiley \& Sons, pp. 335-370, 2009.

[5] E. Lund, "Buckling topology optimization of laminated multi-material composite shell structures," Composite Structures, vol. 91, no. 2, pp. 158-167, 2009.

[6] M. Luersen and R. Lopez, "A genetic algorithm for optimization of hybrid laminated composite plates," in Optimization of Structures and Components. Springer, 2013, pp. 49-71.

[7] F. Almeida and A. Awruch, "Design optimization of composite laminated structures using genetic algorithms and finite element analysis," Composite structures, vol. 88, no. 3, pp. 443-454, 2009.

[8] A. Muc and W. Gurba, "Genetic algorithms and finite element analysis in optimization of composite structures," Composite Structures, vol. 54, no. 2, pp. 275-281, 2001.

[9] S. Omkar, J. Senthilnath, R. Khandelwal, G. N. Naik, and S. Gopalakrishnan, "Artificial bee colony (ABC) for multi-objective design optimization of composite structures," Applied Soft Computing, vol. 11, no. 1, pp. 489-499, 2011.

[10] H. Ghiasi, K. Fayazbakhsh, D. Pasini, and L. Lessard, "Optimum stacking sequence design of composite materials part II: Variable stiffness design," Composite Structures, vol. 93, no. 1, pp. 1-13, 2010.

[11] J. Carruthers, A. Kettle, and A. Robinson, "Energy absorption capability and crashworthiness of composite material structures: a review," Applied Mechanics Reviews, vol. 51, no. 10, pp. 635-649, 1998.

[12] G. L. Farley and R. M. Jones, "Crushing characteristics of continuous fiberreinforced composite tubes," Journal of composite Materials, vol. 26, no. 1, pp. 37-50, 1992. 
[13] H. Fang, M. Rais-Rohani, Z. Liu, and M. Horstemeyer, "A comparative study of metamodeling methods for multiobjective crashworthiness optimization," Computers and Structures, vol. 83, no. 25, pp. 2121-2136, 2005.

[14] X. Gu, G. Sun, G. Li, L. Mao, and Q. Li, "A comparative study on multiobjective reliable and robust optimization for crashworthiness design of vehicle structure," Structural and Multidisciplinary Optimization, vol. 48, no. 3, pp. 669-684, 2013.

[15] A. Forrester, A. Sobester, and A. Keane, Engineering design via surrogate modelling: a practical guide. John Wiley \& Sons, pp. 179-186, 2008.

[16] D. R. Jones, M. Schonlau, and W. J. Welch, "Efficient global optimization of expensive black-box functions," Journal of Global optimization, vol. 13, no. 4, pp. 455-492, 1998.

[17] B. Wade, "Capturing the energy absorbing mechanisms of composite structures under crash loading," Ph.D. dissertation, University of Washington, p.p 22-23, 2014.

[18] E. Moncayo, H. Wagner, and K. Drechsler, "Benchmarks for composite delamination using LS-DYNA 971: Low velocity impact," DYNAmore GmbH, pp. 13-22, 2007.

[19] M. Chatiri, T. Güll, and A. Matzenmiller, "An assessment of the new LS-DYNA layered solid element: basics, patch simulation and its potential for thick composite structure analysis," in 7th European LS-DYNA Conference, Salzburg, pp. 1-12, 2009.

[20] K. Schweizerhof, K. Weimar, T. Munz, and T. Rottner, "Crashworthiness analysis with enhanced composite material models in LS-DYNA-merits and limits," in LS-DYNA World Conference, Detroit, MI, pp. 1-17, 1998.

[21] "LS-DYNA keyword users manual: Material models," Livermore Software Technology Corporation, vol. 2, pp. 281-291, 2013.

[22] P. Feraboli and M. Rassaian, "Proceedings of the CMH-17 (MIL-HDBK-17) crashworthiness working group numerical round robin," Costa Mesa, pp. 1-25, 2010.

[23] M. Osborne, "Single-element characterization of the LS-DYNA MAT54 material model," Ph.D. dissertation, University of Washington, pp. 24-28, 2012.

[24] B. Wade, P. Feraboli, M. Osborne, and M. Rassaian, "Simulating laminated composite materials using LS-DYNA material model MAT54: Single-element investigation. Final report," in U.S. Department of Transportation, Federal Aviation Administration, pp. 1-7, 2015.

[25] J. O. Hallquist et al., "LS-DYNA keyword users manual," Livermore Software Technology Corporation, vol. 970, pp. 1-2, 2007.

[26] M. Andersson and P. Liedberg, "Crash behavior of composite structures," A CAE benchmarking study, Master's thesis in Applied Mechanics. Chalmers University of Technology. Göteborg, Sweden, pp. 21-24, 2014. 
[27] K. Liu, A. Tovar, E. Nutwell, and D. Detwiler, "Thin-walled compliant mechanism component design assisted by machine learning and multiple surrogates," SAE Technical Paper, Tech. Rep., pp. 1-12, 2015.

[28] M. Costas, J. Díaz, L. Romera, and S. Hernández, "A multi-objective surrogatebased optimization of the crashworthiness of a hybrid impact absorber," International Journal of Mechanical Sciences, vol. 88, pp. 46-54, 2014.

[29] H. B. Nielsen, S. N. Lophaven, and J. Sondergaard, "DACE, a MATLAB Kriging toolbox," Informatics and mathematical modelling. Lyngby-Denmark: Technical University of Denmark, pp. 1-24, 2002.

[30] I. Couckuyt, T. Dhaene, and P. Demeester, "ooDACE toolbox, A Matlab Kriging toolbox: getting started," Universiteit Gent, pp. 3-15, 2013.

[31] R. H. Myers, D. C. Montgomery, and C. M. Anderson-Cook, Response surface methodology: process and product optimization using designed experiments. John Wiley \& Sons, pp. 1-12, 2016.

[32] M. D. McKay, R. J. Beckman, and W. J. Conover, "A comparison of three methods for selecting values of input variables in the analysis of output from a computer code," Technometrics, vol. 42, no. 1, pp. 55-61, 2000.

[33] M. E. Johnson, L. M. Moore, and D. Ylvisaker, "Minimax and maximin distance designs," Journal of statistical planning and inference, vol. 26, no. 2, pp. 131-148, 1990.

[34] A. B. Owen, "Controlling correlations in latin hypercube samples," Journal of the American Statistical Association, vol. 89, no. 428, pp. 1517-1522, 1994.

[35] F. A. Viana, R. T. Haftka, and V. Steffen, "Multiple surrogates: how crossvalidation errors can help us to obtain the best predictor," Structural and Multidisciplinary Optimization, vol. 39, no. 4, pp. 439-457, 2009.

[36] C. López, O. Bacarreza, A. Baldomir, S. Hernández, and M. F. Aliabadi, "Reliability-based design optimization of composite stiffened panels in postbuckling regime," Structural and Multidisciplinary Optimization, pp. 1-21, 2016.

[37] J. Tomblin, J. Sherraden, W. Seneviratne, and K. Raju, "A-basis and B-basis design allowables for epoxy-based prepreg: Toray T700GC-12k-31E/2510 unidirectional tape," in NASA AGATE Program, pp. 1-2, 2002.

[38] B. Wade, P. Feraboli, and M. Osborne, "Simulating laminated composites using LS-DYNA material model MAT54 part I:[0] and [90] ply single-element investigation," in FAA Joint Advanced Materials and Structures (JAMS) Centre of Excellence, pp. 1-2, 2012. 